\title{
3. TEPHRAS OF THE IZU-BONIN FOREARC (SITES 787, 792, AND 793) ${ }^{1}$
}

\author{
Kantaro Fujioka, ${ }^{2}$ Yoshiko Matsuo, ${ }^{3}$ Akira Nishimura, ${ }^{4}$ Masato Koyama, ${ }^{3}$ and Kelvin S. Rodolfo ${ }^{5}$
}

\begin{abstract}
Numerous marine tephra layers cored at Sites 792 and 793 in the Izu-Bonin forearc region offer additional information about the timing and spatial characteristics of arc volcanism and the evolution of island arcs. Explosive volcanism along the Izu-Bonin Arc, with maxima just before rifting of the arc at $\sim 40$ and 5- $0 \mathrm{Ma}$, produced black and white tephras of variable grain sizes and chemical compositions. Most of the tephras belong chemically to low-K and low-alkali tholeiitic rock series with a few tephra of the high-K and alkalic rock series. Most of the tephras (low-K series) were derived from the Izu-Bonin Arc, although a few were produced far to the west of the Izu-Bonin Arc (e.g., from the Ryukyu Arc). Black tephras may have come from nearby sources, such as Aogashima, Sumisu, and Torishima islands. The high-K series of tephras, within the sediments younger than $3 \mathrm{Ma}$, may reflect thickening of the island-arc crust.
\end{abstract}

\section{INTRODUCTION}

Studies of marine tephra of the Izu-Bonin Arc (Figs. 1 and 2) have lagged behind those of other regions because virtually the entire area is submerged, and the islands are so small that it is difficult to correlate marine tephras to their land sources (Machida and Arai, 1983, 1988; Furuta et al., 1986). Before the marine surveys began, geologic work along the volcanic front of the Izu-Bonin Arc emphasized detailed mapping of the younger volcanic islands of the Izu-Bonin Arc (Oshima, Toshima, Miyakejima, Hachijojima, and Myojinsho; Nakamura, 1964; Isshiki, 1959, 1960, 1978, 1980, 1984; Niino et al., 1953) and older basaltic rocks of the Bonin Islands, as well as the rhyolites on Kozushima, Shikinejima, and Niijima islands, which obliquely cross the Izu-Bonin volcanic front (Shiraki et al., 1978; Isshiki, 1982, 1987).

The bathymetry of the Izu-Bonin region has been mapped during surveys by the Geological Survey of Japan on the cruise ship HakureiMaru since 1974. Karig and Moore (1975a, 1975b) summarized the geology and geophysics of the Mariana and Izu-Bonin arcs from the results of Deep Sea Drilling Project (DSDP) Leg 31. A geologic map of the Izu-Bonin Arc at a scale of 1:1,000,000 was published by the Geological Survey of Japan (Honza et al., 1982). More recently, Honza and Tamaki (1985) and Yuasa and Murakami (1985) compiled the geology and geophysics of the Izu-Bonin Arc using all the data available at that time.

Nakamura (1964) performed excellent tephrochronological work on Oshima volcano; however, marine tephrochronological works of the Izu-Bonin Arc are scarce because of the lack of good piston cores, and because all previous DSDP/IPOD (International Phase of Ocean Drilling) sites along the arc were limited to the transect across the Mariana Arc during IPOD Legs 59 and 60. From these studies, volcanism in the Philippine Sea area has been placed in the context of the opening of the Shikoku and Parece Vela basins and marginal seas (Kobayashi and Nakada, 1978; Seno and Maruyama, 1984). However, drilling in the Izu-Bonin Arc did not begin until Ocean Drilling Program (ODP) Leg 126 in 1989.

\footnotetext{
'Taylor, B., Fujioka, K., et al., 1992. Proc. ODP, Sci. Results, 126: College Station, TX (Ocean Drilling Program).

${ }^{2}$ Ocean Research Institute, University of Tokyo, 1-15-1 Minamidai, Nakano, Tokyo 164, Japan (present address: Japan Marine Science and Technology Center, 2-14 Natsushima, Yokosuka, Kanagowa 238, Japan).

${ }^{3}$ Institute of Geosciences, Shizuoka University, 836 Oya, Shizuoka 422, Japan.

${ }^{4}$ Marine Geology Department, Geological Survey of Japan, 1-1-3 Higashi, Tsukuba, Ibaraki 305, Japan.

${ }^{5}$ Department of Geological Sciences, M/C 186, University of Illinois at Chicago, P.O. Box 4348, Chicago, II 60680, U.S.A.
}

After Karig (1971a, 1971b) initially demonstrated that the Philippine Sea had formed by backarc spreading, an intense debate arose about the temporal relationship between arc volcanism and backarc spreading. The volcanic activity of the Mariana Arc was documented during DSDP/IPOD Legs 31 (Karig, Ingle, et al., 1975), 58 (Klein and Kobayashi, 1980), 59 (Scott and Kroenke, 1980; Rodolfo, 1980), and 60 (Hussong and Uyeda, 1981). Karig, Ingle, et al. (1975) concluded from the results of Leg 31 that backarc spreading occurred during maxima in arc volcanism; and Karig (1983) restated and defended this position. In contrast, Scott and Kroenke (1980), Rodolfo (1980), Crawford et al. (1981), Scott et al. (1980), and Sharaskin et al. (1981) interpreted the successions drilled during Leg 59 as evidence that backarc opening behind the Mariana Arc occurred during periods of relative quiescence in arc volcanism. Discussing the results of Leg 60, Hussong and Uyeda (1981) suggested that backarc opening had no temporal relationship with arc volcanism. The results of Leg 126 bear importantly on this question.

During Leg 126, three sites were drilled in the forearc basin of the Izu-Bonin arc-trench system (Figs. 1 and 2): Sites 787 in the Aogashima Canyon, Site 792 at a fork of this canyon, and Site 793 in the middle of the upper slope basin. Oligocene to Holocene sediments and rocks of similar types (gravity-flow deposits, hemipelagites, and volcaniclastic rocks in stratigraphic ascending order) were recovered from the three sites (Fig. 3; Taylor, Fujioka, et al., 1990). The sedimentation rates for the same rock types at each of the three sites, based on biostratigraphic and magnetostratigraphic age controls (Leg 126 Shipboard Scientific Party, 1989; Taylor, Fujioka, et al., 1990), are also similar to each other: fast in the gravity-flow deposits, very slow for hemipelagic rocks, and intermediate for volcaniclastic rocks. More than 300 tephra layers were recovered, from APC cores in the shallower sediment sections of these three sites and from rotary cores ( $\mathrm{RCB}$ ) taken from deeper, sedimentary rock portions. During ODP Leg 125, five other sites were also drilled in the Izu-Bonin forearc (Leg 125 Shipboard Scientific Party, 1989). Two of these sites (782 and 786) reached the Eocene to Oligocene volcanic basement of the outer-arc high, and 116 tephra layers were recovered that offer important information about the history of both explosive and effusive volcanism that took place on the outer-arc high during the Tertiary (Leg 125 Shipboard Scientific Party, 1989).

We present here shipboard descriptions and microscopic characteristics of the tephra layers obtained during Leg 126, and the results of laboratory data on grain-size and chemical analyses conducted on tephras. We discuss the nature and origin of the marine tephras around the Izu-Bonin forearc as inferred from this information, which was determined at the Ocean Research Institute of the University of Tokyo. Site 792 continuously cored Oligocene sedimentary successions with good recovery from Holes 792A, 792B, and 792E; thus, 


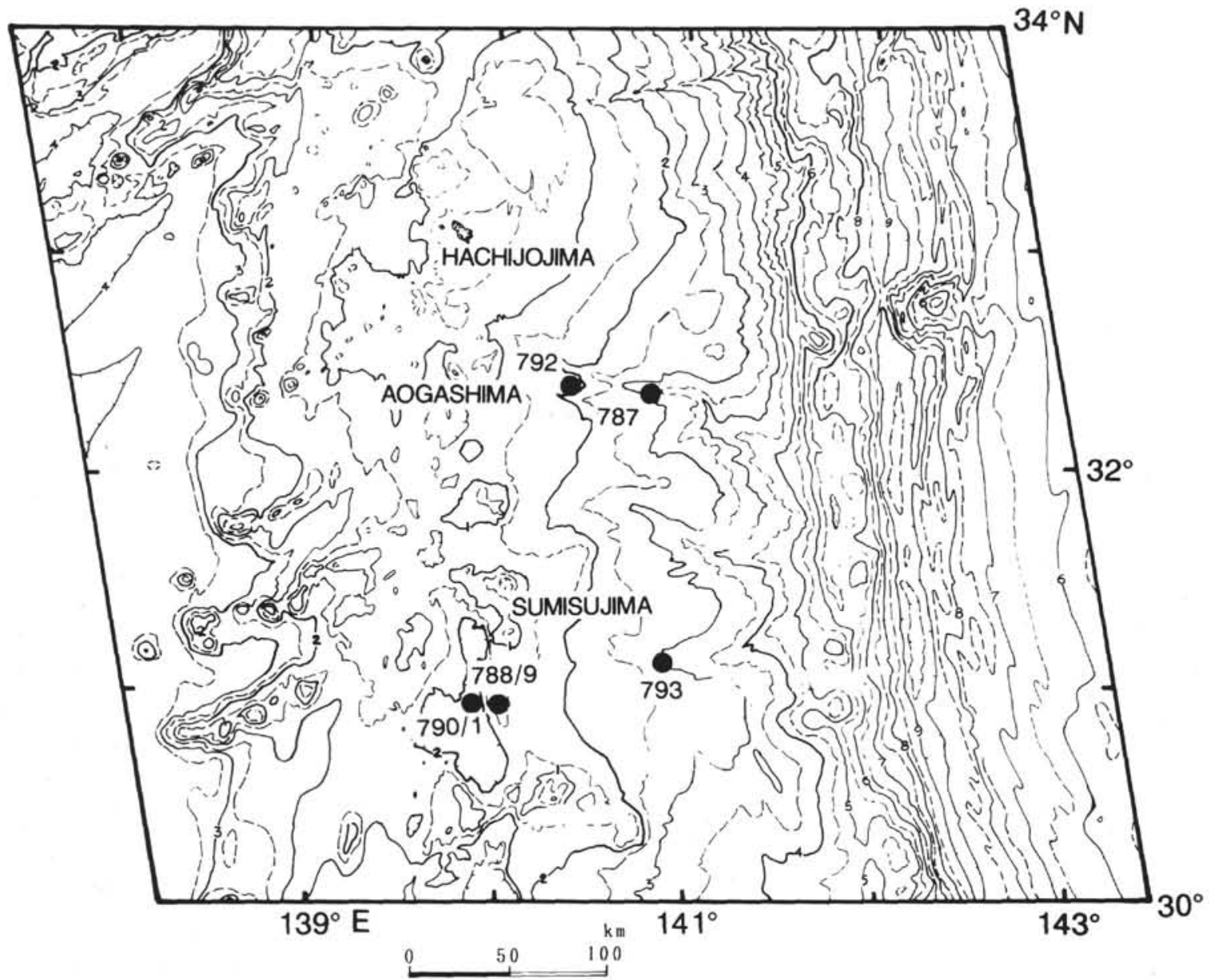

Figure 1. Bathymetric map of the Izu-Bonin arc-trench system between $30^{\circ}$ and $34^{\circ} \mathrm{N}$ and drilling sites of Legs 125 and 126 . Contour interval $=500 \mathrm{~m}$ (numbers on contour lines indicate depth in kilometers). Locations of the drill sites are indicated by filled circles.

Site 792 serves as our reference site for the Izu-Bonin forearc (Tables 1 and 2).

\section{METHODS}

Shipboard descriptions of the tephra layers (Taylor, Fujioka, et al., 1990) are used here (Tables 1 and 2). The mineral assemblages, morphologies of the glass shards, and characters of the lithic fragments in each tephra layer were determined from smear slides both at sea and onshore at the Ocean Research Institute, University of Tokyo, using standard ODP techniques (Taylor, Fujioka, et al., 1990).

Grain-size distributions for 64 tephra layers were determined by using standard sieving methods for grains $>500 \mu \mathrm{m}$, and a Shimadzu Co, Ltd., Model SALD-1100 laserbeam particle-size analyzer for grains $44-500 \mu \mathrm{m}$ in diameter. These results are presented in Appendix A (in back-pocket microfiche). The finer fractions $<44 \mu \mathrm{m}$ were first removed by suspension in distilled water; the samples were then agitated in an ultrasonic cleaner and decanted. The accuracy of grain-size distributions using this instrument is $>95 \%$.

Refractive indices of glass shards from the tephra layers were measured with the Refractive Index Measurement System, Model RIMS-86 of Kyoto Fission Track Co., Ltd., using the experimental procedures of Yokoyama et al. (1986). This refractometer is useful for the characterization of glass shards in tephras because the refractive indices of many glass shards can be determined with ease even if they are present in small amounts and are mixed with mineral and pumice grains. The accuracy of the refractive index for a single shard is $>99 \%$. Table 3 summarizes the rock types, thicknesses, grain sizes, glass/crystal/lithic ratios, constituent minerals, glass shard morphologies, and refractive indices for 116 of the tephra layers, those that we think represent primary ash falls. Reworked tephra layers are not included.

Concentrations of 12 major elements were determined by electron microprobe analyses for glass shards and mineral grains using methods similar to those described by Smith and Westgate (1969). Tephra samples were suspended in distilled water and then agitated in an ultrasonic cleaner. The clay fractions $(<2-\mu \mathrm{m}$ fraction) were removed by decanting the supernatant suspension. After drying, hand-picked shards were impregnated with epoxy resin in 5-mm holes punched in acrylic plates. After polishing, these mounts were analyzed with a JEOL Model JCXA-733 electron probe microanalyzer, with a $10-\mu \mathrm{m}$ diameter defocused beam at $15 \mathrm{kV}$ and $1.2 \times 10^{-7} \AA$, using 10 -s counting times for every oxide. The results of these analyses are listed in Appendix B (in back-pocket microfiche). Albite, corundum, quartz, periclase, adularia, hematite, Ni-olivine, wollastonite, ilmenite, Mnolivine $\mathrm{V}_{2} \mathrm{O}_{3}$, and spinel were used as standards for each oxide. The accuracy of the values for the different oxides range from $90 \%$ to $98 \%$. Other experimental procedures and conditions used in the volcanic glass analyses are discussed in detail by Fujioka et al. (1980) and Furuta and Arai (1980a, 1980b).

\section{VISUAL ASPECTS OF TEPHRA LAYERS}

Colors of the tephras are white, gray, green, and black, depending on the chemistry and the presence of lithic fragments and minerals 


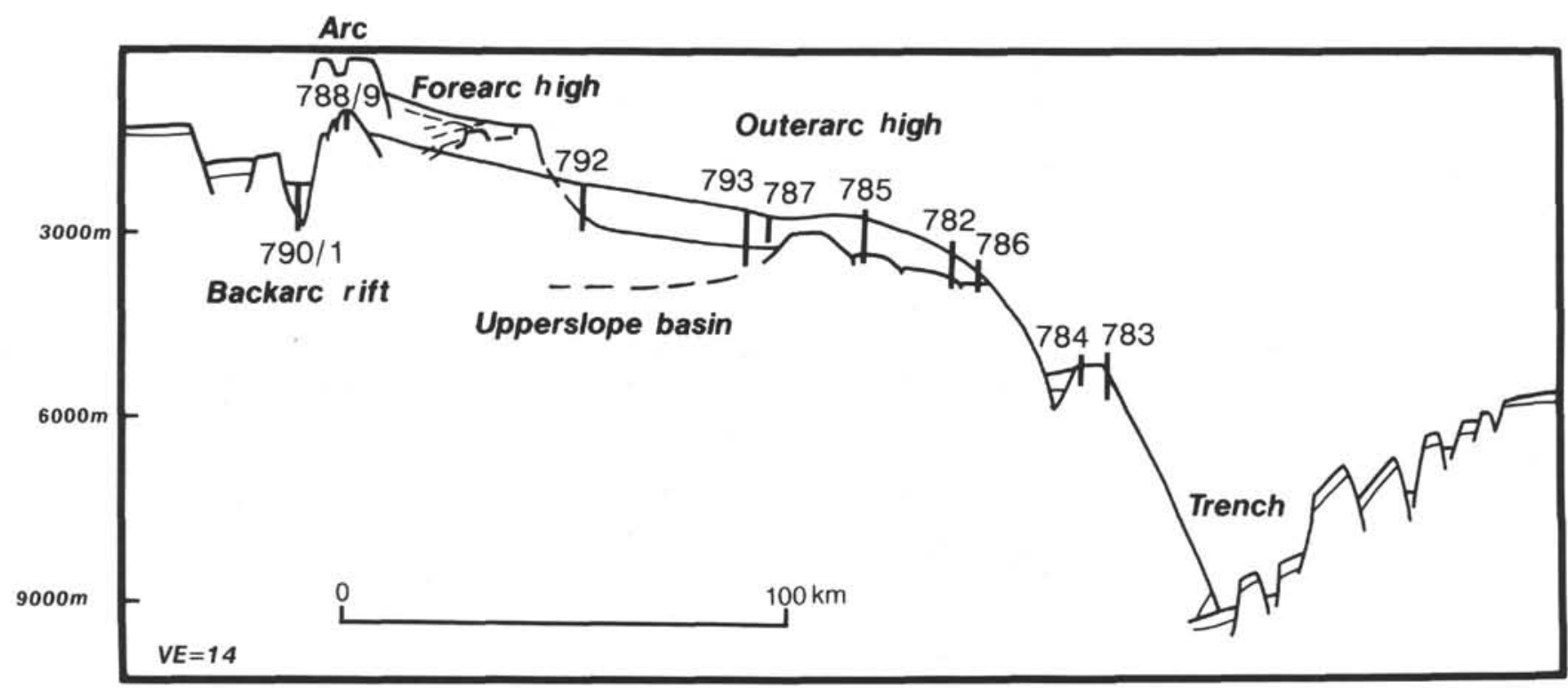

Figure 2. Schematic cross section of the Izu-Bonin arc-trench system, showing the locations of the drilling sites of Legs 125 and 126 . VE = vertical exaggeration.

(Tables 1 and 2). Glass shards are typically silt size, whereas scoria and pumice grains are sand and granule size. The tephra layers range in thickness from several millimeters to several decimeters (maximum $=146 \mathrm{~cm})$, reflecting on their distance from source areas and degrees of postdepositional bioturbation (Walker, 1971). Most of the tephra layers (Fig. 4) were $<10 \mathrm{~cm}$ thick. Beds greater than $60 \mathrm{~cm}$ thick commonly contain scoria and pumice, possibly resulting from mixing and resedimentation by turbidites. Some tephra layers showed distinct size grading, some have mixed assemblages of rhyolitic and basaltic compositions, and some display resedimented structures. Pronounced structures caused by bioturbation of bottom-dwelling organisms such as zoophycos, chondrites, and planolites were rare (Taylor, Fujioka, et al., 1990).

The tephras occur in definite layers (L; Fig. 4), small pods or pockets $(\mathrm{P})$, or dispersed in the muddy matrix (D). These occurrences had previously been observed in cores taken during IPOD Leg 57 in the Japan Trench forearc area (Fujioka et al., 1980). Each layer may be either a single bed (Fig. 4A) or a set of tephra beds with sharp contacts and no intervening mud (Figs. 4B, 4C, and 4D), thereby documenting explosive eruptions that followed each other closely in time. Both simple and multiple tephras may be a single color or mottled black and white beds. Two types of dark colored tephra layers were common; one consisted of black, coarse scoria, and the other of black, fine, well-sorted ash. Two types of pale-colored tephra layers are also common: white pumiceous debris and fine white ash.

\section{Smear Slide and Binocular Observations}

In smear slides, the volcanic glass shards are translucent-colorless, black to brown, and mixed (see "Comments" column, Table 3). Layers dominated by the translucent shards typically are composed of more than $85 \%$ glass shards with few lithic fragments. The beds with black to brown shards also have minor amounts of lithic fragments and $15 \%-40 \%$ crystal content in addition to black and brown glass shards. The mixed type contains black and translucent volcanic glass shards. Shard morphologies are given in the "Glass Shape" column of Table 3. The colorless fragments are typically bubble-wall (bw) fragments, tubular (t), or pumiceous (pum). Brown bubble-wall shards are denoted as "brbw"; black ashes normally are crystal-rich (cry). Representative morphologies are shown in the stereographic photomicrographs of Figure 5 and in the smear-slide photomicrographs of Figure 6. Plagioclase, clinopyroxenes, and opaque minerals are common in all the types of tephra layers; orthopyroxene and biotite are rare. Rare hornblendes occur in the white tephra layers and crystal-rich black ashes.

\section{FREQUENCY OF THE TEPHRA LAYERS}

The frequency of volcanic tephra layer occurrences was determined by using biostratigraphic-controlled ages from Taylor, Fujioka, et al. (1990). Site 792, with continuous recovery through the Oligocene, is our reference site for the Izu-Bonin forearc. In Figure 7, frequencies of the Oligocene tephra beds are not included because these deposits were predominantly thick, resedimented, volcaniclastic deposits, and discrete tephra layers were difficult to identify (Taylor, Fujioka, et al., 1990); however, the scientific party of Leg 125 counted the ash frequencies at Sites 782 and 786 (Tables 4 and 5 ), which were drilled on the outer-arc basement high southeast of Site 792 (Fig. 1). The ash-layer frequencies display a maximum in late Eocene to early Oligocene time (Leg 125 Shipboard Scientific Party, 1989).

Several lacunas occur in the sedimentary succession recovered from Site 792 at 1-2.2, 3.5-6, 8-9, and 13-19 Ma (Taylor, Fujioka, et al., 1990), which must be considered in analyzing the frequency. Figure 7 illustrates the frequency of tephra-layer occurrences in the successions of uppermost Oligocene to Pleistocene rocks and sediments at Site 792, our reference site. There appear to be minor peaks in tephra-layer frequencies in the middle and upper Miocene (10-11 and 6.7-6.8 Ma, respectively), and in the lower part of the upper Pliocene succession (4.5-2 Ma). In Figure 8, a similar frequency diagram for the tephra layers of Quaternary age shows two peaks: one from 1.0 to $0.6 \mathrm{Ma}$ and the other from 0.4 to the Holocene. In this Quaternary succession, the only significantly higher frequency of black tephra layers occurred at $400 \mathrm{Ka}$. Figure 9 indicates the frequency and thickness of the Quaternary tephras at Site 793. A similar pattern of tephra frequency has also been seen in the volcaniclastic materials of the Miocene Shirahama formation of the south Boso Peninsula in central Japan (Kotake, 1988). Along the northern IzuBonin Arc, rhyolitic volcanism on Niijima, Kozushima, and Shikinejima islands characteristically produced white tephra layers during the Pleistocene, whereas the pyroclastic materials of Oshima, Miyakejima, and Hachijojima islands along the volcanic front of the arc were generally basaltic. 


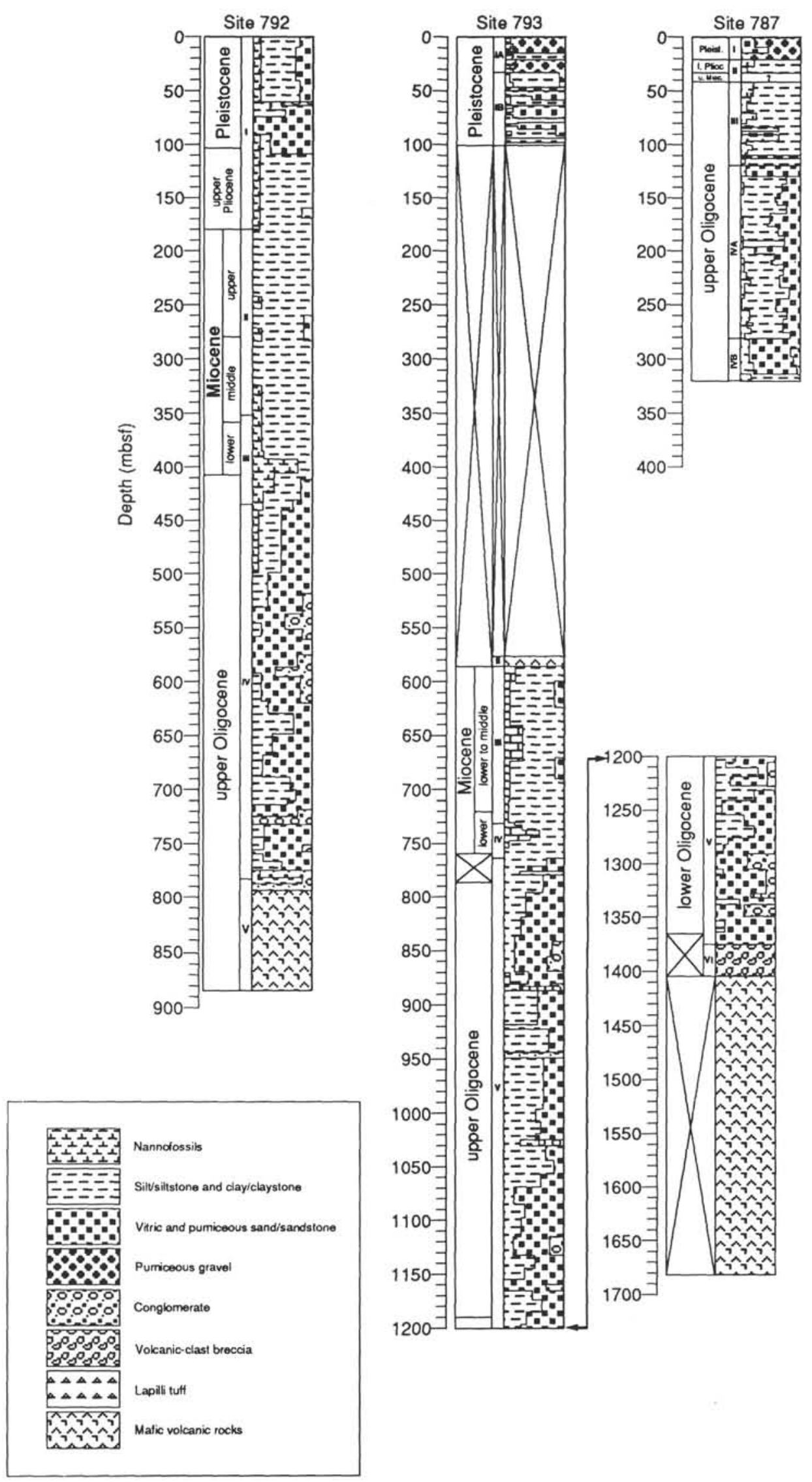

Figure 3. Lithologic columns of forearc Sites 787, 792, and 793, Izu-Bonin Arc. 

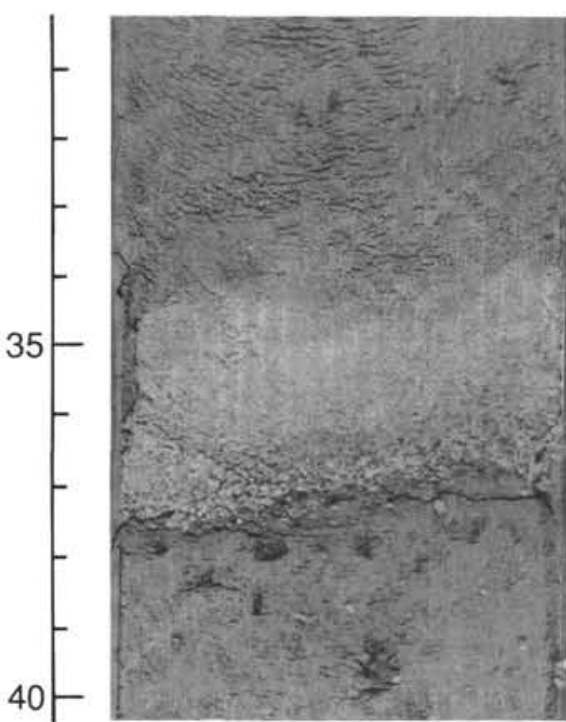

A

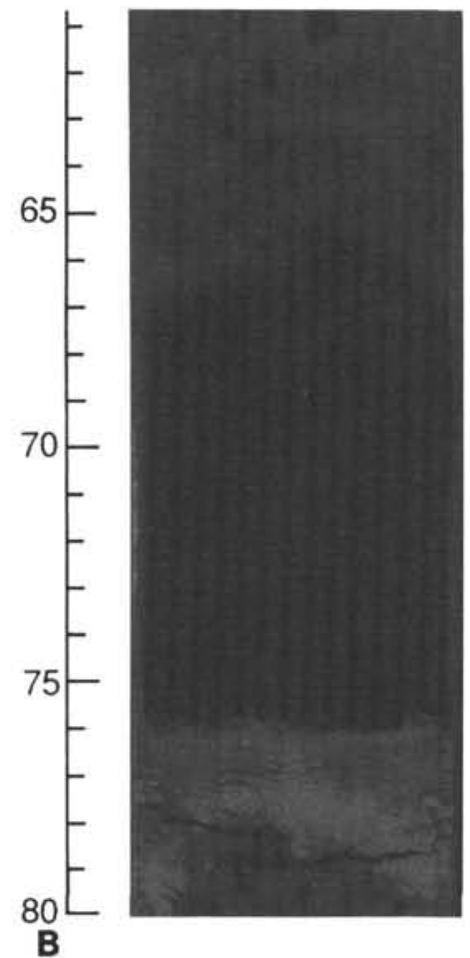

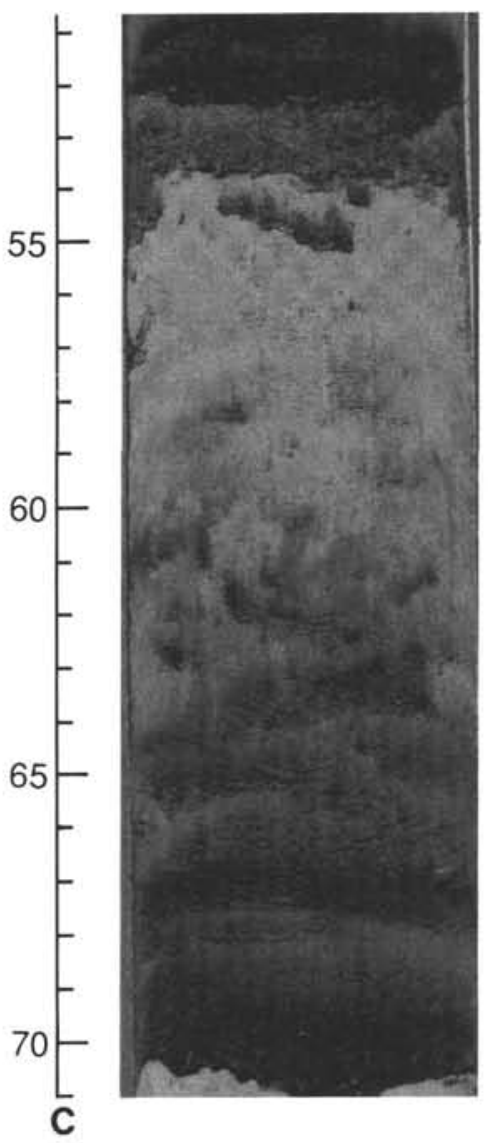

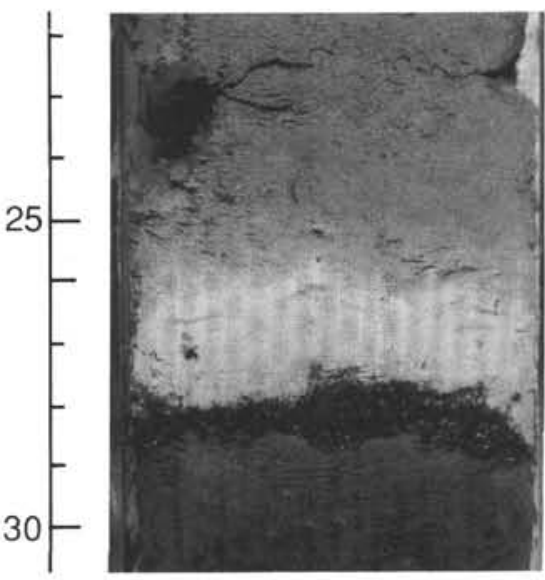

D

Figure 4. A. Simple, white tephra layer with distinct normal grading of pumice grains; Sample 126-793A-7H-3,31-38 cm. B. Multiple tephra layers consisting of medium-grained white, gray, and black tephra layers; Sample 126-792A-3H-5, 65-80 cm. C. Multiple layer consisting of two black tephra layers, the lower one distinctly laminated; Sample 126-793A-3H-2, 50-70 cm. D. Multiple layer of thin, coarse black and fine white ashes; Sample 126-792B-10X-CC, 22-30 cm.

\section{GRAIN SIZE}

Source regions and settling mechanisms of the volcanic ashes may be evaluated from grain-size data. Three types of grain-size distribution patterns are apparent (Figs. 10,11A, and 11B):

1. White, coarse tephra layers typically have median diameters of about $3 \phi$, are well sorted, and are fairly unskewed.

2. Layers of fine white ash show both well-sorted and unsorted cumulative curves with median diameters of $4 \phi$. Well-sorted parts are generally unskewed (Fig. 11A), whereas poorly sorted parts may be strongly skewed toward fine sizes (Fig. 11B). Well-sorted, fine tephra layers may represent ash falls, whereas those skewed toward the fine end may be the products of subaqueous volcanism (Walker, 1971; Sparks et al., 1981).

3. The grains in black tephra layers are well sorted, have median diameters of $3-4 \phi$, and are unskewed or only slightly skewed, although most layers are leptokurtic. Examples are the layers in Samples $126-792 \mathrm{~A}-3 \mathrm{H}-5,78-80 \mathrm{~cm}$, and $126-793 \mathrm{~A}-3 \mathrm{H}-2,50-52 \mathrm{~cm}$. This distribution pattern indicates proximal Izu-Bonin Arc sources, 
Table 1. Description of marine tephras at Hole 792A in the Izu-Bonin forearc.

\begin{tabular}{|c|c|c|c|c|c|}
\hline No & Core & Sec & $\begin{array}{l}\text { Interval } \\
\text { (cm) }\end{array}$ & $\begin{array}{l}\text { Th. } \\
(\mathrm{cm})\end{array}$ & Description and remarks \\
\hline 1 & 1 & 1 & $0-19$ & 19 & fine scoria(dispersed in silty clay) \\
\hline 2 & 1 & 1 & $34-42$ & $3 / 5$ & $\begin{array}{l}\text { light brownish gray(5YR6/1)/very light gray(N8);silt- } \\
\text { clay/sand }\end{array}$ \\
\hline 3 & 1 & 1 & $57-58$ & 1 & c.sand-granule scoria \\
\hline 4 & 1 & 1 & $63-64$ & 1 & c.sand-granule scoria \\
\hline 5 & 1 & 1 & $67-69$ & 1 & c.sand-granule scoria \\
\hline 6 & 1 & 1 & $80-82$ & 2 & black;f.sand \\
\hline 7 & 1 & 1 & $124-130$ & 6 & brownish black(5YR2/1);scoria lapilli(max.dia $=4 \mathrm{~mm}$ ) \\
\hline 8 & 1 & 2 & $30-34$ & 4 & medium light gray(N6);c.sand(pumice \& lithic) \\
\hline 9 & 1 & 2 & $51-79 \quad 9$ & $9 / 12 / 7$ & $\begin{array}{l}\text { light gray(N7)/dark gray(N3); silt-clay/f.sand/c.sand- } \\
\text { granule }\end{array}$ \\
\hline 10 & 1 & 2 & $120-124$ & 4 & dark gray(N3);f.sand \\
\hline 11 & 1 & 2 & $125-132$ & 7 & white;fine ash(dispersed) \\
\hline 12 & 1 & 2 & $139-140$ & 1 & $\begin{array}{l}\text { brownish black(5YR2/1);scoria v.c.sand(max.dia }=1 \\
\mathrm{~mm} \text { ) }\end{array}$ \\
\hline 13 & 1 & 3 & $4-5$ & 1 & scoria c.sand \\
\hline 14 & 1 & 3 & $24-25$ & 1 & olive gray $(5 Y 4 / 1) ; m . s a n d$ \\
\hline 15 & 1 & 3 & $73-80$ & $4 / 3$ & brownish gray(5YR4/1);silt/c.sand(pumice \& scoria) \\
\hline 16 & 1 & 3 & $126-145$ & 19 & light gray(N7)(purple);clay-f.sand(grading) \\
\hline 17 & 1 & 4 & $144-146$ & 2 & olive black $(5 Y 2 / 1)$;silt \\
\hline 18 & 1 & 5 & $15-16$ & 1 & medium light gray(N6);silt \\
\hline 19 & 1 & 5 & $28-32$ & $2 / 2$ & black/black;m.sand/scoria(max.dia=2 mm) \\
\hline 20 & 1 & 5 & $32-38$ & $4 / 2$ & medium light gray(N6)/white;clay/silt-clay \\
\hline 21 & 1 & 5 & $47-52$ & $3 / 2$ & black/black;f.sand/scoria(max.dia $=2 \mathrm{~mm}$ ) \\
\hline 22 & 1 & 5 & $60-62$ & 2 & dark gray(N3);scoria(max.dia=1 mm) \\
\hline 23 & 1 & 5 & $80-83$ & $2 / 1$ & medium light gray(N6)/gray;silt/c.sand \\
\hline 24 & 1 & 5 & $98-99$ & 1 & dark gray(N3);scoria c.sand(dispersed) \\
\hline 25 & 1 & 6 & $44-55$ & 11 & dark gray(N3);f.-c.sand(grading) \\
\hline 26 & 1 & 6 & $81-110$ & 29 & darkgray(N3);f-c.sand with granules(grading) \\
\hline 27 & 1 & 6 & $118-122$ & 4 & medium light gray(N6);f.sand \\
\hline 28 & 1 & 6 & $122-124$ & 2 & black;f.sand \\
\hline 29 & 1 & 6 & $131-132$ & 1 & black,f.-m.sand \\
\hline 30 & 1 & 6 & $144-147$ & $1 / 2$ & $\begin{array}{l}\text { grayish green(10GY5/2)/medium light gray(N6); silt- } \\
\text { clay }\end{array}$ \\
\hline 31 & 1 & 7 & $37-39$ & 2 & white;fine ash(dispersed) \\
\hline 32 & 2 & 1 & $+0-90+6$ & $60 / 14 / 16$ & medium dark gray(N4)/grayish black(N2);silt/f.sand \\
\hline 33 & 2 & 1 & $100-105$ & 5 & grayish black(N2); f.sand \\
\hline 34 & 2 & 1 & $140-150^{*}$ & $18 / 22$ & m.d.gray(N4)/light olive gray(5Y6/1);pumiceous v.c \\
\hline & 2 & 2 & $0-30$ & & sand $($ pum.max.dia $=2 \mathrm{~cm}) / \mathrm{m}$. sand \\
\hline 35 & 2 & 2 & $64-74$ & $8 / 2$ & $\begin{array}{l}\text { light olive gray }(5 \mathrm{Y} 6 / 1) / \text { grayish black(N2);coarse tuff } \\
\text { (pumice \& lithic, granules)/scoria c.sand }\end{array}$ \\
\hline 36 & 2 & 2 & $82-947$ & $7 / 3 / 2$ & $\begin{array}{l}\text { light gray(5YR6/1)/olive gray }(5 Y 4 / 1) / \text { brownish gray } \\
\text { (5YR4/1)(purple);clay/sand/silt }\end{array}$ \\
\hline 37 & 2 & 2 & $133-150^{*} 1$ & $12 / 13$ & light olive gray(5Y6/1);pumiceous lapilli(pum.max. \\
\hline & 2 & 3 & *0 & & $\begin{array}{l}\text { dia }=2 \mathrm{~cm} \text {,lithic max.dia }=5 \mathrm{~mm}) / \text { pumiceous sandy } \\
\text { lapilli(pum.max.dia }=1 \mathrm{~cm} \text { ) }\end{array}$ \\
\hline 38 & 2 & 3 & $40-46$ & 6 & dark gray(N3);silt-m.sand(grading) \\
\hline 39 & 2 & 3 & $59-64$ & 5 & light gray;clay \\
\hline 40 & 2 & 3 & $90-98$ & 8 & dark gray(N3);silt-f.sand(grading) \\
\hline 41 & 2 & 4 & $27-113^{*}$ & $98+$ & f.sand-pumiceous gravel(pum.max.dia $=1 \mathrm{~cm}$, two \\
\hline & 2 & cc & $0-12+$ & & kinds of pumice, white porous and gray dense) \\
\hline 42 & 3 & 1 & $27-39$ & 12 & gray;pumice lapilli tuff(pum.max.dia $=2 \mathrm{~cm}$ ) \\
\hline 43 & 3 & 1 & $50-52$ & 2 & medium gray(N5); lithic tuff,silt-v.f.sand(grading) \\
\hline 44 & 3 & 1 & $69-70$ & 1 & lithic tuff,c.sand \\
\hline 45 & 3 & 1 & $80-94$ & $10 / 4$ & $\begin{array}{l}\text { medium light gray(N6)/medium gray(N5); silt-v.f.sand/ } \\
\text { pumice c.sand, crystal (plagioclase) rich (dia }=1 \mathrm{~mm} \text { ) }\end{array}$ \\
\hline 46 & 3 & 1 & $108-113$ & 5 & medium gray(N5); tuff m.-v.f.sand \\
\hline 47 & 3 & 1 & $122-127$ & $4 / 1$ & medium light gary(N6)/medium gray(N5);silt/v.f.sand \\
\hline 48 & 3 & 2 & 3-6 & $1 / 2$ & grayish green (5G5/2) / light gray (N7); silt \\
\hline 49 & 3 & 2 & $25-27$ & 2 & grayish black(N2);silt \\
\hline 50 & 3 & 2 & $81-84$ & 3 & medium light gray(N6);silt \\
\hline 51 & 3 & 2 & $97-117$ & 20 & olive gray $(5 Y 4 / 1)$;clayey silt \\
\hline 52 & 3 & 2 & $147-148$ & 1 & white,fine tuff \\
\hline 53 & 3 & 3 & $55-57$ & 7 & m.d.gray(N4); silt -v.f.sand \\
\hline 54 & 3 & 3 & $65-73$ & 8 & gray:pumice lapilli(pum max dia $=2 \mathrm{~cm}$ ) \\
\hline 55 & 3 & 3 & $102-115$ & $12 / 1$ & white; silt / pumiceous c.sand(lithic tuff) \\
\hline 56 & 3 & 4 & $2-6$ & 4 & grayish black(N2);f.sand \\
\hline 57 & 3 & 4 & $17-22$ & 5 & dark gray(N3);silt. \\
\hline 58 & 3 & 4 & $77-78$ & 1 & black;v.f.sand(dispersed) \\
\hline 59 & 3 & 4 & $99-104$ & $4 / 1$ & $\begin{array}{l}\text { dark gray (N3); v.f.sand/scoria v.c.sand(max.dia }= \\
2 \mathrm{~mm} \text { ) }\end{array}$ \\
\hline
\end{tabular}


Table 1 (continued).

\begin{tabular}{|c|c|c|c|c|c|}
\hline 60 & 3 & 4 & $141-147$ & 6 & medium dark gray(N4);silt-m.sand(grading) \\
\hline 61 & 3 & 5 & $15-19$ & $2 / 2$ & $\begin{array}{l}\text { olive gray(5Y4/1)/dark gray(N3);v.f.sand/v.c.sand } \\
\text { (lithic tuff, lithic max.dia=1 mm) }\end{array}$ \\
\hline 62 & 3 & 5 & $54-57$ & $2 / 1$ & dark gray(N3);silt/scoria c.sand(max.dia=1 mm) \\
\hline 63 & 3 & 5 & $67-76$ & 9 & grayish black (N2);v.c.silt \\
\hline 64 & 3 & 5 & $78-79$ & 1 & medium light gray(N6);silt \\
\hline 65 & 3 & 5 & $110-114$ & 4 & white;fine tuff pocket \\
\hline 66 & 3 & 6 & 29-32 & 3 & light olive gray(5Y6/1); silt \\
\hline 67 & 3 & 6 & 47 & $<1$ & pumice tuff(max.dia $=1 \mathrm{~mm}$ ) \\
\hline 68 & 3 & 6 & $58-60$ & 2 & grayish black(N2); scoria c.sand(max.dia =2 mm) \\
\hline 69 & 3 & 6 & $82-88$ & $3 / 3$ & light gray(N7)/very light gray(N8);silt \\
\hline 70 & 3 & 6 & $100-115$ & 15 & medium dark gray(N4); silt-m.sand \\
\hline 71 & 3 & 7 & $16-17$ & 1 & olive gray $(5 Y 4 / 1)$; silt \\
\hline 72 & 3 & 7 & $42-45^{*}$ & 17 & light gray(N7);silt \\
\hline 73 & 3 & cc & $0-14$ & & \\
\hline 74 & 4 & 1 & $10-16$ & 6 & dark gray(N3);silt/v.f.sand \\
\hline 75 & 4 & 1 & $115-120$ & 5 & dark gray(N3);silt \\
\hline 76 & 4 & 2 & 8-15 & $4 / 3$ & olive gray $(5 Y 4 / 1) / g r a y i s h$ black(N2);silt / f.sand \\
\hline 77 & 4 & 2 & $19-23$ & $3 / 1$ & grayish black(N2);silt/c.sand \\
\hline 78 & 4 & 2 & $33-46$ & 13 & light olive gary (5Y6/1);silty/c.-m.sand \\
\hline 79 & 4 & 2 & $50-53$ & 3 & $\begin{array}{l}\text { grayish black(N2);v.c.sand with granule(max. } \\
\text { dia=4 mm) }\end{array}$ \\
\hline 80 & 4 & 2 & $73-80$ & 7 & dark gray(N3);silt - v.f.sand.(grading) \\
\hline 81 & 4 & 2 & 101 & $<1$ & dark gray(N3);lithic tuff(5 mm thick) \\
\hline 82 & 4 & 2 & $123-131$ & 8 & dark gray(N3);silt \\
\hline 83 & 4 & 3 & $10-15$ & 5 & black; c.sand (burrowed) \\
\hline 84 & 4 & 3 & $30-33$ & 3 & olive gray(5Y4/1);pumnice pebble(max.dia $=5 \mathrm{~mm}$ ) \\
\hline 85 & 4 & 3 & $50-52$ & 2 & dark gray(N3);silt \\
\hline 86 & 4 & 3 & 79 & $<1$ & dark gray(N3); silt \\
\hline 87 & 4 & 3 & $100-104$ & 4 & dark gray(N3);silt-f.sand(grading) \\
\hline 88 & 4 & 3 & $128-129$ & 1 & grayish black(N2); m.sand \\
\hline 89 & 4 & 4 & $6-7$ & 1 & light olive gray $(5 \mathrm{Y} 6 / 1)$; f.sand \\
\hline 90 & 4 & 4 & $31-32$ & 1 & gray;fine tuff \\
\hline 91 & 4 & 4 & 35 & $<1$ & white;fine tuff(dispersed) \\
\hline 92 & 4 & 4 & 39 & $<1$ & dark gray(N3);fine tuff(5 $\mathrm{mm}$ thick) \\
\hline 93 & 4 & 4 & $48-50$ & 2 & light olive gray $(5 Y 6 / 1) ;$;.sand \\
\hline 94 & 4 & 4 & $90-91$ & 1 & dark gray(N3);f.sand \\
\hline 95 & 4 & 4 & $94-103$ & $8 / 1$ & light olive gray(5Y6/1);silt/m.sand \\
\hline 96 & 4 & 5 & 10-17 & 7 & grayish black(N2);scoria c.sand \\
\hline 97 & 4 & 5 & $28-30$ & 2 & grayish black(N2);scoria c.sand \\
\hline 98 & 4 & 5 & $38-43$ & 5 & grayish black(N2); scoria granule \\
\hline 99 & 4 & 5 & $49-64$ & $13 / 2$ & dark gray(N3); silt / m.sand \\
\hline 100 & 4 & 5 & 81 & $<1$ & grayish black(N2);scoria c.sand(5 mm thick) \\
\hline 101 & 4 & 5 & $102-105$ & 3 & grayish black(N2);scoria c.sand(max.dia $=2 \mathrm{~mm}$ ) \\
\hline 102 & 4 & 5 & $108-110$ & 2 & grayish black(N2);scoria \\
\hline 103 & 4 & 6 & $0-2$ & 2 & grayish black(N2);scoria c.sand \\
\hline 104 & 4 & 6 & $12-15$ & 3 & light olive gray(5Y6/1); silt \\
\hline 105 & 4 & 6 & $35-36$ & 1 & grayish black(N2);f.sand -silt \\
\hline 106 & 4 & 6 & $48-50$ & 2 & dark.gray(N3); silt with scoria(max.dia=1 mm) \\
\hline 107 & 4 & 6 & $68-69$ & 1 & dark.gray(N3);m.sand \\
\hline 108 & 4 & 6 & $86-87$ & 1 & dark gray(N3);silt \\
\hline 109 & 4 & 6 & $107-108$ & 1 & dark gray(N3);silt \\
\hline 110 & 4 & 6 & $126-128$ & 2 & dark.gray(N3);silt \\
\hline 111 & 4 & 6 & $146-150$ & 4 & dark gray $(\mathrm{N} 3)$; silt \\
\hline 112 & 4 & 7 & $12-13$ & 1 & light olive gray(5Y6/1)(reddish); silt \\
\hline 113 & 4 & Cc & $12-17$ & 5 & dark.gray(N3);silt \\
\hline 114 & 5 & 1 & $+0-3$ & +3 & light olive gray $(5 Y 6 / 1)$;silt \\
\hline 115 & 5 & 1 & $18-20$ & 2 & olive gray(5Y4/1); silt \\
\hline 116 & 3 & 1 & $38-43$ & $4 /<1$ & $\begin{array}{l}\text { light olive gray(5Y6/1)/olive gray(5Y4/1); silt/ v.f.sand ( } \\
5 \mathrm{~mm} \text { thick) }\end{array}$ \\
\hline 117 & 5 & 1 & $69-72$ & 3 & $\begin{array}{l}\text { black }(5 Y 2 / 1) / \text { light olive gray }(5 Y 4 / 1) ; \text { silt/m.sand } \\
\text { pumice }(5 \mathrm{~mm} \text { thick) }\end{array}$ \\
\hline 118 & 5 & 1 & $118-119$ & 1 & very dark gray(5Y3/1);v.f.sand \\
\hline 119 & 5 & 1 & $140-142$ & 2 & olive gray(5Y4/1);silt/ v.f.sand ( $5 \mathrm{~mm}$ thick) \\
\hline 120 & 5 & 2 & $20-22$ & 2 & olive gray (5Y4/1);pumice v.c.sand \\
\hline 121 & 5 & 2 & $31-33$ & 2 & olive gray(5Y4/1);silt \\
\hline 122 & 5 & 2 & $40-44$ & 4 & light olive gray $(5 Y 6 / 1)$;silt \\
\hline 123 & 5 & 2 & $51-55$ & 4 & $\begin{array}{l}\text { very dark gray }(5 Y 3 / 1) ; m . \text { sand with scoria(max.dia } \\
=1 \mathrm{~cm})\end{array}$ \\
\hline 124 & 5 & 2 & $65-68$ & 3 & olive gray(5Y4/1); silt \\
\hline 125 & 5 & 2 & $80-89$ & $4 / 5$ & olive gray $(5 Y 4 / 1)$;silt/pumice f.sand(max.dia $=3 \mathrm{~mm}$ ) \\
\hline
\end{tabular}


Table 1 (continued).

\begin{tabular}{|c|c|c|c|c|c|}
\hline 126 & 5 & 2 & $129-130$ & 1 & olive gray(5Y4/2); silt \\
\hline 127 & 5 & 2 & $140-143$ & 3 & grayish black(N2); silt \\
\hline 128 & 5 & 3 & $8-19$ & $10 / 1$ & olive gray(5Y4/1);silt/v.f.sand \\
\hline 129 & 5 & 3 & 30 & $<1$ & black;silt(5 mm thick,dispersed) \\
\hline 130 & 5 & 3 & $54-61$ & $3 / 1 / 3$ & $\begin{array}{l}\text { light.olive gray }(5 \mathrm{Y} 6 / 1) / \text { olive gray }(5 \mathrm{Y} 4 / 1) / \text { light olive } \\
\text { gray(5Y6/1);silt/silt/fsilt with a } 5 \mathrm{~mm} \text { thick v.f.sand } \\
\text { basal part }\end{array}$ \\
\hline 131 & 5 & 3 & $75-76$ & 1 & light olive gray(5Y6/1); fine tuff \\
\hline 132 & 5 & 3 & $103-108$ & 5 & black;fine tuff (dispersed) \\
\hline 133 & $\begin{array}{l}5 \\
5\end{array}$ & $\begin{array}{l}3 \\
4\end{array}$ & $\begin{array}{l}114-150^{*} \\
0-54\end{array}$ & $11 / 79$ & light gray $(5 Y 4 / 1) /$ light(5Y $4 / 1)$;silt/clayey silt \\
\hline 134 & 6 & 1 & $14-70$ & 56 & olive gray(5Y4/1);pumiceous pebbly sand \\
\hline 135 & 6 & 1 & 96 & $<1$ & black;silt (dispersed) \\
\hline 136 & 6 & 1 & $124-125$ & 1 & grayish black(N2);silt \\
\hline 137 & 6 & 2 & $8-26$ & $7 / 6 / 5$ & $\begin{array}{l}\text { light olive gray }(5 Y 6 / 1) / \text { ight gray(N7)/olive gray } \\
(5 Y 4 / 1) \text {;silt/silt/ pumiceous v.f.sand }\end{array}$ \\
\hline 138 & 6 & 2 & $27-28$ & 1 & olive gray $(5 Y 4 / 2) ;$ v.f.sand \\
\hline 139 & 6 & 2 & $38-45$ & $5 / 2$ & $\begin{array}{l}\text { light olive gray }(5 Y 6 / 1) / \text { dark olive gray }(5 Y 3 / 2) \text {; silt/ } \\
\text { m.sand }\end{array}$ \\
\hline 140 & 6 & 2 & $92-101$ & $6 / 3$ & olive gray(5Y4/1);silt/v.f. sand (laminated) \\
\hline 141 & 6 & 3 & $4-10$ & 6 & light olive gray(5Y6/1); silt \\
\hline 142 & 6 & 3 & $34-46$ & $10 / 2$ & grayish black(N2);f.sand/v.f.sand (laminated) \\
\hline 143 & 6 & 3 & $58-68$ & 10 & $\begin{array}{l}\text { brownish black }(5 Y R 2 / 1) \text {; silt with scoria scatterd basal } \\
\text { part(max.dia }=5 \mathrm{~mm} \text { ) }\end{array}$ \\
\hline 144 & 6 & 3 & $114-136$ & $5 / 17$ & $\begin{array}{l}\text { dark olive gray(5Y3/2); grayish black(N2) silt / silt- } \\
\text { f.sand (grading) }\end{array}$ \\
\hline 145 & 6 & 4 & $3-10$ & $1 / 6$ & olive gray(5YR4/1)/olive gray(5Y4/2);silt/clayey silt \\
\hline 146 & 6 & 4 & $21-24$ & 3 & light olive gray $(5 Y 6 / 1)$;silt pocket \\
\hline 147 & 6 & 4 & $34-85$ & $4 / 47$ & $\begin{array}{l}\text { dark olive gray }(5 \mathrm{Y} 3 / 2) \text {; grayish black(N2); silt / m.- } \\
\text { f.sand }\end{array}$ \\
\hline 148 & 6 & 4 & $93-104$ & $10 / 1$ & light olive gray $(5 Y 6 / 1) /$ olive gray(5Y4/1);silt/v.f.sand \\
\hline 149 & 6 & 4 & $114-120$ & 6 & light olive gray $(5 Y 6 / 1)$;silt \\
\hline 150 & $\begin{array}{l}6 \\
6\end{array}$ & $\begin{array}{l}4 \\
5\end{array}$ & $\begin{array}{l}140-150^{*} \\
* 0-7\end{array}$ & 17 & $\begin{array}{l}\text { dark olive gray }(5 Y 3 / 2) / o l i v e \text { black(5Y2/1); clayey silt / } \\
\text { silt }\end{array}$ \\
\hline 151 & 6 & 5 & $14-13511$ & $13 / 5 / 17+$ & grayish black(N2);f.sand with pumice m-c.sand/silt/ \\
\hline & 6 & CC & *0-14+ & 14 & m.sand \\
\hline 152 & 7 & 1 & $+0-1$ & +1 & olive black(5Y2/1);v.f.sand \\
\hline 153 & 7 & 1 & $12-13$ & 1 & olive gray(5Y4/1);v.f.sand \\
\hline 154 & 7 & 1 & $21-29$ & 8 & light olive gray $(5 \mathrm{Y} 6 / 1)$; silt \\
\hline 155 & 7 & 1 & $40-573$ & $3 / 6 / 3 / 5$ & $\begin{array}{l}\text { greenish gray(5GY6/1)/olive black(5Y2/1)/light olive } \\
\text { gray }(5 Y 6 / 1) / \text { olive gray }(5 Y 4 / 1) \text {;silt/v.t.sand/silt/sandy } \\
\text { silt }\end{array}$ \\
\hline 156 & 7 & 1 & 103-131 & 28 & grayish black(N2); alternation of f.sand and v.f.sand \\
\hline 157 & 7 & 2 & $19-21$ & 2 & light olive gray $(5 Y 6 / 1)$;silt pocket \\
\hline $\begin{array}{l}158 \\
159\end{array}$ & 7 & $\frac{2}{2}$ & $41-52$ & 11 & light olive gray (5Y6/1); silt \\
\hline $\begin{array}{l}159 \\
160\end{array}$ & 7 & $\begin{array}{l}2 \\
2\end{array}$ & $\begin{array}{l}112-114 \\
119-121\end{array}$ & $\begin{array}{l}2 \\
2\end{array}$ & $\begin{array}{l}\text { grayish black (N2); v.f.sand pocket } \\
\text { grayish black(N2);v.f.sand }\end{array}$ \\
\hline 161 & 7 & 3 & $12-14$ & 2 & brownish black(5YR2/1);v.f.sand \\
\hline 162 & 7 & 3 & $27-32$ & 5 & olive gray(5YR4/1); v.f.sand \\
\hline 163 & 7 & 3 & $38-39$ & 1 & light olive gray(5Y6/1); silt \\
\hline 164 & 7 & 3 & $46-48$ & 2 & grayish black(N2); scoria sand pocket \\
\hline 165 & 7 & 3 & $57-67$ & $3 / 5 / 2$ & $\begin{array}{l}\text { dark reddish brown(5YR2/1)/dark reddish brown } \\
(5 Y R 2 / 1) ; v . f . s a n d / s a n d y \text { mud with scattered pumice } \\
\text { (max.dia }=5 \mathrm{~mm} \text { )/v.f.sand }\end{array}$ \\
\hline 166 & 7 & 3 & $81-86$ & $3 / 2$ & $\begin{array}{l}\text { dark reddish brown(5YR2/1)/grayish black(N2); silt/ } \\
\text { f.sand(laminated) }\end{array}$ \\
\hline 167 & $\begin{array}{l}7 \\
7\end{array}$ & $\begin{array}{l}3 \\
4\end{array}$ & $\begin{array}{l}98-114^{*} \\
{ }^{*} 0-56\end{array}$ & 72 & light olive gray $(5 \mathrm{Y} 6 / 1)$;silt \\
\hline 168 & 7 & 4 & $87-92$ & $1 / 1 / 3$ & $\begin{array}{l}\text { brownish black(5YR2/1);silt /f.sand(pumice } \& \text { forams } \\
\text { included)/v.f.sand with thin }(2 \mathrm{~mm} \text { ) scoria base }\end{array}$ \\
\hline 169 & 7 & 4 & $101-106$ & $3 / 2$ & $\begin{array}{l}\text { brownish black(5YR2/1)/grayish black(N2);silt/ } \\
\text { v.f.sand }\end{array}$ \\
\hline 170 & 7 & 4 & $143-145^{*} 6$ & $6 / 146 / 30+$ & dark reddish brown(5YR3/2)/grayish black(N2);clayey \\
\hline & $\begin{array}{l}7 \\
7\end{array}$ & $\begin{array}{l}5 \\
6\end{array}$ & $\begin{array}{l}* 0-150 * * \\
* 0-16 * *\end{array}$ & & ravel/may dis-10 mm)/ \\
\hline & 7 & cc & $* * 0-14+$ & & \\
\hline 171 & 8 & 1 & $+0-6$ & +6 & grayish black(N2);scoria m.sand \\
\hline 172 & 8 & 1 & $33-42$ & 9 & grayish black(N2); v.f.sand \\
\hline 173 & 8 & 1 & $48-55$ & 7 & grayish black(N2);scoria c.sand \\
\hline 174 & 8 & 1 & 75-77 & 2 & grayish black(N2);m.sand \\
\hline
\end{tabular}


Table 1 (continued).

\begin{tabular}{|c|c|c|c|c|c|}
\hline \multirow{3}{*}{$\begin{array}{l}175 \\
176\end{array}$} & 8 & 1 & $85-86$ & \multirow{3}{*}{$\begin{array}{c}1 \\
6 / 47\end{array}$} & \multirow{3}{*}{$\begin{array}{l}\text { grayish black(N2);m.sand } \\
\text { dark olive gray(5Y3/2)/olive black(5Y2/1);clayey silt / } \\
\text { v.f.sand(m.sand pumice included) }\end{array}$} \\
\hline & 8 & 1 & $131-137^{*}$ & & \\
\hline & 8 & 2 & $\cdot 0-47$ & & \\
\hline 177 & 8 & 2 & $66-83$ & $2 / 12 / 3$ & $\begin{array}{l}\text { dark reddish brown(5YR2/1)/olive black(5Y2/1)/ } \\
\text { grayish black(N2);silt/v.f.sand/sandy silt }\end{array}$ \\
\hline 178 & 8 & 2 & $93-135$ & $4 / 38$ & $\begin{array}{l}\text { brownish black(5YR2/1)/olive black(5Y2/1); silt/f.-m. } \\
\text { sand (grading)(pumice and forams included) }\end{array}$ \\
\hline 179 & 8 & 2 & $135-148$ & 13 & $\begin{array}{l}\text { scoria pebble (max.dia }=7 \mathrm{~mm} \text { ) scattered in dark olive } \\
\text { gray sandy mud) }\end{array}$ \\
\hline 180 & 8 & 2 & $148-150$ & 2 & grayish black(N2);m.sand \\
\hline 181 & 8 & 3 & $10-12$ & 2 & pumice granule \\
\hline 182 & 8 & 3 & $19-21$ & 2 & pumice granule \\
\hline 183 & 8 & 3 & 25 & $<1$ & pumice granule, seam \\
\hline 184 & 8 & 3 & 30 & $<1$ & pumice granule, seam \\
\hline 185 & 8 & 3 & $39-43^{*}$ & $9 / 11+$ & light olive gray $(5 \mathrm{Y} 6 / 1) /$ olive black(5Y2/1);sandy silt/ \\
\hline 186 & 8 & cc & $0 *-16+$ & & m.sand \\
\hline 187 & 9 & 1 & $+0-50$ & +50 & olive black(5Y2/1);scoria pebble-granule gravel \\
\hline 188 & 9 & 1 & 60 & $<1$ & m.sand (pumice) seam \\
\hline 189 & 9 & 1 & $60-78$ & 18 & olive gray $(5 Y 4 / 2)$; silt \\
\hline 190 & 9 & 2 & $29-65$ & 36 & olive black(5Y2/1);f.sand - v.f.sand (grading) \\
\hline 191 & 9 & 2 & $84-99$ & 15 & olive black(5Y2/1);v.t.sand \\
\hline 192 & 9 & 2 & $113-150^{*}$ & $3 / 37$ & olive black(5Y2/1); silt \\
\hline & 9 & 3 & $* 0-3$ & & olive black(5Y2/1);m.sand (laminated) \\
\hline 193 & 9 & 3 & $37-54$ & 17 & light olive gray(5Y6/1);silt \\
\hline 194 & $\begin{array}{l}9 \\
9\end{array}$ & $\begin{array}{l}3 \\
4\end{array}$ & $\begin{array}{ll}54-87^{*} \\
{ }^{*} 4-64\end{array}$ & 93 & olive gray $(5 Y 4 / 1)$;v.f.sand \\
\hline 195 & 9 & cc & 7 & 1 & light olive gray(5Y6/1);silt pocket \\
\hline 196 & 10 & 1 & $+0-7$ & +7 & grayish black(N2);scoria granule \\
\hline 197 & 10 & 1 & $16-18$ & 2 & grayish black(N2);scoria granule \\
\hline 198 & 10 & 1 & $36-100$ & 64 & $\begin{array}{l}\text { olive gray }(5 Y 4 / 1) \text {;pumiceous granule-c.sand (pumice } \\
\text { pebble concentrated at } 65-68,72-73 \text {, and } 85-87 \mathrm{~cm} \text { ) }\end{array}$ \\
\hline 199 & 10 & 1 & $132-148$ & 16 & olive gray(5Y5/2);pumiceous m.sand(laminated) \\
\hline 200 & 10 & 2 & $6-8$ & 2 & olive gray(5Y4/1); silt \\
\hline 201 & $\begin{array}{l}10 \\
10\end{array}$ & $\begin{array}{l}2 \\
3\end{array}$ & $\begin{array}{c}29-31^{*} \\
4^{*}-8\end{array}$ & $2 / 4$ & grayish black(N2);scoria m.sand/scoria granule \\
\hline 202 & 10 & 3 & $18-34$ & $12 / 4$ & light olive gray $(5 Y 6 / 1)$;silt/f.sand \\
\hline 203 & 10 & 3 & $39-44$ & 5 & light olive gray $(5 \mathrm{Y} 6 / 1)$; silt \\
\hline 204 & 10 & 3 & 78 & $<1$ & medium dark gray(N4);v.f.sand \\
\hline 205 & 10 & 3 & 93 & $<1$ & medium gray(N5); silty clay \\
\hline 206 & 10 & 3 & 95 & $<1$ & grayish black(N2); silt \\
\hline 207 & 10 & 3 & $118-124$ & 6 & olive black(5YR2/1);scoria granules \\
\hline 208 & 10 & 3 & $129-132$ & 3 & olive black(5YR2/1);f.sand \\
\hline 209 & 10 & 3 & $144-145$ & 1 & medium dark gray(N4); silt \\
\hline 210 & 10 & 4 & $0-5$ & 5 & grayish black(N2);v.f.sand \\
\hline 211 & 10 & 4 & $24-84$ & $16 / 44$ & $\begin{array}{l}\text { olive gray }(5 Y 4 / 2) / \text { olive black (5Y2/1); pumiceous } \\
\text { m.sand/ pumiceous m.sand (scoria concentrated) }\end{array}$ \\
\hline 212 & 10 & $\begin{array}{l}4 \\
5\end{array}$ & $\begin{array}{c}101-150^{*} \\
0-40^{* *}\end{array}$ & $109+$ & $\begin{array}{l}\text { olive black(5Y2/1);pumiceous m.sand(pum.max.dia } \\
=6 \mathrm{~mm} \text {, laminated in the upper part) }\end{array}$ \\
\hline & & $\mathrm{cc}$ & $* 0-20+$ & & \\
\hline
\end{tabular}

Notes: Data after Taylor, Fujioka, et al., 1990. Abbreviations are as follows: v.f. sand = very fine sand; $f$. sand $=$ fine sand; f. $-\mathrm{m}$. sand = fine to medium sand; $f$. $-\mathrm{c}$. sand = fine to coarse sand; $\mathrm{m}$. $-\mathrm{v} . \mathrm{f}$. sand = medium to very fine sand; $\mathrm{m}$. sand = medium sand; $\mathrm{c} . \mathrm{m}$. sand = coarse to medium sand; $\mathrm{c}$. sand = coarse sand; $\mathrm{v} . \mathrm{c}$. sand = very coarse sand; v.c. silt $=$ very coarse silt; pum. $=$ pumice; and $\max$. dia.$=$ maximum diameter. 
Table 2. Description of marine tephras at Hole 792B in the Izu-Bonin forearc.

No Core Sec $\begin{gathered}\text { Interval } \\ (\mathrm{cm})\end{gathered} \quad \begin{aligned} & \text { Th } \\ & (\mathrm{cm})\end{aligned} \quad$ Description and remarks of tephra

\begin{tabular}{|c|c|c|c|c|c|}
\hline 1 & 2 & 1 & $+0-28$ & +28 & dark gray(N3);granule-pebble \\
\hline 2 & 2 & 1 & $35-50$ & 15 & dark gray(N3); silt/f.sand \\
\hline 3 & 2 & 1 & $55-90$ & 35 & dark gray (N3)/olive gray $(5 \mathrm{Y} 4 / 1)$;silt/m.sand(grading \\
\hline 4 & 2 & 1 & $120-122$ & 2 & light gray(N7);silt \\
\hline \multirow[t]{2}{*}{5} & 2 & 1 & $140-150^{*}$ & $10 / 10$ & dark gray(N3)/grayish black(N2);m.sand/m.sand \\
\hline & 2 & 2 & $0-10$ & & \\
\hline 6 & 2 & 2 & $17-30$ & 13 & light gray(N7);silt \\
\hline 7 & 2 & 2 & $40-45$ & 5 & light gray(N7);silt \\
\hline 8 & 2 & 2 & $72-85$ & 13 & dark gray(N3);c.sand \\
\hline \multirow[t]{2}{*}{9} & 2 & 2 & $89-150^{*}$ & $81 / 82$ & grayish black(N2)/grayish black(N2);f.sand-granule \\
\hline & 2 & 3 & "0-102 & & (grading)/m.-f.sand \\
\hline 10 & 2 & 3 & $107-112$ & 5 & silt \\
\hline 11 & 2 & 3 & $116-150$ & 34 & m.-c.sand (grading) \\
\hline 12 & 2 & 4 & $82 \cdot 130$ & $8 / 40$ & dark gray(N3)/dark gray(N3);silt/f.-m.sand(grading) \\
\hline 13 & 2 & 5 & $33-40$ & 7 & olive black(5Y2/1); silt \\
\hline 14 & 2 & 5 & $60-62$ & 2 & olive black(5Y2/1); silt \\
\hline 15 & 2 & 5 & $70-71$ & 1 & dark gray(N3); silt \\
\hline 16 & 2 & 5 & $74-76$ & 2 & dark gray(N3); silt \\
\hline 17 & 2 & 5 & $86-97$ & 11 & olive black $(5 Y 2 / 1) ; f$.-m.sand \\
\hline \multirow[t]{2}{*}{18} & 2 & 6 & $+5-150^{*}$ & $+160+$ & dark gray(N3);m.-c.sand \\
\hline & 2 & $\mathrm{cc}$ & $0-15+$ & & \\
\hline 19 & 3 & 1 & $40-55$ & 15 & grayish black(N2);scoria pebble (max.dia $=1 \mathrm{~cm}$ ) \\
\hline 20 & 3 & 1 & $67-88$ & 21 & dark gray(N3);silt-v.f.sand(grading) \\
\hline 21 & 3 & 1 & $120-121$ & 1 & scoria (v.c.sand size) \\
\hline 22 & 3 & 2 & $7-47$ & $10 / 30$ & $\begin{array}{l}\text { olive black(5Y2/1)/grayish black(N2);silt/m.-v.c.sand } \\
\text { (grading) }\end{array}$ \\
\hline 23 & 3 & 2 & $54-64$ & 10 & dark gray(N3);sand with granule \\
\hline 24 & 3 & 2 & $76-92$ & $4 / 12$ & black(N1)/black(N1);silt/f.sand \\
\hline 25 & 3 & 2 & $100-125$ & $7 / 18$ & olive black(5Y2/1)/grayish black(N2); silt/f.sand \\
\hline \multirow[t]{2}{*}{26} & 3 & 2 & $137-150^{*}$ & $7 / 41$ & olive black(5Y2/1)/grayish black(N2);silt/f.sand \\
\hline & $\begin{array}{l}3 \\
3\end{array}$ & $\begin{array}{l}3 \\
3\end{array}$ & $0-41$ & & \\
\hline 27 & 3 & 3 & $70-95$ & 7/8/10 & $\begin{array}{l}\text { grayish black(N2)/very light gray(N8)/olive black } \\
2 / 1 \text { );.sand(grading)/silt/silt-v.f.sand(grading) }\end{array}$ \\
\hline 28 & 3 & 3 & $117-118$ & 1 & v.c.sand \\
\hline \multirow[t]{2}{*}{29} & 3 & 3 & $145-150^{*}$ & $5 / 5$ & olive black(5Y2/1)/grayish black(N2); silt/sand \\
\hline & 3 & 4 & $0-5$ & & \\
\hline 30 & 3 & 4 & $26-29$ & 3 & scoria granule(max.dia $=4 \mathrm{~mm}$ ) \\
\hline 31 & 3 & 4 & $59-61$ & 2 & grayish green(10GY5/2); silt \\
\hline 32 & 3 & 4 & $70-1155$ & $5 / 10 / 30$ & $\begin{array}{l}\text { olive black(5Y2/1)/olive black(5Y2/1)/grayish black } \\
\text { (N2);silt/v.f.sand/v.f.sand }\end{array}$ \\
\hline 33 & 3 & 4 & $147-150^{*}$ & $3 / 30$ & olive black(5Y2/1)/grayish black(N2);silt/sand \\
\hline & 3 & 5 & ${ }^{*} 0-30$ & & \\
\hline 34 & 3 & 5 & $50-95$ & $10 / 35$ & $\begin{array}{l}\text { olive black }(5 Y 2 / 1) / \text { grayish black(N2);silt/sand with } \\
\text { granule at base }\end{array}$ \\
\hline 35 & 3 & 5 & $111-112$ & 1 & scoria v.c.sand \\
\hline 36 & 3 & 5 & $127-150$ & 23 & olive gray $(5 Y 4 / 1)$;f.sand \\
\hline 37 & 3 & 6 & $15-501$ & 15/8/12 & $\begin{array}{l}\text { brownish gray(5YR4/1)/olive gray }(5 Y 4 / 1) / \text { brownish } \\
\text { gray(5YR } 4 / 1) \text { (pinkish);f.sand }\end{array}$ \\
\hline 38 & 4 & 1 & $11-20$ & 9 & light gray(N7);silt \\
\hline 39 & 4 & 1 & $46-49$ & 3 & medium dark gray(N4);f.sand \\
\hline 40 & 4 & 1 & $54-55$ & 1 & medium gray(N5);silt \\
\hline 41 & 4 & 1 & $55-58$ & 3 & very dark gray (N3),f.sand \\
\hline 42 & 4 & 1 & $60-62$ & 2 & medium gray(N5);silt \\
\hline 43 & 4 & 1 & $88-90$ & 2 & dark gray(N3);silt \\
\hline 44 & 4 & 1 & $113-115$ & 2 & olive black(5Y2/1); \\
\hline 45 & 4 & 1 & $119-120$ & 1 & olive black $(5 Y 2 / 1) ; c$.sand \\
\hline 46 & 4 & 2 & $26-42$ & $6 / 6 / 4$ & $\begin{array}{l}\text { dark gray(N3)/olive black(5Y2/1)/dark gray(N3);silt/ } \\
\text { clayey silt/silt }\end{array}$ \\
\hline 47 & 4 & 2 & $63-66$ & 3 & dark gray(N3);silt \\
\hline 48 & 4 & 2 & 73 & $<1$ & dark gray(N3);c.sand(5 mm thick) \\
\hline 49 & 4 & 2 & $84-90$ & $1 / 2 / 3$ & $\begin{array}{l}\text { dark gray }(\mathrm{N} 3) /(\mathrm{N} 3) /(\mathrm{N} 3) \text {;clay/sand scoria c.sand } \\
\text { (max.dia }=4 \mathrm{~mm} \text { ) }\end{array}$ \\
\hline 50 & 4 & 2 & $101-109$ & $2 / 5 / 1$ & $\begin{array}{l}\text { dark gray(N3)/dark gray(N3)/pale olive(10Y6/2); } \\
\text { c.sand/scoria f.sand/scoria (max.dia }=4 \mathrm{~mm} \text { ) }\end{array}$ \\
\hline & 4 & 2 & $122-137$ & $10 / 5$ & dark gray(N3)/dark gray(N3);silt/c.sand(grading) \\
\hline 52 & 4 & 3 & $1-3$ & 2 & dark gray(N3);silt \\
\hline 53 & 4 & 3 & $22-30$ & $4 / 4$ & greenish gray $(5 \mathrm{Y} 6 / 1) /$ light gray(N7); silt/silt \\
\hline 54 & 4 & 3 & $60-61$ & 1 & dark gray(N3);m.sand \\
\hline 55 & 4 & 3 & $85-87$ & 2 & olive gray $(5 Y 4 / 1)$; silt \\
\hline 56 & 4 & $c c$ & $28-30+$ & $2+$ & light gray(N7);silt \\
\hline 57 & 5 & CC & $+0-15$ & +15 & $\begin{array}{l}\text { greenish gray }(5 \mathrm{GY} 6 / 1) \text {; scoria granule in mud ( } \max \text {. } \\
\text { dia }=5 \mathrm{~mm} \text { ) }\end{array}$ \\
\hline
\end{tabular}


Table 2 (continued).

\begin{tabular}{|c|c|c|c|c|c|}
\hline 58 & 5 & $\mathrm{cc}$ & $15-29$ & 14 & olive gray(5Y4/1);f.sand \\
\hline & & $\mathrm{cc}$ & $+0-13+$ & $+13+$ & $\begin{array}{l}\text { dark gray }(\mathrm{N} 3) ; \mathrm{scoria} \text { granule(scoria max.dia }=5 \mathrm{~mm} \text {, } \\
\text { pum. max.dia }=4 \mathrm{~mm} \text { ) }\end{array}$ \\
\hline 60 & 7 & $\mathrm{cc}$ & $8-12$ & 4 & dark greenish gray(5GY4/1);m.sand \\
\hline 61 & 8 & 1 & $6-7$ & 1 & olive black(5Y2/1); silty clay \\
\hline 62 & 8 & 1 & $19-26$ & 7 & olive black(5Y2/1); silty clay \\
\hline 63 & 8 & 1 & $61-63$ & 2 & olive black(5Y2/1);silty clay \\
\hline 64 & 8 & 2 & $31-32$ & 1 & very light gray(N7);fine ash \\
\hline 65 & 8 & 2 & $54-55$ & 1 & light olive gray $(5 Y 6 / 1)$;silt \\
\hline 66 & 8 & 2 & $72-78$ & 6 & grayish black(N2);clay \\
\hline 67 & 8 & 2 & $86-91$ & 5 & grayish black(N2);clay \\
\hline 68 & 8 & 2 & $115-123$ & 8 & grayish black(N2);clay with Chondrites type burrow \\
\hline 69 & 8 & 3 & $18-26$ & 8 & dark gray(N3);silt(grading) \\
\hline 70 & 8 & 3 & $36-41$ & 5 & grayish black(N2);m.-c.sand(grading, max.dia $=5 \mathrm{~mm}$ ) \\
\hline 71 & 8 & 3 & $60-66$ & 6 & grayish black(N2);m.sand \\
\hline 72 & 8 & 3 & $82-85$ & 3 & olive gray $(5 Y 4 / 1)$; silt \\
\hline 73 & 8 & 4 & $40-46$ & 6 & light gray $(5 Y 6 / 1)$;silt \\
\hline 74 & 8 & 4 & $80-86$ & 6 & medium dark gray(N4); silty clay \\
\hline 75 & 8 & 4 & $110-115$ & 5 & light gray(N7); silt with m.sand base \\
\hline 76 & 8 & 4 & $123-129$ & 6 & light gray(N7);silt \\
\hline 77 & 9 & 1 & $32-33$ & 1 & light gray(N7); silt \\
\hline 78 & 9 & 1 & $99-100$ & 1 & light gray(N7);silt \\
\hline 79 & 9 & 1 & $124-130$ & 6 & light gray(N7); silt \\
\hline 80 & 9 & 2 & $27-30$ & 3 & light gray(N7);sandy silt \\
\hline 81 & 9 & 2 & $36-37^{*}$ & 2 & light gray(N7);silt \\
\hline & 9 & $c c$ & $0-1$ & & \\
\hline 82 & 9 & $\mathrm{cc}$ & $9-10$ & 1 & light gray(N7);v.f.sand \\
\hline 83 & 10 & 1 & $32-36$ & $2 / 2$ & $\begin{array}{l}\text { very dark gray }(5 Y 3 / 1) / o l i v e \text { gray(5Y4/1);silt/pumice } \\
\text { m.sand(laminated) }\end{array}$ \\
\hline 84 & 10 & 1 & $72-79$ & $3 / 4$ & light olive gray $(5 Y 6 / 2) /$ light gray $(5 Y 7 / 1)$; silt/silt \\
\hline 85 & 10 & 1 & $85-93$ & $4 / 3 / 1$ & $\begin{array}{l}\text { olive gray }(5 Y 4 / 2) / \text { olive gray }(5 Y 4 / 2) / v e r y \text { dark gray } \\
(5 Y 3 / 1) ; p u m i c e ~ g r a n u l e(m a x . d i a=3 \mathrm{~mm}) / \mathrm{silt} / \mathrm{v} \text {.f.sand } \\
\text { (laminated) }\end{array}$ \\
\hline 86 & 10 & 1 & $107-109$ & 2 & very dark gray $(5 Y 3 / 1)$;c.sand \\
\hline 87 & 10 & 1 & $135-136$ & 1 & very dark gray $(5 Y 3 / 1)$; silt \\
\hline 88 & 10 & 2 & $20-21$ & 1 & olive gray(5Y5/2); silt \\
\hline 89 & 10 & 2 & $46-51$ & 5 & lolive gray $(5 \mathrm{Y} 4 / 1)$; pumice m.sand(max.dia $=4 \mathrm{~mm}$ ) \\
\hline 90 & 10 & 2 & $81-84$ & 3 & olive gray (5Y5/2);silt \\
\hline 91 & 10 & 2 & $101-103$ & $1 / 1$ & $\begin{array}{l}\text { olive gray }(5 Y 5 / 2) / v e r y \text { dark gray(5Y3/1);silt/pumice } \\
\text { m.sand }\end{array}$ \\
\hline 92 & $\begin{array}{l}10 \\
10\end{array}$ & $\begin{array}{l}2 \\
3\end{array}$ & $\begin{array}{c}147-150^{*} \\
0-6\end{array}$ & $7 / 2$ & olive gray $(5 Y 4 / 2) / g r a y(5 Y 5 / 1)$;silt/silt \\
\hline 93 & 10 & 3 & $15-19$ & 4 & gray $(5 Y 5 / 1)$; silt with f.sand at base $(5 \mathrm{~mm}$ thick) \\
\hline 94 & 10 & 3 & 33-36 & $1 / 2$ & $\begin{array}{l}\text { gray }(5 Y 5 / 1) / 0 l i v e \text { gray }(5 Y 4 / 2) \text {; silt/pumice v.c.sand } \\
\text { (max.dia }=5 \mathrm{~mm} \text { ) }\end{array}$ \\
\hline 95 & 10 & 3 & $47-51$ & $2 / 1 / 2$ & $\begin{array}{l}\text { gray }(5 Y 5 / 1) / v e r y \text { dark gray }(5 Y 3 / 1) / g r a y(5 Y 5 / 1) \text {; silt/ } \\
\text { silt/silt }\end{array}$ \\
\hline 96 & 10 & 3 & $61-62$ & 1 & gray(5Y5/1); silt \\
\hline 97 & 10 & 3 & $109-114$ & 5 & light olive gray $(5 Y 6 / 1)$;silt \\
\hline 98 & 10 & cc & $26-33$ & $2 / 1 / 4$ & $\begin{array}{l}\text { light olive gray }(5 Y 6 / 1) / g r a y i s h \text { black(N2)/brownish } \\
\text { black(5YR2/1);silt/f.sand/silty clay }\end{array}$ \\
\hline 99 & 11 & 1 & $13-15$ & 2 & gray(5Y5/1); silt \\
\hline 100 & 11 & 1 & $47-49$ & $1 / 1$ & $\begin{array}{l}\text { gray }(5 Y 5 / 1) /(5 Y 5 / 1) ; f \text { sand/silt with scoria } c . \text { sand at } \\
\text { base }(5 \mathrm{~mm} \text { thick, max.dia }=2 \mathrm{~mm})\end{array}$ \\
\hline 101 & 11 & 1 & $65-66$ & 1 & light olive gray $(5 Y 6 / 1)$;silt \\
\hline 102 & 11 & 2 & $0-13$ & $1 / 4 / 2 / 6$ & $\begin{array}{l}\text { light olive gray/olive gray }(5 \mathrm{Y} 4 / 2) /(5 \mathrm{Y} 4 / 2) / \text { grayish } \\
\text { black(N2);silt/silt with scoria/m.sand scattered in silty } \\
\text { clay/silt with scoria(max.dia }=3 \mathrm{~mm} \text { ) }\end{array}$ \\
\hline 103 & 11 & 2 & 42 & $<1$ & black;f.sand seam (5 mm thick) \\
\hline 104 & 11 & 2 & $48-5$ & $3 / 6$ & $\begin{array}{l}\text { gray(5Y5/1)/light olive gray }(5 Y 6 / 1) \text {; silt with pumice } \\
\mathrm{m} . \text { sand/silt }\end{array}$ \\
\hline 105 & 11 & 2 & $83-88$ & 5 & olive gray(5Y5/1);silt \\
\hline 10 & 11 & $c c$ & & $2 / 3 / 1$ & $\begin{array}{l}\text { grayish black(N2)/olive gray(5Y4/1)/dark olive gray } \\
(5 Y 3 / 2) \text {;silt/f.sand/f.sand }\end{array}$ \\
\hline 107 & 11 & cc & $27-31$ & 4 & $\begin{array}{l}\text { grayish black(N2);silt } \\
\text { SITE } 792 \mathrm{E}\end{array}$ \\
\hline
\end{tabular}

$\begin{array}{ccccc}1 & 2 & 1 & 17 & <1 \\ 2 & 3 & 1 & +0-7 & +3 / 4 \\ 3 & 3 & 1 & 15 & <1 \\ 4 & 3 & 1 & 21-23 & 2 \\ 5 & 3 & 1 & 41-46 & 1 / 2 / 2\end{array}$

reddish gray(10R5/1); silt, pocket olive gray $(5 Y 5 / 2) / l i g h t$ gray(N7); silt/silt with pumice at base(max.dia $=5 \mathrm{~mm}$ ) pumice $\mathrm{m}$. sand seam $(5 \mathrm{~mm}$ thick) brownish black(5YR2/1);v.f.sand olive gray(5Y4/2)/grayish black(N2)/light olive gray (5Y6/1);scoria pebble to granule/ditto with silt ( $\mathrm{max} . \mathrm{dia}=8 \mathrm{~mm}$ ) 
Table 2 (continued).

\begin{tabular}{|c|c|c|c|c|c|}
\hline 6 & 3 & 1 & 55 & 1 & black;f.sand(diiseminated) \\
\hline 7 & 3 & 1 & 59 & 1 & black,f.sand(disseminated) \\
\hline 8 & 4 & 1 & $21-22$ & 1 & olive black(5Y2/1);silt with scoria sand at base \\
\hline 9 & 4 & 1 & $80-150$ & 70 & scoria \& pumice granule scattered in clay \\
\hline 10 & 4 & 2 & $10-75$ & $15 / 50$ & $\begin{array}{l}\text { olive black( } 5 \mathrm{Y} 2 / 1) / \text { grayish black(N2); silty clay/scoria } \\
\text { silty v.c.sand(max.dia= } 4 \mathrm{~mm} \text { ) }\end{array}$ \\
\hline 11 & 5 & 1 & $25-32$ & 7 & olive gray $(5 Y 4 / 1)$;silt \\
\hline \multirow[t]{2}{*}{12} & 5 & 1 & $70-75^{*}$ & $5 / 4 / 2$ & olive $(5 Y 5 / 3) /$ olive gray $(5 Y 4 / 2) / g r a y(5 Y 5 / 1)$; silt/si.t/silt \\
\hline & 5 & cc & $0-6$ & & \\
\hline 13 & 8 & 1 & $25-34$ & $4 / 5$ & $\begin{array}{l}\text { greenish black( } 5 G Y 2 / 1) / \text { olive gray }(5 Y 4 / 1) \text {; silty clay/ } \\
\text { clayey silt }\end{array}$ \\
\hline 14 & 9 & 1 & $27-29$ & 2 & olive black(5Y2/1);silt \\
\hline 15 & 9 & 1 & $76-83$ & $2 / 5$ & olive gray $(5 Y 4 / 1) /$ olive gray $(5 Y 4 / 1)$; silt/v.t.sand \\
\hline 16 & 9 & 1 & $94-95$ & 1 & olive gray $(5 Y 4 / 1)$; silt \\
\hline 17 & 9 & 1 & $115-116$ & 1 & grayish black(N2);silt \\
\hline 18 & 9 & 1 & $144-146$ & 2 & olive black(5Y2/1);silt \\
\hline 19 & 9 & 2 & $94-96$ & 2 & dark olive gray $(5 Y 3 / 2)$; silt(laminated) \\
\hline 20 & 9 & 3 & $0-7$ & & greenish black(5GY2/1);clayey silt(laminated) \\
\hline 21 & 9 & 3 & $16-20$ & $3 / 1$ & $\begin{array}{l}\text { greenish black(5GY2/1)/grayish black(N2); clayey silt/ } \\
\text { silt(laminated) }\end{array}$ \\
\hline 22 & 9 & 3 & $96-103$ & 7 & $\begin{array}{l}\text { dark greenish gray(5G4/1);pumice granule-pebble } \\
\text { (max.dia }=10 \mathrm{~mm} \text { ) }\end{array}$ \\
\hline 23 & 9 & 3 & $110-117$ & 7 & greenish black(5GY2/1);clayey silt(laminated) \\
\hline 24 & 10 & 1 & $144-146$ & 2 & grayish black(N2);silt \\
\hline 25 & 12 & 2 & $83-90$ & 7 & $\begin{array}{l}\text { olive gray }(5 \mathrm{Y} 4 / 1) \text {;scoria pebble(max.dia }=7 \mathrm{~mm}) \\
\text { scatterred in muddy sandstone }\end{array}$ \\
\hline 26 & 16 & 2 & $6-11$ & 5 & olive black(5Y2/1);clayey silt(laminated) \\
\hline 27 & 16 & 2 & $92-98$ & 6 & olive black(5Y2/1);clayey silt(laminated) \\
\hline 28 & 17 & 1 & $50-51$ & 1 & grayish black(N2); ${ }^{\cdots *}$ \\
\hline 29 & 17 & 1 & $72-73$ & 1 & grayish black(N2); ${ }^{* * *}$ \\
\hline 30 & 17 & 1 & $85-95$ & 10 & grayish black(N2);silt-v.c.sand(grading) \\
\hline 31 & 17 & 1 & $100-107$ & 7 & grayish black(N2);silt-v.c.sand(grading) \\
\hline \multirow[t]{2}{*}{32} & 17 & 1 & $148-150^{\circ}$ & 12 & grayish black(N2)/dark olive gray(5Y3/2); silt \\
\hline & & 2 & $0-10$ & & (laminatin in lower part) \\
\hline 33 & 17 & 2 & $14-15$ & 1 & pale red(5R6/2); ${ }^{\ldots *}$ \\
\hline 34 & 17 & 2 & $16-22$ & 6 & dark olive gray $(5 \mathrm{Y} 3 / 2)$;silt(grading) \\
\hline 35 & 17 & 2 & $38-40$ & 2 & dark olive gray $(5 Y 3 / 2)$;scoria granule-fine pebble \\
\hline 36 & 17 & 2 & $51-58$ & 7 & greenish black(5GY2/1);silt/m.sand(grading) \\
\hline 37 & 17 & 2 & $66-71$ & 5 & dark olive gray $(5 Y 3 / 2)$; silt \\
\hline 38 & 17 & 2 & $119-123$ & 4 & dark olive gray $(5 Y 3 / 2)$;silty v.f.sand \\
\hline 39 & 17 & 3 & $53-55$ & 2 & light gray(N7);silt \\
\hline 40 & 17 & 4 & $19-41$ & 22 & ** clayey silt \\
\hline 41 & 18 & 1 & $48-50$ & 2 & olive black(5Y2/1);silt-f.sand(grading) \\
\hline 42 & 18 & 1 & $79-82$ & 3 & olive black(5Y2/1);silt \\
\hline 43 & 18 & 1 & $107-111$ & 4 & olive black(5Y2/1); silt \\
\hline 44 & 18 & 1 & $114-117$ & 3 & olive black(5Y2/1);f.sand \\
\hline 45 & 18 & 2 & $103-110$ & 7 & grayish black(N2);silt-f.sand(grading) \\
\hline 46 & 18 & 2 & $142-147$ & 5 & brownish gray(5YR4/1);silt-silty sand(grading) \\
\hline 47 & 18 & 3 & $20-23$ & 3 & dark greenish gray(5GY4/1);v.f.sand \\
\hline 48 & 18 & 3 & $37-44$ & 7 & olive black(5Y2/1);silt-v.f.sand(grading) \\
\hline 49 & 18 & 3 & $49-52$ & 3 & greenish gray $(5 \mathrm{GY} 6 / 1)$;f.sand \\
\hline 50 & 18 & 3 & $62-63$ & 1 & grayish black(N2);v.f.sand(laminated) \\
\hline 51 & 18 & 3 & $85-87$ & 2 & scoria granule \\
\hline 52 & 18 & 3 & $112-113$ & 1 & olive gray $(5 Y 4 / 1)$;c.sand \\
\hline 53 & 18 & 4 & $0-9$ & 9 & olive black(5Y2/1);silt \\
\hline 64 & 18 & 4 & $16-20$ & 4 & olive black(5Y2/1);silt \\
\hline 55 & 18 & 4 & $43-47$ & 4 & olive black(5Y2/1);silt-v.f.sand(grading) \\
\hline 56 & 18 & 4 & $112-114$ & 2 & olive black(5Y2/1);silt \\
\hline 57 & 18 & 4 & $116-117$ & 1 & olive black(5Y2/1);c.sand \\
\hline 58 & 18 & 4 & $120-121$ & 1 & olive black $(5 Y 2 / 1)$;v.f.sand \\
\hline
\end{tabular}

Notes: Data after Taylor, Fujioka, et al., 1990. Abbreviations are as in Table 1. 


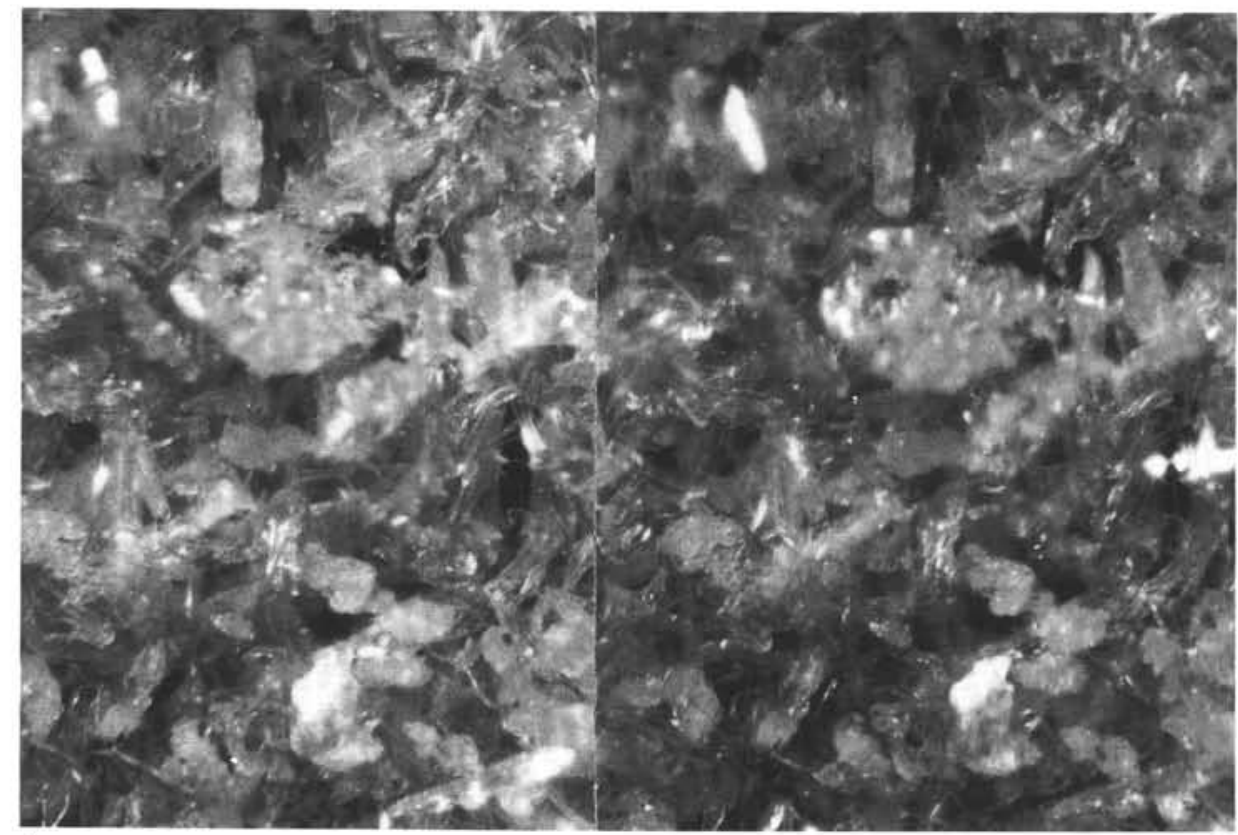

A

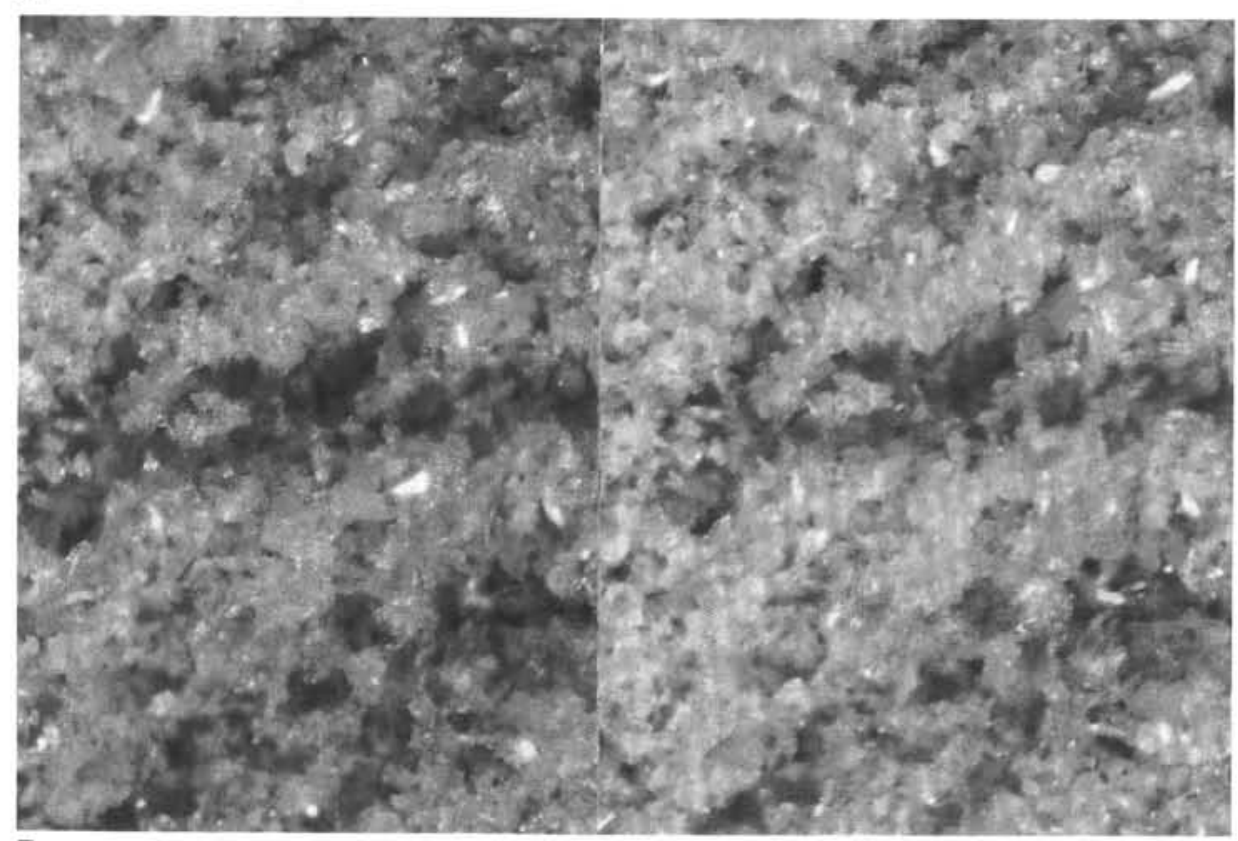

B

Figure 5. Stereographic photomicrographs of tephra layers. A. Coarser tephra with both translucent bubble-wall and pumice-type glass shards; Sample 126-792A-1H-5, 81-83 cm. B. Example of tephra composed of very fine, white, bubble-wall glass shards; Sample 126-792A-1H-1, 38-40 cm.

but more stenokurtic black tephra layers may have resulted from secondary sources, remobilized as turbidity currents and grain flows.

\section{CHEMICAL COMPOSITIONS OF THE TEPHRA LAYERS}

We analyzed a total of 1240 shards from 145 layers (2-35 analyses per layer) by electron probe (Microfiche). Our results document the chemical history of volcanism in the Izu-Bonin arc-trench system since the Oligocene. Variations in major oxides with stratigraphic position are shown in Figure 12. Separate curves are drawn for black glass shards, which normally contain $<65 \mathrm{wt} \%$ silica, and white ones, which are rhyolitic and have silica contents $>65 \mathrm{wt} \%$. Notably, the chemical compositions of the two glass types do not overlap, strongly suggesting that the black and white ashes were separate basalt and rhyolite eruptions. Most of the glasses from the black tephra layers fall into the basalt and/or basaltic andesite clans, but some are andesite. $\mathrm{TiO}_{2}$ contents are invariably $<1.5 \%$, and some are $<1 \%$. The $\mathrm{K}_{2} \mathrm{O}$ contents of most of the glass is $<0.4 \%$, values lower than those of volcanic rocks typical of island arcs (Kurosawa, 1959; Miyashiro, 1974; Kurosawa and Michino, 1976; Yuasa, 1985). The $\mathrm{K}_{2} \mathrm{O}$ contents of the colorless volcanic glass shards are especially notable. Most 


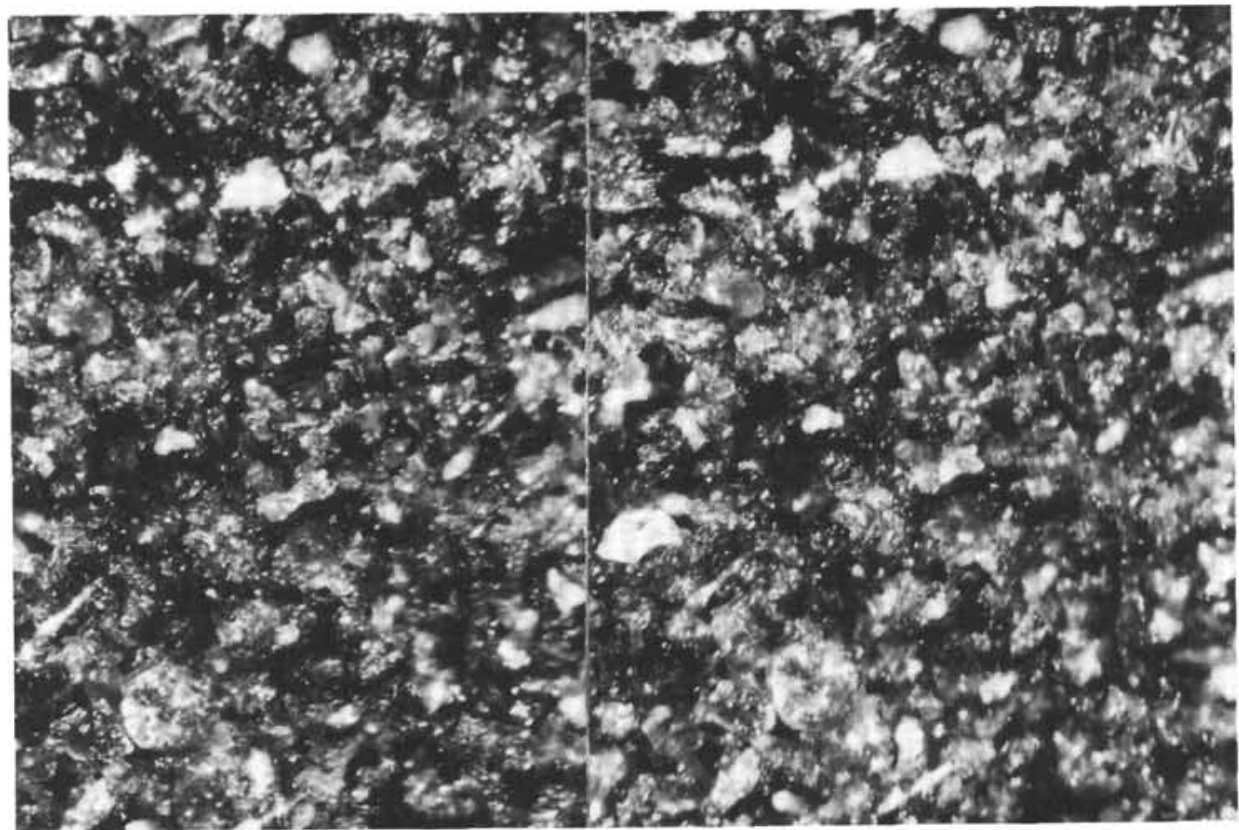

A
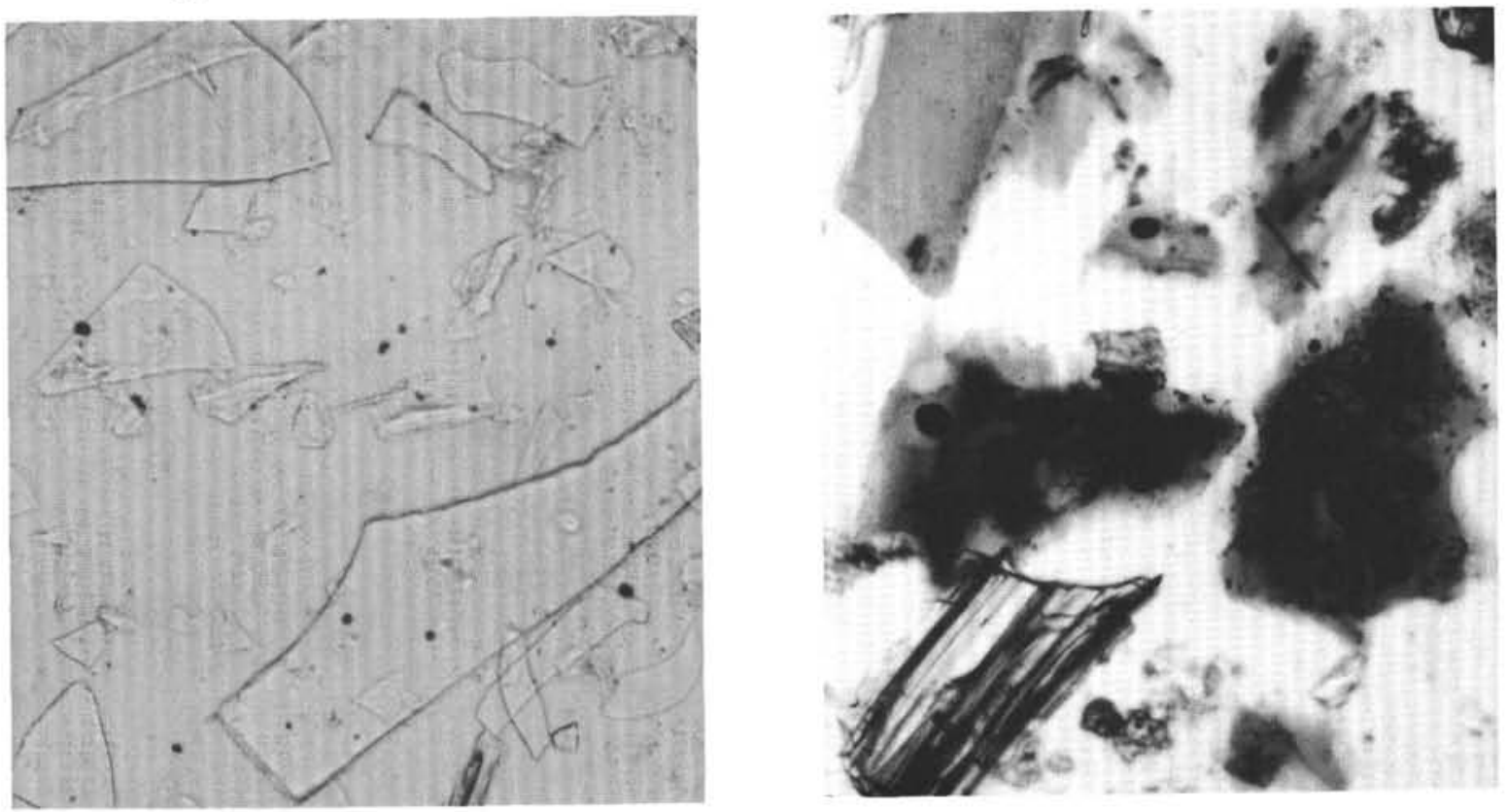

B

C

Figure 6. Photomicrographs of tephra layers. A. Stereographic photomicrograph of fine, black volcanic tephra layer having considerable amounts of lithic and crystals; Sample 126-793A-8H-5, 28-30 cm. B, C. Photomicrographs of the smear slides of the tephra layers; (B) black tephra with brown glass and clear tabular glass shards, Sample 126-792A-6H-3,130-132 cm; (C) bubble-wall-type translucent glasses, Sample 126-792A-1H-5, 81-83 cm.

values are low, generally $<1 \%$; however, several beds have shards with high $\mathrm{K}_{2} \mathrm{O}$ in the section younger than $3 \mathrm{Ma}$.

Silica values of the Miocene and Pliocene ashes are clearly bimodal (Fig. 13), as is the case in the middle Miocene northeast Japan Arc (Konda, 1974) and in the Cascade region of the northwestern United States (Christiansen and Lipman, 1972). $\mathrm{SiO}_{2}$ in Quaternary tephra layers display similar spreads; however, their basaltic, and especially their rhyolitic peaks, are dominant compared with the andesitic ones, as in the Cascade region of North America (Suga and Fujioka, 1990; Eaton, 1982, 1984).

\section{$\mathrm{SiO}_{2}$-Alkali Relationship}

Figures $14 \mathrm{~A}$ and $14 \mathrm{~B}$ present silica-alkali plots of all the data. The $\mathrm{SiO}_{2}$ contents of the shards range from 48 to $79 \mathrm{wt} \%$, but are bimodally clustered into two sets, with values centered on 54 and 72 wt\%, respectively, reflecting the definite bimodal color pattern observed visually in the cores on board the JOIDES Resolution. As noted earlier, the $65 \%$ silica value separates the white and black ashes. Most of the tephra layers belong to the low-alkali tholeiitic series of Kuno $(1960,1966)$, and some belong to the high-alkali and alkali-olivine- 


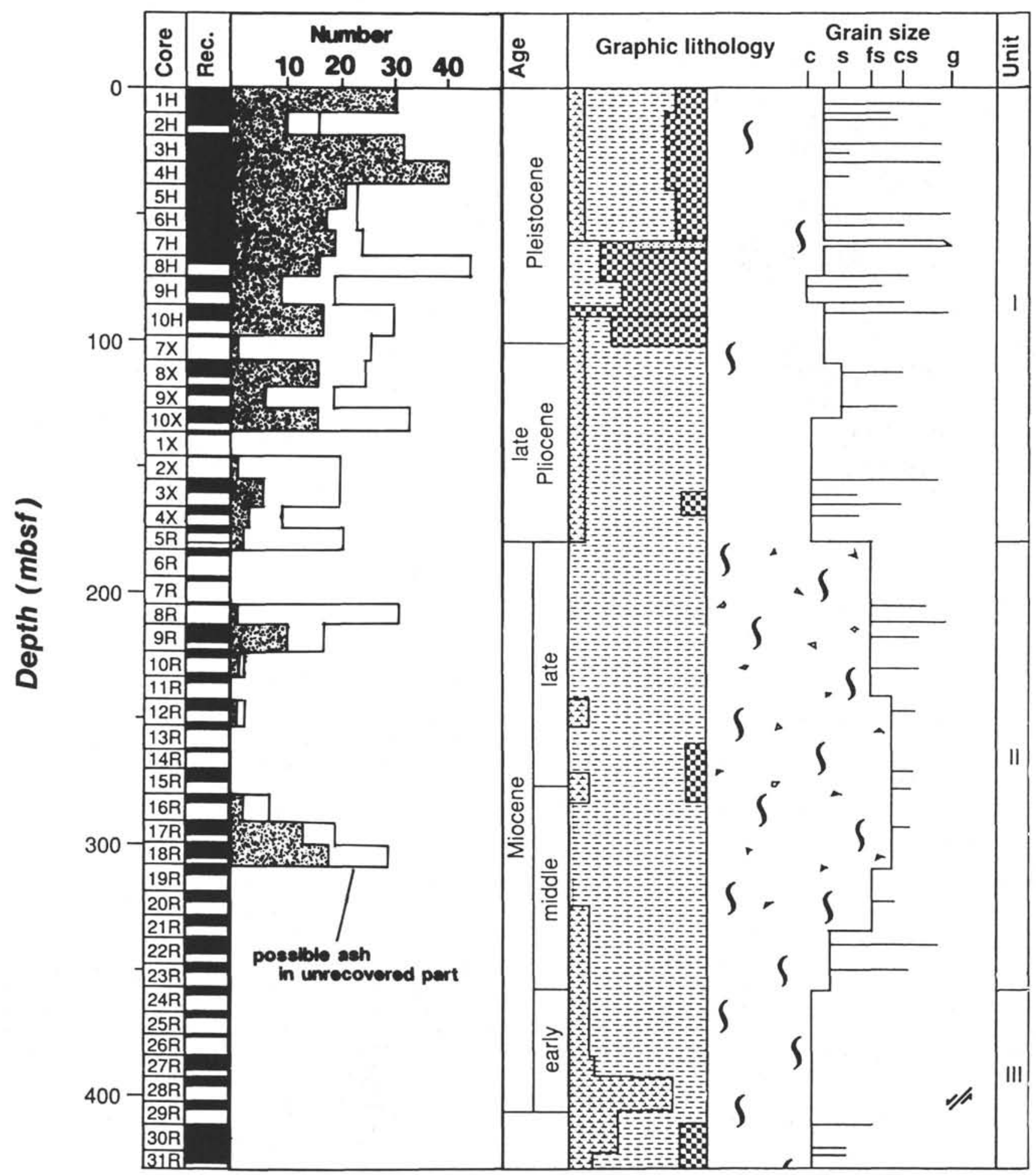

Figure 7. Frequency diagram of the tephra layers for forearc Site 792. Columns from left to right show core number, recovery rate, ash frequency, age, graphic lithology, grain size, and lithologic unit, respectively. 


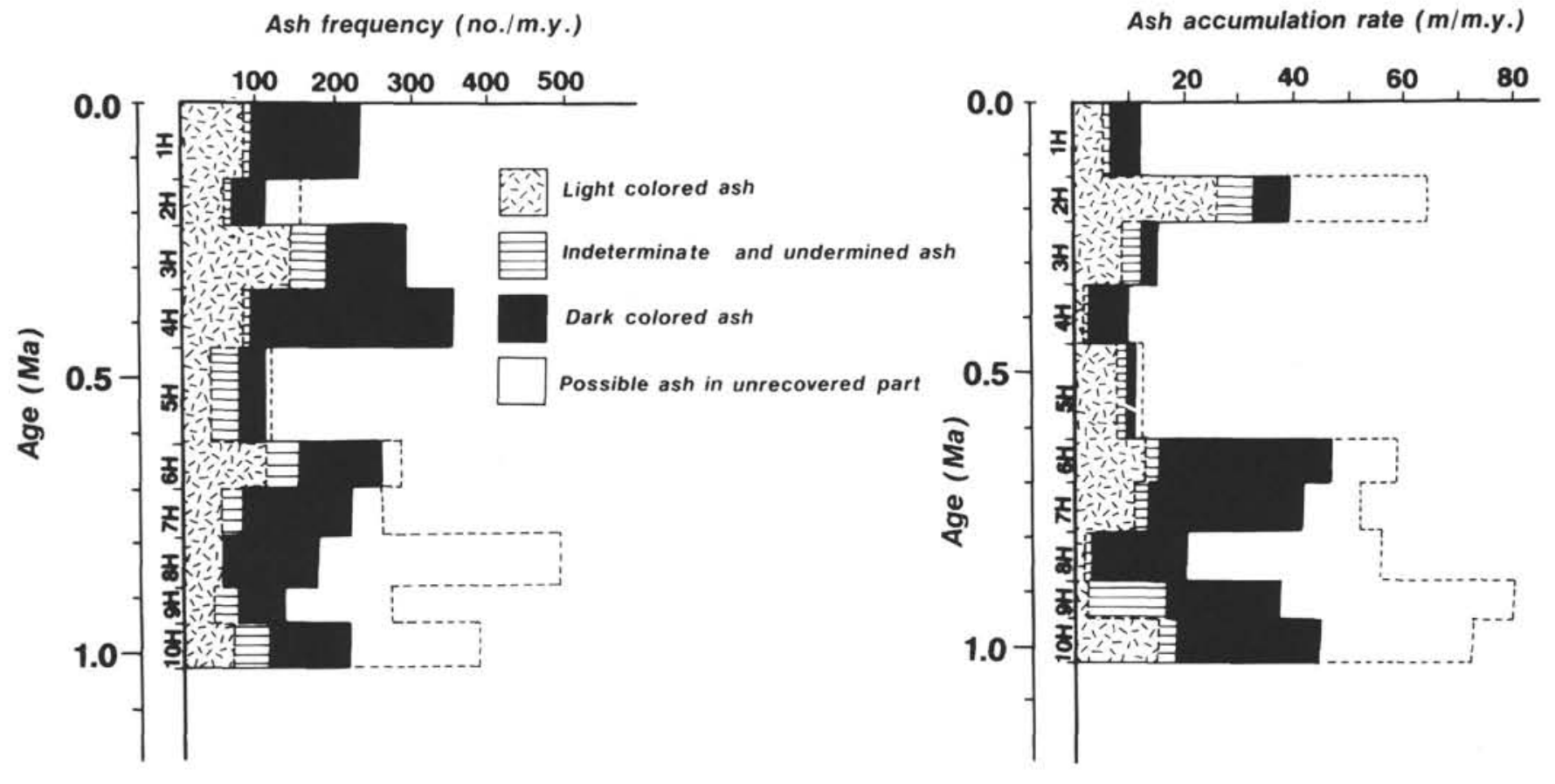

Figure 8. Accumulation rate of the Quaternary volcaniclastic materials of the forearc Site 792. A. Ash frequency indicated by ash number per million years, calculating by the constant sedimentation rates of muddy hemipelagites. B. Ash sedimentation rate obtained by the same method as in Figure $8 \mathrm{~A}$.

basalt series of that classification scheme. Yuasa (1985) compiled all the published chemical compositions of the Izu-Bonin volcanic rocks and classified them into two types: a primitive, low-alkali tholeiite series in the southern part of the arc, and a high-alkali or calc-alkalic series in the northern part. Yuasa (1985), Notsu et al. (1983), and Ikeda and Yuasa (1989) also state that the volcanic rocks are more primitive in the south compared with those in the north. The boundary between the two major types is the Sofugan Tectonic line near the Sofugan district (Yuasa, 1985; Yuasa and Murakami, 1985). Alkali olivine basalt also exists near the Iwojima Islands near the backarc side of the arc (Tsuya, 1937).

\section{$\mathrm{SiO}_{2}-\mathrm{TiO}_{2}$ Relationship}

An inverse relationship exists between $\mathrm{SiO}_{2}$ and $\mathrm{TiO}_{2}$ (Figs. 15A and 15B), as has been noted in other island-arc volcanic suites, especially in calc-alkaline andesites (Gill, 1981), reflecting early crystallization of iron-titanium oxides. Taylor and Nesbitt (1990) showed that the $\mathrm{TiO}_{2}$ contents of volcanic rocks are minimal near trench axes and increase toward the backarc.

\section{$\mathrm{SiO}_{2}-\mathrm{K}_{2} \mathrm{O}$ Relationship}

Based on $\mathrm{K}_{2} \mathrm{O}$ contents, Gill (1981) classified andesitic rocks into low-K, medium-K, and high-K series. Miyashiro $(1973,1974)$ proposed another classification for the andesitic rocks, based on their $\mathrm{SiO}_{2}$ and $\mathrm{FeO} / \mathrm{MgO}$ ratios. No definite classification has been proposed for more silicic rocks. Figure 16 shows that most of the tephra layers fall into Gill's low-K series, a few into his medium-K series, and very rare ones into his high-K series. Thus, mostly the volcanic rocks of the Izu-Bonin Arc belong to Gill's low-K series and Miyashiro's tholeiite and calc-alkalic rock series. The relative proportion of calc-alkalic to tholeiitic tephra is low compared with values in other arcs and active continental margins. However, this may be an artifact arising from the occurrence of older data published for the rocks of the northern part of the Izu-Bonin Arc. Recently, Hamuro et al. (1980, 1983), Ikeda and Yuasa (1989), and Hochstaedter and Gill (1990) published new data for submarine volcanic rocks that indicate the tephra layers of the low-K series were brought to the depositional site from the volcanic front by subaerial and subaqueous processes.

\section{DISCUSSION}

\section{Frequency of Tephra Occurrence}

The frequency pattern of the tephra layers in the forearc sites document two definite episodes of increased volcanism: in the late Eocene to early Oligocene (Leg 125 Shipboard Scientific Party, 1989) and in the Pliocene-Pleistocene. In addition, a small peak occurs in the late middle Miocene (ca. $10 \mathrm{Ma}$ ). These peaks correspond to the following regional tectonic and geologic events: before the opening of the Parece Vela Basin, after the cessation of Shikoku Basin spreading (Seno and Maruyama, 1984; Kobayashi and Nakada, 1978), and before the rifting of the Izu-Bonin backarc regions (Fujioka, 1988). The frequency of tephra layers means that the episodes of backarc spreading started just after the cessation of the intense arc volcanism. A major debate about the relationship between backarc spreading and arc volcanism has been discussed. Our results corroborate the contention (e.g., cf. Scott and Kroenke, 1980; Rodolfo, 1980) that backarc spreading started during periods of quiescence in arc volcanism. Our results also provide additional details concerning the petrology of magmas erupted during arc rifting.

The younger tephras from the forearc sites are colored and chemically bimodal; that is, they are black ash and white ash, or basaltic and rhyolitic, respectively. This bimodality increases from Pliocene to Holocene time. Similar bimodal volcanism was noted in the Basin and Range province and the Cascade region of the North America, and in volcanic rocks produced from Eocene to Holocene time, when regional extensional stress fields were predominant (Christiansen and 
A

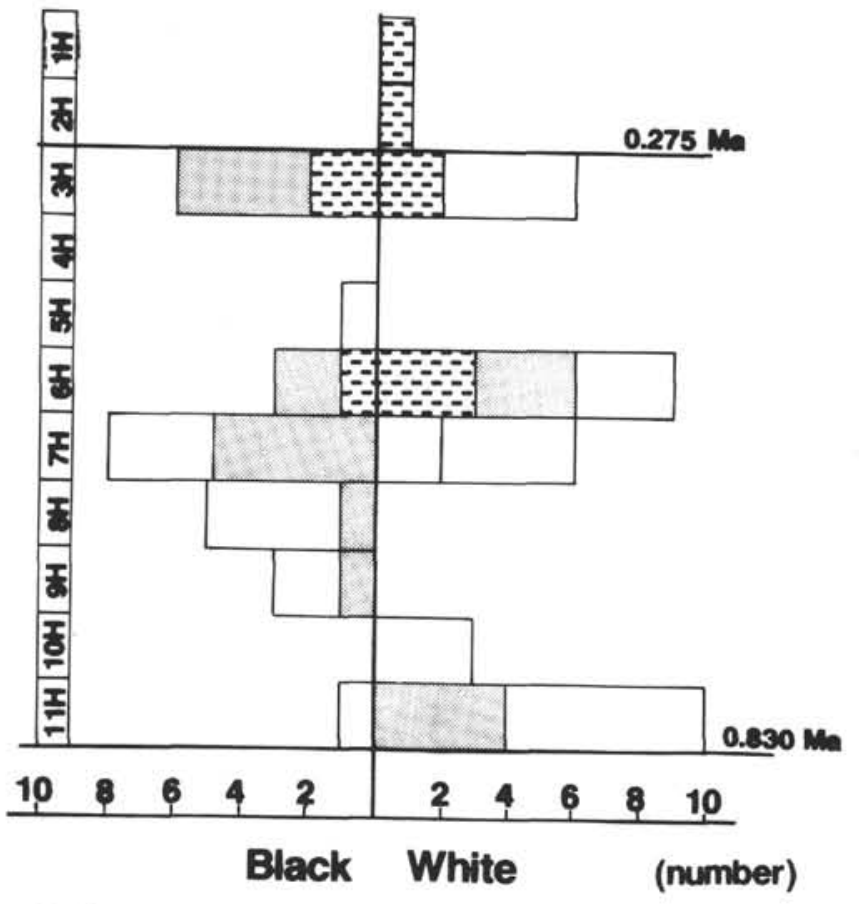

B

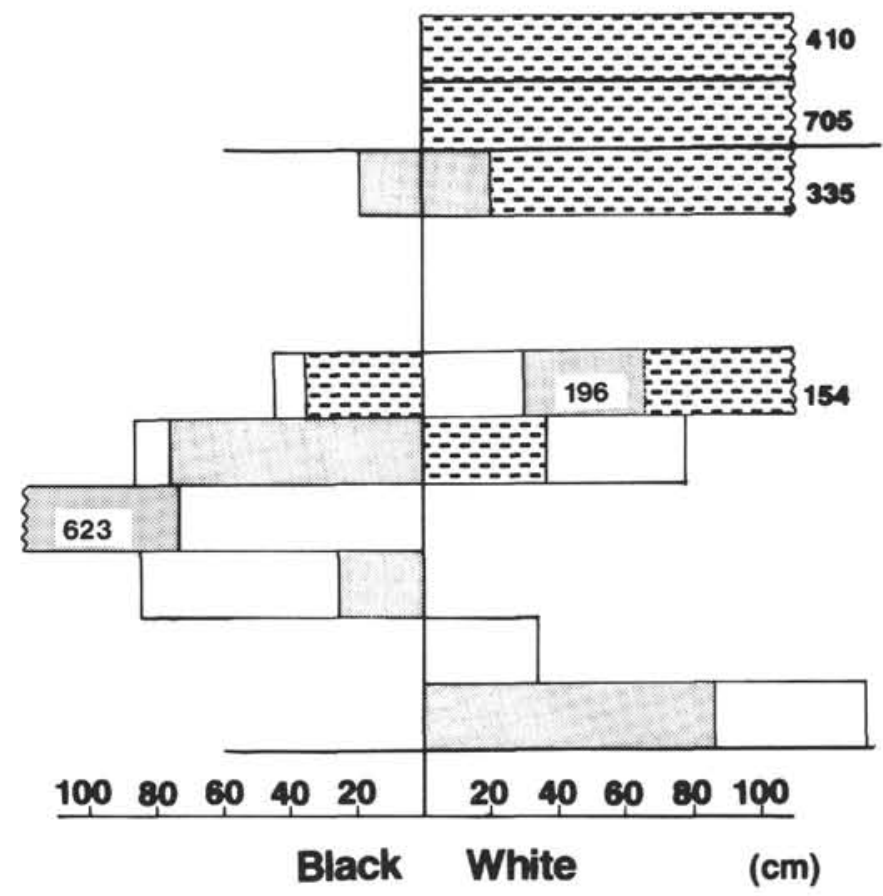

\section{Granule Size}

\section{Sand Size}

\section{Silt Size}

Figure 9. Frequency (A) and thickness (B) of the tephra layers at Site 793, Izu-Bonin forearc. The thickness and frequency of the black and white tephra layers are plotted vs. the core number.

Lipman, 1972; Eaton, 1982, 1984). Kennett et al. (1979) documented and discussed a pulse of circum-Pacific volcanism during the Quaternary period and named it the Cascadian pulse. The Izu-Bonin explosive volcanism was similar in time to the Cascadian event.

Cadet and Fujioka (1980) and Fujioka (1983a) documented that maxima of explosive volcanism in the northeast Japan (Tohoku) arc occurred during the middle Miocene and Pliocene-Pleistocene. The younger of these two maxima (ca. $3 \mathrm{Ma}$ ) is contemporaneous with that of the Izu-Bonin Arc. In the Tohoku arc, Pliocene-Pleistocene explosive volcanism was not bimodal, although the middle Miocene volcanism was (Konda, 1974; Fujioka, 1983b). We speculate that the difference of the Pliocene-Pleistocene volcanic products in the IzuBonin and Tohoku arcs may be ascribed to the difference of the crustal thickness and nature in crust of the two arcs.

\section{Origin of the Black Tephras}

The chemical composition and grain-size distribution of the black tephras at Site 792 are comparable to those produced by several basaltic eruptions in the Aogashima Islands (Takada et al., in press; Tsuya, 1937). The chemical composition of the Aogashima basalt, especially the Kurosaki volcanics (the lowermost exposure of the island, of unknown age), displays low $\mathrm{Ti}$ and $\mathrm{K}_{2} \mathrm{O}$ and high $\mathrm{CaO}$ and $\mathrm{FeO}$. Site 792 black tephras are chemically comparable to the Kurosaki volcanics (Takada et al., in press). The location of Site 792 is at the fork of the Aogashima Canyon, about $60 \mathrm{~km}$ east of Aogashima Island. The head of one branch of the canyon, South Canyon, is located immediately east of Aogashima. Some of the black tephra layers may possibly be correlated by chemistry with the Kurosaki volcanics of Aogashima Volcano. If they are correlated, basaltic tephra at Site 792 were either transported in the air as air fall or were remobilized along the canyon after air-fall deposition.

\section{$\mathrm{K}_{2} \mathrm{O}$ Contents of Volcanic Glass}

Dickinson and Hatherton (1967), Dickinson (1968), and Nielson and Stoiber (1973) demonstrated that the $\mathrm{K}_{2} \mathrm{O}$ contents of volcanic rocks bear a close relationship to the depth of the magma from which they were generated. Consequently, the $\mathrm{K}_{2} \mathrm{O}$ contents of the tephras should also reflect the depth of the magma from which the tephra layers were derived. High- $\mathrm{K}_{2} \mathrm{O}$ tephra layers increased in abundance since $3 \mathrm{Ma}$ along the Izu-Bonin Arc. If they were derived from the Izu-Bonin Arc, they might indicate gradual thickening of the arc crust since the Pliocene, consistent with Miyashiro's (1974) observation that the proportion of calc-alkalic series rocks increases as the crust thickens. This interpretation is invalid if the high $-\mathrm{K}_{2} \mathrm{O}$ ash came from another source.

\section{Possible Correlation of High-K Tephras with Widespread Regional Deposits}

The fine-grained, well-sorted, white tephra layers consisting mainly of bubble-wall glass shards may belong to other tephras than those of the Izu-Bonin Arc, widespread throughout the region, such as those described by Machida and Arai (1988) and Furuta et al. (1986). Fujioka et al. (this volume) correlated the tephras at Hole 442A in the Shikoku Basin with 
Table 3. Petrographic characteristics of tephra layers from the forearc sites.

\begin{tabular}{|c|c|c|c|c|c|c|c|c|c|c|c|c|c|c|c|c|c|c|c|c|c|}
\hline \multirow{2}{*}{\multicolumn{2}{|c|}{$\begin{array}{l}\text { Core, section, } \\
\text { Interval }(\mathrm{cm})\end{array}$}} & \multirow[t]{2}{*}{ Lithology } & \multirow{2}{*}{$\begin{array}{l}\text { Thickness } \\
\text { (cm) }\end{array}$} & \multirow{2}{*}{\multicolumn{2}{|c|}{ Grain size }} & \multirow{2}{*}{$\begin{array}{c}\operatorname{Max} g \\
(\mathrm{~mm})\end{array}$} & \multirow{2}{*}{$\begin{array}{c}\text { Max II } \\
(\mathrm{mm})\end{array}$} & \multirow{2}{*}{\multicolumn{2}{|c|}{$\begin{array}{c}\text { Mode } \\
(\mathrm{g} \mid \% / \text { cry\%/li\%) }\end{array}$}} & \multicolumn{6}{|c|}{ Constituent minerals } & \multirow[t]{2}{*}{ Glass shape } & \multicolumn{3}{|c|}{ Refractive Index } & \multirow[t]{2}{*}{ Comment } & \\
\hline & & & & & & & & & & pl 0 & $\mathrm{cpx} \mid$ & opx & ho & bi & op & & (mean) & $(\min )$ & $(\max )$ & & \\
\hline 6.792A. & & & & & & & & & & & & & & & & & & & & & \\
\hline $1 \mathrm{H}-1$. & 38.40 & M. & 8 & & v.f.sand & 0.15 & & & $90 / 10 / 0$ & + & & & & & & bw & 1.5052 & 1.5019 & 1.5071 & & \\
\hline $1 \mathrm{H} \cdot 2$ & 64.66 & M. & 28 & & v.t.sand & 0.2 & & & $90 / 10 / 0$ & + & + & & & & + & bw & 1.5107 & 1.5087 & 1.513 & br glass(t.sand) mixed & \\
\hline $1 \mathrm{H}-3$ & $138-140$ & SL & 19 & v.t.sand- & m.sand & 0.4 & 0.15 & & $90 / 8 / 2$ & + & + & & & & + & bw & 1.5115 & 1.5097 & 1.5132 & $\mathrm{bw}(\mathrm{m}$.sand;Max $0.4 \mathrm{~mm}) \& \mathrm{bw}(\mathrm{v} . \mathrm{f}$.sand $)$ & \\
\hline 1H-5, & 81.83 & M. & 3 & v.t.sand- & c.sand & 0.55 & 0.15 & & $93 / 5 / 2$ & ++ & ++ & & ++ & & ++ & bw & 1.5096 & 1.509 & 1.5108 & bf glass(m.sand) mixed & \\
\hline $1 \mathrm{H}-6$ & $119-121$ & SL & 4 & v.t, sand- & c.sand & 0.55 & 0.15 & & $90 / 8 / 2$ & + & ++ & & & & ++ & tub.pum & 1.5122 & 1.5107 & 1.5031 & & muddy \\
\hline $1 \mathrm{H}-6$ & $146-148$ & D & & v.t.sand- & c.sand & 0.2 & $>0.9$ & $\mathrm{mi}$ & $50 / 15 / 35$ & + & ++ & & & & + & bw & 1.5051 & 1.5046 & 1.5057 & & muddy \\
\hline $2 \mathrm{H}-1$. & $117-119$ & D & & & silt & 0.1 & & & $95 / 5 / 0$ & $+*$ & & & & & $+*$ & bw & & & & & \\
\hline $2 \mathrm{H}-2$ & $87 \cdot 89$ & M & 12 & v.t. sand. & m.sand & 0.35 & 0.1 & & $96 / 12 / 2$ & + & ++ & & + & & ++ & bw (fine $)>p$ um (coarse $)$ & 1.5196 & 1.5082 & 1.5211 & & \\
\hline $2 \mathrm{H}-3$ & $60-62$ & SL & 5 & vif.sand. & c.sand & 0.9 & $>0.6$ & $\mathrm{mt}$ & $50 / 20 / 30$ & $\omega+$ & ++ & & ++ & & & br.cry.bw & 1.5204 & 1.516 & 1.5285 & & \\
\hline $2 \mathrm{H}-4$ & $18-20$ & D & D & v.t.sand. & t. sand & 0.2 & 0.15 & & $80 / 10 / 10$ & ++ & ++ & & & & + & br.cry>pum & & & & & \\
\hline $2 \mathrm{H}-4$ & $23-25$ & D & D & v.t.sand. & 1. sand & 0.35 & 0.15 & & $91 / 8 / 1$ & ++ & ++ & & & & & bw & 1.5179 & 1.5156 & 1.5195 & br glass mixed & \\
\hline $3 \mathrm{H}-1$, & 50.52 & SL & 2 & v.t. sand. & c.sand & 0.9 & 0.15 & & $40 / 30 / 30$ & +++ & + & & & & + & pum,tub, br & & & & & \\
\hline $3 \mathrm{H}-1$, & $86-88$ & ML & 14 & v.t.sand & t. sand & 0.25 & & & $95 / 5 / 0$ & ++ & & & + & & & bw & 1.5067 & 1.5058 & 1.508 & & \\
\hline $3 \mathrm{H}-1$, & $122 \cdot 124$ & M & 5 & & v.t.sand & 0.15 & 0.1 & & $95 / 4 / 1$ & + & + & & + & & ++ & bw & 1.5016 & 1.5012 & 1.502 & & \\
\hline $3 \mathrm{H}-2$ & $83-85$ & s & 3 & v.t. sand- & t. sand & 0.25 & 0.1 & & $94 / 15 / 1$ & ++ & + & + & + & & + & bw & 1.5044 & 1.5019 & 1.5061 & & \\
\hline $3 \mathrm{H} \cdot 5$ & 78.80 & s & 1. & v.t.sand & m.sand & 0.35 & 0.4 & & $30 / 30 / 40$ & +++ & ++ & ++ & & & ++ & cry,tub,bw & & & & br glass \& clear glass mixed & muddy \\
\hline $3 \mathrm{H} \cdot \mathrm{CC}$ & $(5-7)$ & $\mathrm{s}$ & 14 & & v.t.sand & 0.15 & & & $95 / 5 / 0$ & + & 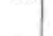 & & & & + & bw & 1.5084 & 1.5069 & 1.5091 & & \\
\hline $4 \mathrm{H}-1$, & $117-119$ & $\mathrm{~s}$ & 5 & $v$, f. sand & t. sand & 0.3 & 0.1 & & $65 / 15 / 20$ & ++ & + & + & + & & ++ & bw,br,pum & 1.5095 & 1.5087 & 1.5103 & & muddy \\
\hline $4 \mathrm{H}-2$, & $53-55$ & D & D & v.t.sand. & m.sand & 0.4 & $>0.5$ & $\mathrm{mt}$ & $45 / 15 / 40$ & ++ & ++ & ++ & & & ++ & br.tub.pum & & & & & muddy \\
\hline $4 \mathrm{H}-3$ & $20-22$ & D & D & & v.t.sand & 0.15 & & & $90 / 10 / 0$ & ++ & ++ & & & & ++ & bw & & & & & muddy \\
\hline $4 \mathrm{H}-3$ & $101-103$ & $\mathrm{~s}$ & 4 & & v.t.sand & 0.2 & 0.2 & & $45 / 15 / 40$ & ++ & ++ & & + & + & ++ & br.cry.bw & & & & & \\
\hline $4 \mathrm{H}-4$ & $96-98$ & M. & 9 & & $v$.t.sand & 0.15 & & & $95 / 5 / 0$ & ++ & & & & & ++ & bw & 1.516 & 1.5148 & 1.5168 & & \\
\hline $4 \mathrm{H}-5$, & $19-21$ & D & D & v.t.sand- & c.sand & 0.4 & 0.4 & & $50 / 20 / 30$ & ++ & + & & + & + & + & bw.br & & & & br glass\&bw glass mixed & muddy \\
\hline $4 \mathrm{H}-5$, & 57.59 & M. & 15 & v.t.sand & c.sand & 0.4 & 0.1 & $\mathrm{mt}$ & $65 / 15 / 20$ & + & ++ & ++ & & & + & pum,bw & 1.5309 & 1.5195 & 1.533 & & \\
\hline $4 \mathrm{H}-6$, & $48-50$ & SL & 2 & fsand . & m.sand & 0.4 & 0.3 & $\mathrm{mt}$ & $20 / 20 / 60$ & +++ & + & + & & & ++ & cry.sc & & & & & muddy \\
\hline $4 \mathrm{H}-7$. & $11-13$ & SL & 1 & v.f.sand- & t. sand & 0.4 & 0.15 & & $80 / 10 / 10$ & + & + & & + & & + & bw>pum.br & 1.503 & 1.5021 & 1.504 & pum. cry(m.sand)\&bw(v.f.sand)mixed & muddy \\
\hline $5 \mathrm{H}-1$ & 37.39 & M. & 5 & v.f.sand- & t. sand & 0.3 & & & 95/ $5 / 0$ & ++ & & & & & & bw & 1.507 & 1.5048 & 1.5098 & & muddy \\
\hline $5 \mathrm{H}-2$ & $45-47$ & SL & 4 & & v.t.sand & 0.15 & & & $95 / 5 / 0$ & + & + & & + & & & bw & 1.5105 & 1.5087 & 1.5112 & & \\
\hline $5 \mathrm{H}-2$ & $141-143$ & SL & 3 & v.f.sand- & c.sand & $>0.1$ & $>0.1$ & $\mathrm{mt}$ & $60 / 10 / 30$ & $+t$ & & & & & & cry,br>tub.ow & & & & & muddy \\
\hline $5 \mathrm{H}-3$ & 57.59 & M. & 7 & & v.t.sand & 0.08 & & & $95 / 5 / 0$ & ++ & & & & & & bw & 1.5145 & 1.5122 & 1.5156 & & \\
\hline $5 \mathrm{H}-3$ & $131-133$ & M. & 90 & v.t. sand- & t. sand & 0.2 & & & $95 / 5 / 0$ & + & + & & + & & + & bw & 1.5013 & 1.4998 & 1.5024 & tub(c.sand) mixed & muddy \\
\hline $6 \mathrm{H}-2$ & $17 \cdot 19$ & M. & 18 & 1. sand. & m.sand & 0.4 & & & $95 / 5 / 0$ & + & & & & & & bw & 1.5046 & 1.5042 & 1.5053 & bw.pum\&br glass mixed & \\
\hline $6 \mathrm{H}-2$ & 44.46 & M & 7 & t. sand & c.sand & 0.15 & $>0.6$ & $m t$ & $20 / 50 / 30$ & +++ & ++ & + & & & & cry & & & & Max crystal $0.7 \mathrm{~mm}$ & \\
\hline $6 \mathrm{H}-3$ & $130 \cdot 132$ & M. & 22 & i.sand - & m.sand & 0.7 & $>0.7$ & $\mathrm{mt}$ & $40 / 40 / 20$ & +++ & ++ & ++ & & & & br,cry,pum,tub & & & & & \\
\hline $6 \mathrm{H}-4$ & 97.99 & M. & 11 & v.t.sand- & t. sand & 0.15 & & & $95 / 5 / 0$ & + & 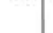 & & & & 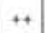 & bw & 1.5078 & 1.5067 & 1.5084 & & \\
\hline $7 \mathrm{H}-2$ & $20-21$ & $P$ & 2 & & silt & 0.15 & & & 95. $5 / 0$ & + & & & & & + & bw & & & & very fine & \\
\hline $7 \mathrm{H}-2$ & $48-50$ & SL. & 11 & v.t.sand. & t.sand & 0.2 & & & $95 / 5 / 0$ & ++ & & & & & ++ & bw & 1.5118 & 1.5106 & 1.513 & br $(0.5 \mathrm{~mm})$ mixed & \\
\hline $7 \mathrm{H}-3$ & 81.83 & M. & 5 & v.f.sand. & f. sand & 0.2 & 0.08 & & & + & ++ & & & & + & $b r, b w, c r y$ & 1.5082 & 1.5065 & 1.5093 & & \\
\hline $7 \mathrm{H} \cdot 3$ & $110-112$ & SL & 72 & v.t.sand. & t. sand & 0.2 & 0.1 & & $90 / 8 / 2$ & + & ++ & & & & ++ & $b w>b r$ & 1.509 & 1.5078 & 1.5099 & br glass $(0.2 \mathrm{~mm})$ mixed & 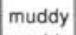 \\
\hline $7 \mathrm{H}-4$ & $103-104$ & M. & 5 & 1. sand - & c.sand & 0.4 & 0.3 & $\mathrm{mt}$ & $60 / 15 / 20$ & ++ & ++ & & & & & br,cry & & & & & muddy \\
\hline $8 \mathrm{H}-2$ & $64-66$ & D & & v.f.sand- & m.sand & 0.3 & 0.1 & & $90 / 9 / 1$ & + & 1 & & + & & + & bw & 1.5031 & 1.5026 & 1.5038 & & \\
\hline $8 \mathrm{H}-3$ & 40.42 & M. & 70 & v.t.sand- & f.sand & 0.2 & 0.7 & & $91 / 8 / 1$ & ++ & ++ & + & + & & ++ & bw & 1.4996 & 1.4989 & 1.5003 & br glass $(0.2 \mathrm{~mm})$ mixed & muddy \\
\hline $9 \mathrm{H}-4$ & 6.8 & SL & 93 & v.f.sand- & $\mathrm{m}$.sand & 0.5 & 0.1 & & $87 / 10 / 3$ & ++ & ++ & & ++ & & ++ & bw>tub,pum.cry,br & 1.5209 & 1.5056 & 1.5347 & tub, pum(Max 0.5mm),br\&bw mixed & muddy \\
\hline $10 \mathrm{H}-2$ & 6.8 & SL & 2 & v.t.sand- & $\mathrm{m}$.sand & 0.3 & 0.05 & & 91/ $8 / 1$ & ++ & ++ & & ++ & & & bw.pum>br & 1.5206 & 1.5197 & 1.5218 & br glass mixed & \\
\hline$=0$ & & & & & & & & & & & & & & & & & & & & & \\
\hline
\end{tabular}


Table 3 (continued).

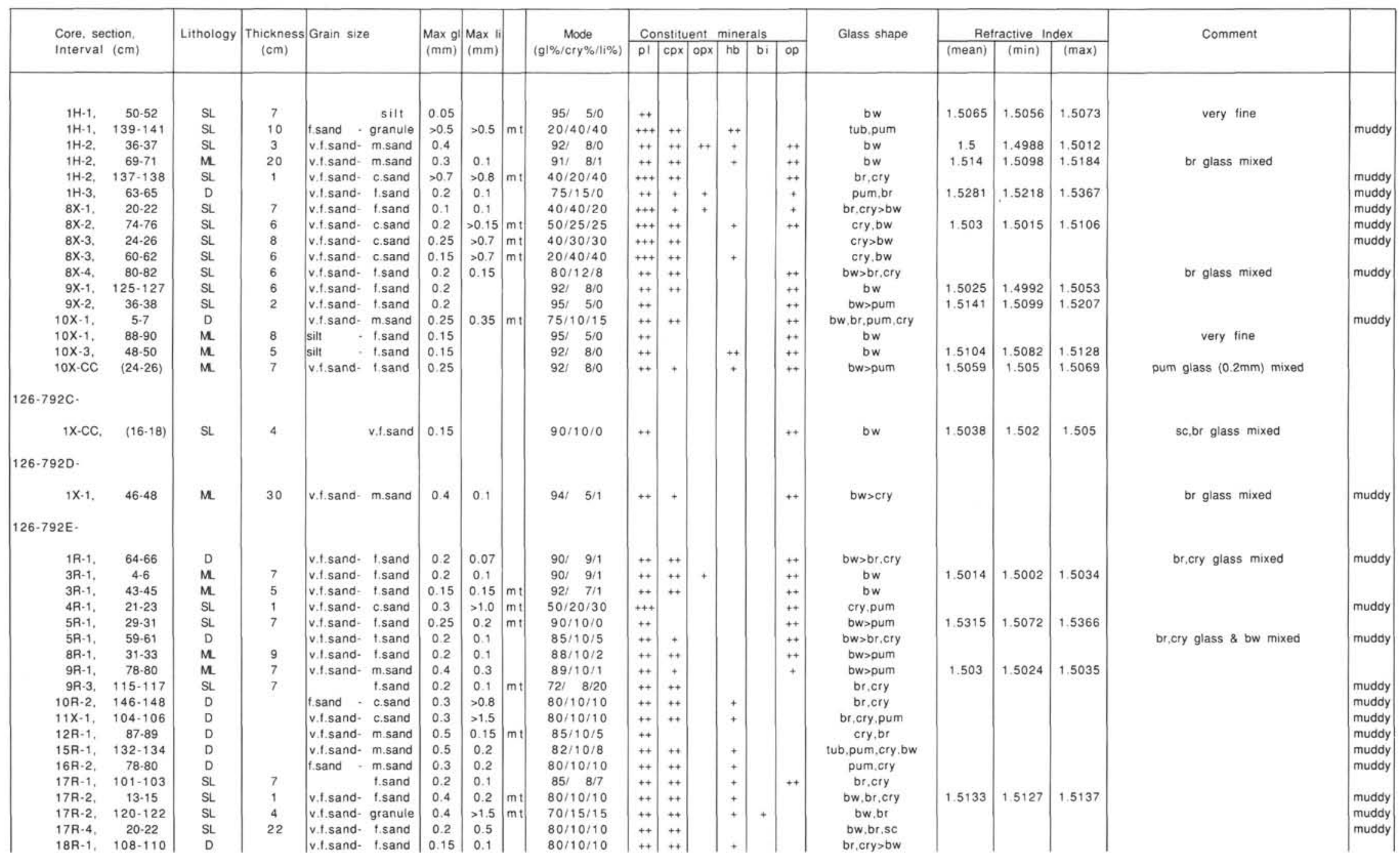


Table 3 (continued).

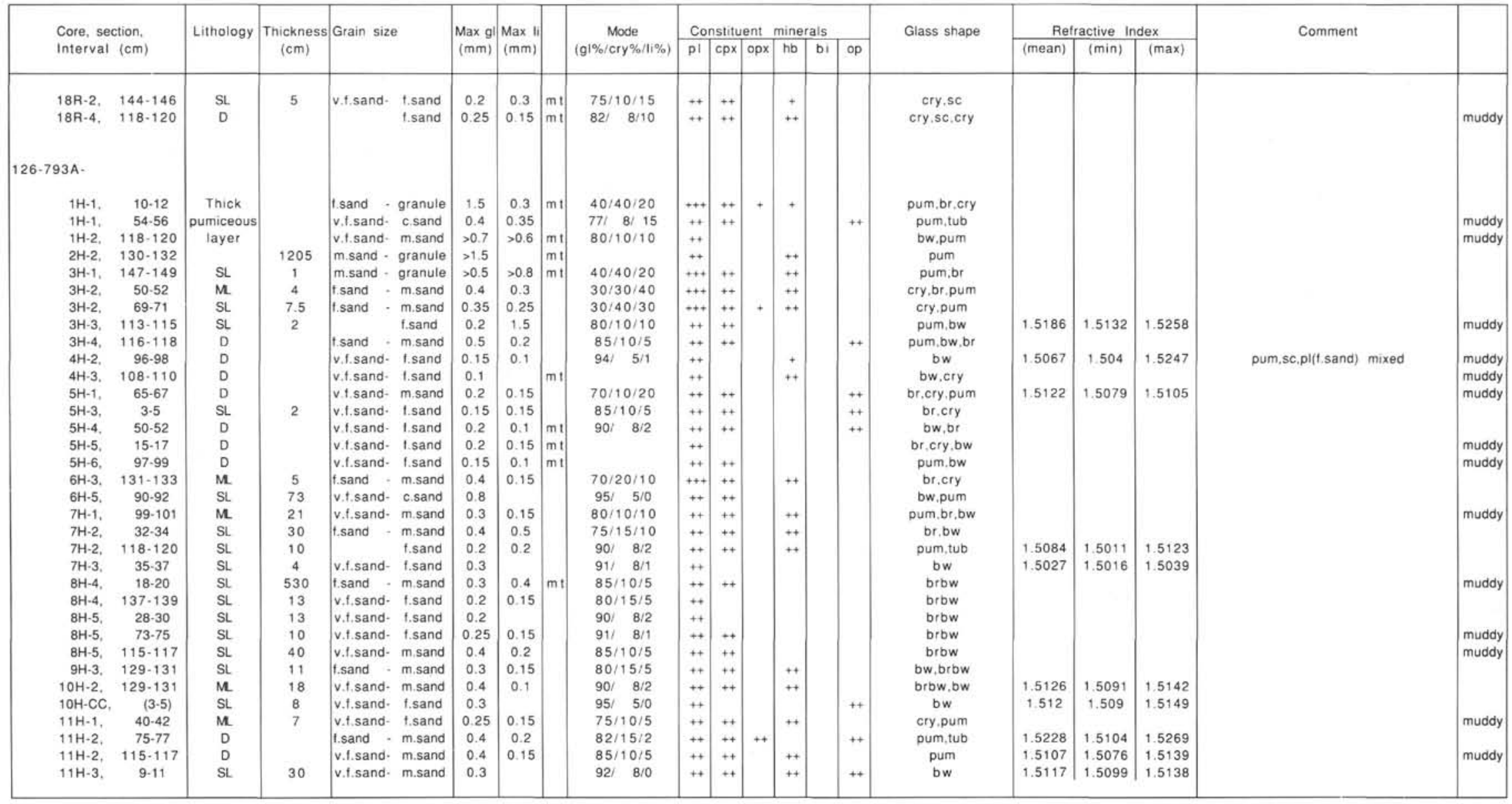

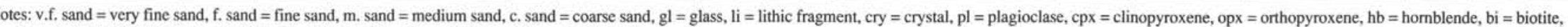

op. $=$ opaque mineral, pum. $=$ pumice, $\mathrm{br}=$ brown glass, tub $=$ tubular, and $\mathrm{bw}=$ bubble wall. 
Table 4. Catalog of the ash intervals in Hole 782A (tephra layers), Leg 125, in the Izu-Bonin forearc high.

\begin{tabular}{|c|c|c|}
\hline $\begin{array}{l}\text { Core, section, } \\
\text { interval }(\mathrm{cm})\end{array}$ & $\begin{array}{l}\text { Depth } \\
\text { (mbsf) }\end{array}$ & $\begin{array}{c}\text { Volcanic } \\
\text { layer }\end{array}$ \\
\hline \multicolumn{3}{|l|}{$125-782 \mathrm{~A}$} \\
\hline $1 \mathrm{H}-1,90$ & 0.9 & 1 \\
\hline $1 \mathrm{H}-3,24$ & 3.24 & 2 \\
\hline $1 \mathrm{H}-3,130$ & 4.3 & 3 \\
\hline $2 \mathrm{H}-4,86$ & 15.16 & 4 \\
\hline $2 \mathrm{H}-4,90$ & 15.2 & 5 \\
\hline $2 \mathrm{H}-4,103-104$ & 15.33 & 6 \\
\hline $2 \mathrm{H}-4,114$ & 15.44 & 7 \\
\hline $2 \mathrm{H}-4,131$ & 15.61 & 8 \\
\hline $2 \mathrm{H}-5,107-115$ & $16.87-16.95$ & 9 \\
\hline $2 \mathrm{H}-5,127-150$ & 17.3 & 10 \\
\hline $2 \mathrm{H}-6,0-8$ & 18.1 & 11 \\
\hline $2 \mathrm{H}-6,85-86$ & 18.16 & 12 \\
\hline $5 \mathrm{H}-6,18-19$ & 45.98 & 13 \\
\hline $6 \mathrm{H}-3,21-27$ & $51.01-51.07$ & 14 \\
\hline $6 \mathrm{H}-4,84-91$ & $53.14-53.21$ & 15 \\
\hline $6 \mathrm{H}-655-57$ & $55.85-55.87$ & 16 \\
\hline $6 \mathrm{H}-6,87-90$ & $56.17-56.20$ & 17 \\
\hline $7 \mathrm{H}-3,67-69$ & $60.97-60.98$ & 18 \\
\hline $7 \mathrm{H}-3,106-110$ & $61.36-61.40$ & 19 \\
\hline $7 \mathrm{H}-4,79-84$ & $62.53-62.64$ & 20 \\
\hline $7 \mathrm{H}-7,59-61$ & $66.89-66.91$ & 21 \\
\hline $8 \mathrm{H}-4,94-95$ & 72.24 & 22 \\
\hline $8 \mathrm{H}-4,122-123$ & 72.52 & 23 \\
\hline $11 X-1,54-57$ & $96.24-96.27$ & 24 \\
\hline $11 X-1,110-117$ & $96.80-96.87$ & 25 \\
\hline $11 X-2,37-38$ & 97.57 & 26 \\
\hline $11 X-3,0-1$ & 98.7 & 27 \\
\hline $11 X-3,9.5-10$ & 98.8 & 28 \\
\hline $11 X-3,51-54$ & $99.21-99.24$ & 29 \\
\hline $12 \mathrm{X}-1,20$ & 105.5 & 30 \\
\hline $12 X-1,28-29$ & 105.58 & 31 \\
\hline $12 X-2,9-10$ & 106.8 & 32 \\
\hline $12 \mathrm{X}-2,16-17$ & 105.96 & 33 \\
\hline $12 \mathrm{X}-\mathrm{CC}, 22-23$ & 110.27 & 34 \\
\hline $13 \times-2,103-104$ & 117.53 & 35 \\
\hline $13 X-3,51.5-52.5$ & 118.52 & 36 \\
\hline $14 X-2,10-11$ & 126.2 & 37 \\
\hline $14 X-2,82-83$ & 126.52 & 38 \\
\hline $14 X-2,112-116$ & $117.22-127.26$ & 39 \\
\hline $14 X-2,140-144$ & $127.50-127.54$ & 40 \\
\hline $14 X-3,41-45$ & $128.01-128.05$ & 41 \\
\hline $14 X-5,15-18$ & $130.0-130.65$ & 42 \\
\hline $\begin{array}{l}15 X-2, \\
105.5-106.5\end{array}$ & 136.86 & 43 \\
\hline $15 X-2,115$ & 136.95 & 44 \\
\hline $15 \times-2,117$ & 136.97 & 45 \\
\hline $15 X-3,8-16$ & $137.38-137.54$ & 46 \\
\hline $15 X-3,101-105$ & $138.31-138.35$ & 47 \\
\hline $15 X-3,113-115$ & $138.41-138.43$ & 48 \\
\hline $15 X-4,13.5-17.0$ & $138.93-138.97$ & 49 \\
\hline $16 \times-3,96-97$ & 147.86 & 50 \\
\hline $17 X-1,54-55$ & 154.14 & 51 \\
\hline $17 \times-2,3-5$ & $155.13-155.15$ & 52 \\
\hline $17 X-2,102-103$ & 156.12 & 53 \\
\hline $17 X-2,124-125$ & 156.34 & 54 \\
\hline $17 X-3,121$ & 157.81 & 55 \\
\hline $17 X-4,0-2$ & $158.08-158.12$ & 56 \\
\hline $17 X-4,73-74$ & 158.83 & 57 \\
\hline
\end{tabular}

Table 4 (continued).

\begin{tabular}{|c|c|c|}
\hline $\begin{array}{l}\text { Core, section, } \\
\text { interval }(\mathrm{cm})\end{array}$ & $\begin{array}{l}\text { Depth } \\
\text { (mbst) }\end{array}$ & $\begin{array}{c}\text { Volcanic } \\
\text { layer }\end{array}$ \\
\hline $17 X-5,139-141$ & $160.99-161.01$ & 58 \\
\hline $18 X-1,10-16$ & $163.36-163.38$ & 59 \\
\hline $19 X-1,147-148$ & 174.37 & 60 \\
\hline $19 \times-2,23-27$ & $174.63-174.67$ & 61 \\
\hline $19 X-3,104-105$ & 176.94 & 62 \\
\hline $19 \mathrm{X}-\mathrm{CC}, 13-14$ & 178.08 & 63 \\
\hline $20 \mathrm{X} \cdot \mathrm{CC}, 10$ & 183.26 & 64 \\
\hline $21 X-1,51-53$ & $192.61-192.62$ & 65 \\
\hline $21 X-1.64-66$ & $192.74-192.76$ & 66 \\
\hline $21 X-2.48-51$ & $194.08-194.10$ & 67 \\
\hline $21 X \cdot 2,82-86.5$ & $194.42-194.46$ & 68 \\
\hline $21 X-2,96-97$ & 194.56 & 69 \\
\hline $21 X-2,127-128$ & 194.87 & 70 \\
\hline $21 X-3,0-1$ & 195.1 & 71 \\
\hline $21 X-3,72$ & 195.82 & 72 \\
\hline $21 \times-5,42-45$ & $198.52-198.55$ & 73 \\
\hline $23 \times-2,15-16$ & 213.15 & 74 \\
\hline $23 X-3,23-26$ & $214.73-214.76$ & 75 \\
\hline $23 X-3,93-94$ & 215.33 & 76 \\
\hline $23 X-4,107-109$ & $217.07-217.09$ & 77 \\
\hline $23 \times-4,132-133$ & 217.32 & 78 \\
\hline $23 x-5,40-45$ & $217.90-217.95$ & 79 \\
\hline $23 X-5,83-86$ & $218.33-218.36$ & 80 \\
\hline $23 \times-6,67-63$ & 219.67 & 81 \\
\hline $23 X-6,134-137$ & $220.34-220.37$ & 82 \\
\hline $23 X-6,141-144$ & $220.41-220.44$ & 83 \\
\hline $24 X-1,44-45$ & 221.44 & 84 \\
\hline $24 X-2,2-3$ & 222.7 & 85 \\
\hline $25 \times-4,32-36$ & $235.52-235.56$ & 86 \\
\hline $25 \times-4,114$ & 236.34 & 87 \\
\hline $26 \mathrm{X}-1,138$ & 241.68 & 88 \\
\hline $26 \mathrm{X}-1,140$ & 241.7 & 89 \\
\hline $26 \mathrm{X}-2,83-85$ & $242.63-242.65$ & 90 \\
\hline $28 X-1,98-99$ & 260.58 & 91 \\
\hline $28 \mathrm{X}-3,17$ & 262.77 & 92 \\
\hline $29 \times-3,9-10$ & 273.1 & 93 \\
\hline $29 \mathrm{X}-3,43-46$ & $272.63-272.66$ & 94 \\
\hline $29 X-3,90$ & 273.1 & 95 \\
\hline $29 \mathrm{X}-4,85-90$ & $274.55-274.67$ & 96 \\
\hline $29 X-5,12-15$ & $275.32-272.35$ & 97 \\
\hline $29 X-6,19-21$ & $276.89-276.91$ & 98 \\
\hline $29 \times-6,30-41$ & $277.00-277.11$ & 99 \\
\hline $29 \times-7,35-37$ & $278.55-278.57$ & 100 \\
\hline $30 \times-2,9-10$ & 280.39 & 101 \\
\hline $30 X-2,30-31$ & 280.6 & 102 \\
\hline $31 X-1,17$ & 288.67 & 103 \\
\hline $31 X-1,22-24$ & $288.72-288.74$ & 104 \\
\hline $32 \times-2,41-46$ & $295.91-295.96$ & 105 \\
\hline $32 X-4,124$ & 299.74 & 106 \\
\hline $32 X-6,32-35$ & $301.82-301.85$ & 107 \\
\hline $33 X-3,82-85$ & $307.82-307.35$ & 108 \\
\hline $33 X-5,87-88$ & 310.37 & 109 \\
\hline $33 \times-7,27-25.5$ & $312.77-312.80$ & 110 \\
\hline $34 \mathrm{X} \cdot \mathrm{CC}, 20-26.5$ & $314.79-314.85$ & 111 \\
\hline $37 X-5,85-87$ & $348.45-348.47$ & 112 \\
\hline $39 \times-2,9$ & 362.49 & 113 \\
\hline $39 \times-2,44-45.5$ & 362.88 & 114 \\
\hline $39 \times-2,70-71$ & 363.1 & 115 \\
\hline $41 \times-4,23-24$ & 384.93 & 116 \\
\hline
\end{tabular}

Note: Data from Fryer, Pearce, Stokking, et al. (1990). 
Table 5. Catalog of the ash intervals in Hole 786A (volcaniclastic layers), Leg 125, in the Izu-Bonin forearc high.

\begin{tabular}{|c|c|c|c|}
\hline $\begin{array}{l}\text { Core, section, } \\
\text { interval }(\mathrm{cm})\end{array}$ & $\begin{array}{c}\text { Depth of } \\
\text { top of } \\
\text { section } \\
\text { (mbsf) }\end{array}$ & $\begin{array}{l}\text { True depth } \\
\text { (mbsf) }\end{array}$ & $\begin{array}{c}\text { Volcaniclastic } \\
\text { layer }\end{array}$ \\
\hline \multicolumn{4}{|l|}{$125-786 \mathrm{~A}-$} \\
\hline $1 \mathrm{H}-1,62-77$ & 0 & $0.62-0.77$ & 1 \\
\hline $1 \mathrm{H}-2,3-5$ & 1.5 & $1.53-1.55$ & 2 \\
\hline $1 \mathrm{H}-2,5-18$ & 1.5 & $1.55-1.68$ & 3 \\
\hline $1 \mathrm{H}-4,12-16$ & 4.5 & $4.62-4.66$ & 4 \\
\hline $1 \mathrm{H}-4,23.5-25$ & 4.5 & $4.735-4.75$ & 5 \\
\hline $1 \mathrm{H}-5,62-111$ & 6 & $6.62-7.11$ & 6 \\
\hline $1 \mathrm{H}-6,77-78$ & 7.5 & $8.27-8.28$ & 7 \\
\hline $1 \mathrm{H}-7,5-8$ & 9 & $9.05-9.08$ & 8 \\
\hline $2 \mathrm{H}-1,10-14$ & 9.7 & $9.80-9.94$ & 9 \\
\hline $2 \mathrm{H}-1,29-30$ & 9.7 & $9.99-10.0$ & 10 \\
\hline $2 \mathrm{H}-1,80$ & 9.7 & 10.5 & 11 \\
\hline $2 \mathrm{H}-2,99-113$ & 11.2 & $12.19-12.33$ & 12 \\
\hline $2 \mathrm{H}-2,143-146$ & 11.2 & $12.63-12.66$ & 13 \\
\hline $2 \mathrm{H}-3,145-146$ & 12.7 & $14.10-14.14$ & 14 \\
\hline $2 \mathrm{H}-4,36-40$ & 14.2 & $14.56-14.60$ & 15 \\
\hline $2 \mathrm{H}-4,84-85$ & 14.2 & $15.04-15.05$ & 16 \\
\hline $2 \mathrm{H}-4,93-96$ & 14.2 & $15.13-15.16$ & 17 \\
\hline $2 \mathrm{H}-4,141-145$ & 14.2 & $15.60-15.65$ & 18 \\
\hline $2 \mathrm{H}-5,28-30$ & 15.7 & $15.98-16.00$ & 19 \\
\hline $2 \mathrm{H}-5,122-125$ & 15.7 & $16.92-16.95$ & 20 \\
\hline $2 \mathrm{H}-6,14-17$ & 17.2 & $17.34-17.37$ & 21 \\
\hline $3 \mathrm{H}-1,41-44$ & 19.2 & $19.61-19.64$ & 22 \\
\hline $3 \mathrm{H}-1,54-58$ & 19.2 & $19.74-19.78$ & 23 \\
\hline $3 \mathrm{H}-1,87-90$ & 19.2 & $20.07-20.10$ & 24 \\
\hline $3 \mathrm{H}-2,100-103$ & 20.7 & $21.70-21.73$ & 25 \\
\hline $3 \mathrm{H}-3,116-120$ & 22.2 & $23.36-23.40$ & 26 \\
\hline $3 \mathrm{H}-3,116-120$ & 22.2 & $23.36-23.40$ & 26 \\
\hline $3 \mathrm{H}-5,79-80.5$ & 25.2 & $25.99-26.005$ & 27 \\
\hline $3 \mathrm{H}-6,102-112$ & 26.7 & $27.72-27.82$ & 28 \\
\hline $3 \mathrm{H}-\mathrm{CC}, 10-17$ & 28.89 & $28.99-29.06$ & 29 \\
\hline $3 \mathrm{H}-\mathrm{CC}, 21$ & 28.89 & 29.1 & 30 \\
\hline $4 X-1,60-61$ & 28.7 & $29.30-29.31$ & 31 \\
\hline $5 X-1,39-41$ & 38.2 & $38.59-38.61$ & 32 \\
\hline $5 X-1,74-76$ & 38.2 & $38.94-38.96$ & 33 \\
\hline $5 X-1,74-76$ & 38.2 & $38.94-38.96$ & 33 \\
\hline $5 \times \cdot 1,78-79$ & 38.2 & $38.98-38.99$ & 34 \\
\hline $5 X-1,106-107$ & 38.2 & $39.26-39.27$ & 35 \\
\hline $5 X-2,14-15$ & 39.7 & $39.84-39.85$ & 36 \\
\hline $6 \mathrm{X}-1,21-25$ & 47.6 & $47.81-47.85$ & 37 \\
\hline $6 \mathrm{X}-1,93-94$ & 47.6 & $48.53-48.54$ & 38 \\
\hline $6 X-2,37-46$ & 49.1 & $49.91-49.92$ & 39 \\
\hline $6 \mathrm{X}-2,102-103$ & 49.1 & $50.12-50.13$ & 40 \\
\hline $6 X-3,126.5-128$ & 50.6 & $51.865-51.88$ & 41 \\
\hline $6 X-5,8-9$ & 53.6 & $53.68-53.69$ & 42 \\
\hline $6 X-5,22-27$ & 53.6 & $53.82-53.87$ & 43 \\
\hline $6 X-5,36-40$ & 53.6 & $53.98-54.0$ & 44 \\
\hline $7 X-1,138-140$ & 57.1 & $58.48-58.50$ & 45 \\
\hline $7 X-2,125-127$ & 58.6 & $59.85-59.87$ & 46 \\
\hline $7 X-3,3-4$ & 60.1 & $60.13-60.14$ & 47 \\
\hline $7 X-3,15.5-16$ & 60.1 & $60.255-60.26$ & 48 \\
\hline $7 X-5,9-10$ & 63.1 & $63.19-63.20$ & 49 \\
\hline $7 X-5,14-15$ & 63.1 & $63.24-63.25$ & 50 \\
\hline $7 X-5,18-24$ & 63.1 & $63.28-63.34$ & 51 \\
\hline $7 X-5,6-4-66$ & 63.1 & $63.74-63.76$ & 52 \\
\hline $7 X-6,27-28$ & 64.29 & $64.56-64.57$ & 53 \\
\hline $9 \times-3,43-44$ & 79.8 & $80.23-80.24$ & 54 \\
\hline $9 \times-3,85-89$ & 79.8 & $80.65-80.69$ & 55 \\
\hline $9 X-3,128-131$ & 79.8 & $81.08-81.11$ & 56 \\
\hline $9 X-4,23-26$ & 81.3 & $81.53-81.56$ & 57 \\
\hline $9 X-5,56-66$ & 82.8 & $83.36-83.46$ & 58 \\
\hline
\end{tabular}

Note: Data from Fryer, Pearce, Stokking, et al. (1990).

those in the Izu-Bonin forearc. Of these, tephras having high-K contents may have come from sources far to the west (e.g., from the Ryukyu Arc), carried by the prevailing westerly winds around Japan (Horn et al., 1969; Kennett, 1981). The volcanic history of the Ryukyu Arc is not well known, however, as its stratigraphy has been studied by only a few workers (Konishi, 1965; Furukawa and Isezaki, in press; Fujioka et al.,

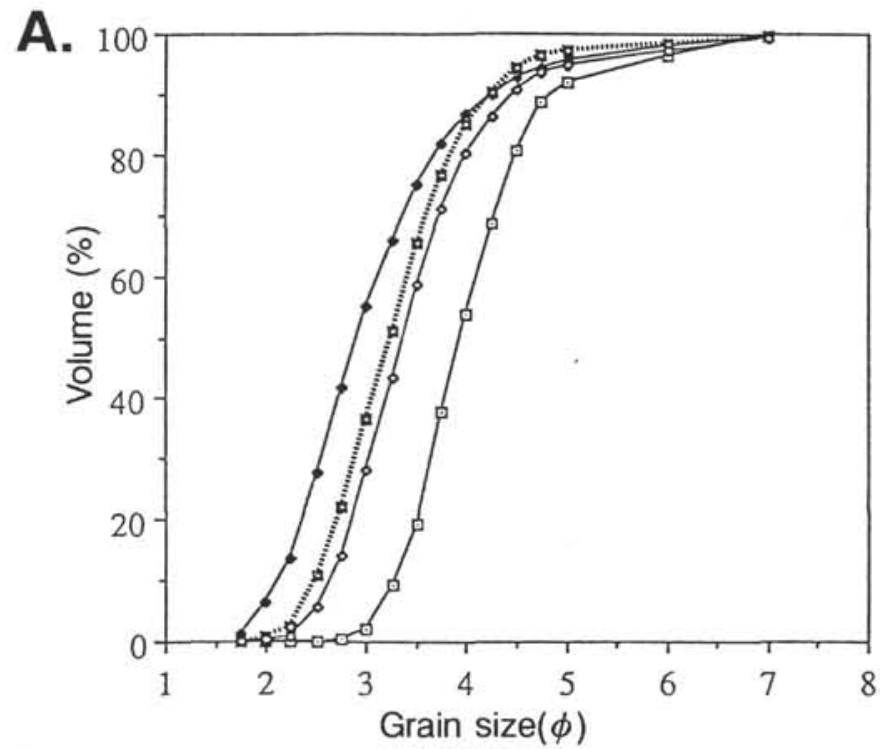

B.

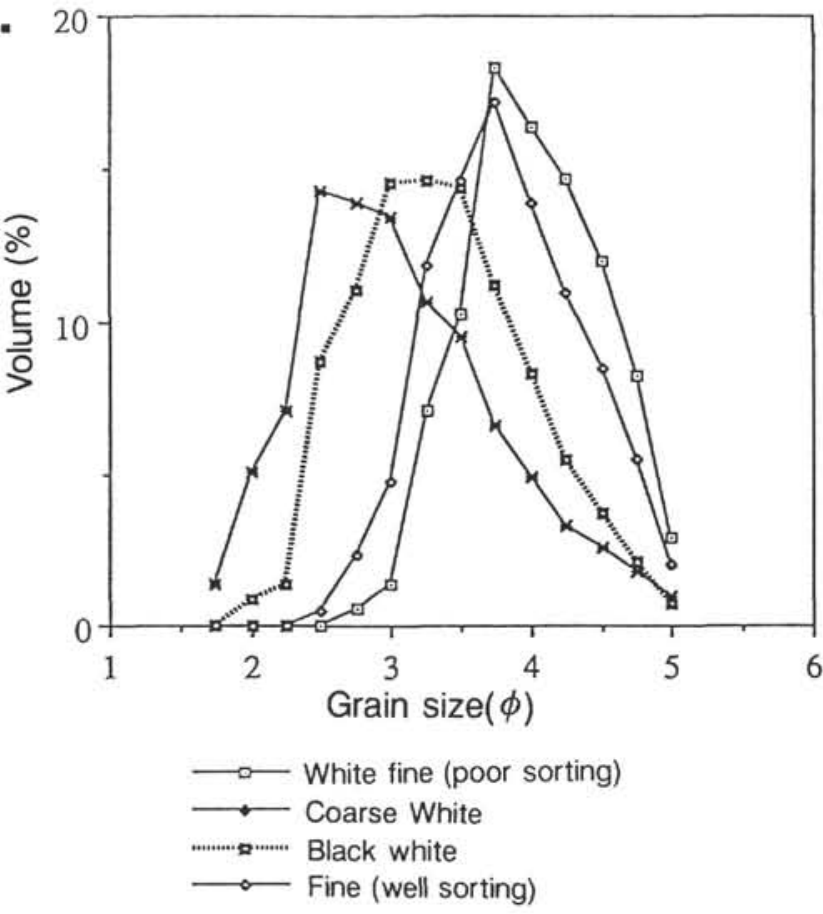

Figure 10. Grain-size characteristics of four types of tephras. A. Cumulative frequency grain-size curve for all the tephras at Site 792, Izu-Bonin forearc. B. Frequency grain-size distribution curve for tephra layers at Site 792. The scale of the grain size is represented by $\phi$ (phi).

this volume). The Pliocene Shimajiri Group of the Ryukyu Arc contains much tephra material. Backarc rifting of the Ryukyu Trough during the Quaternary, characterized by notably bimodal chemistry, has been documented by Letouzey and Kimura (1985).

\section{CONCLUSIONS}

In the Izu-Bonin forearc region, 393 white and black tephras were deposited from the Oligocene to the present. The tephra frequency increased in the late Eocene to early Oligocene and the Pliocene-Pleistocene, with a small increase in the middle Miocene (about $10 \mathrm{Ma}$ ). Quaternary tephras, with variable ratios of basaltic and rhyolitic compositions, increase in frequency at around 0.6 and $0.3 \mathrm{Ma}$. 


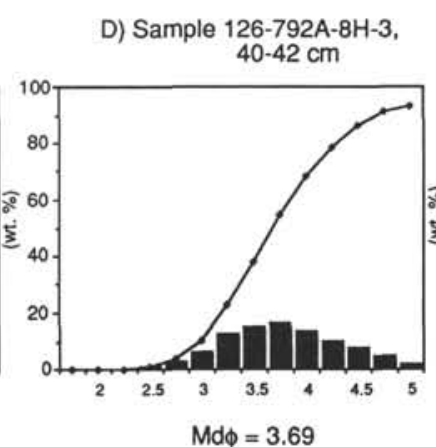

$M d \phi=3.69$

E) Sample 126-792E-3R-1,

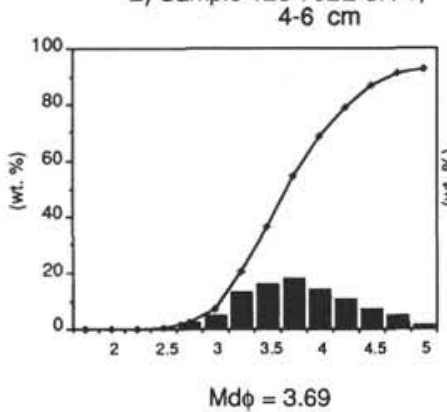

High $\mathrm{K}_{2} \mathrm{O}$

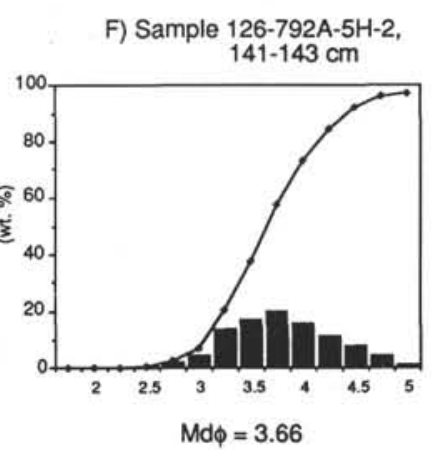

G) Sample 126-792E-18R-1, $108-110 \mathrm{~cm}$

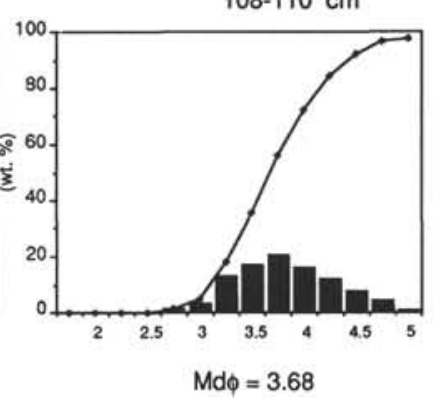

Black

B

Medium $\mathrm{K}_{2} \mathrm{O}$

B) Sample 126-792A-4H-4.

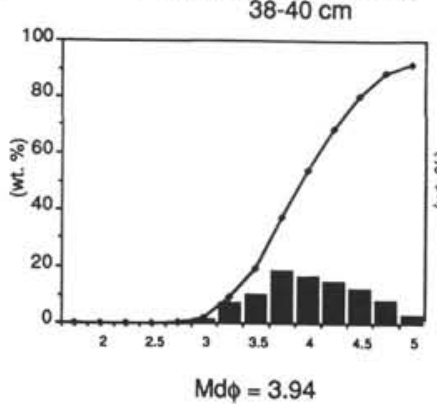
$96-98 \mathrm{~cm}$

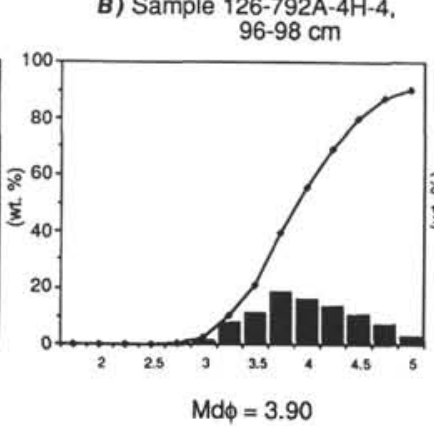

$\mathrm{Md} \phi=3.90$
C) Sample 126-792A-5H-2, $45-47 \mathrm{~cm}$

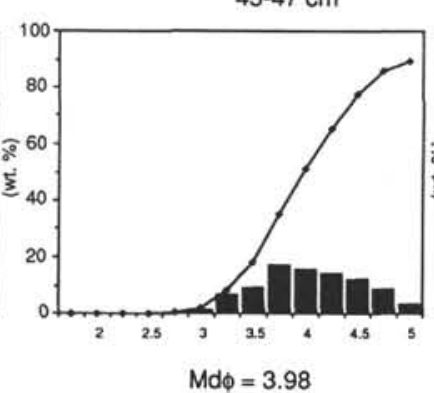

$\mathrm{Md} \phi=3.98$
D) Sample 126-792A-3H-2 $83-85 \mathrm{~cm}$

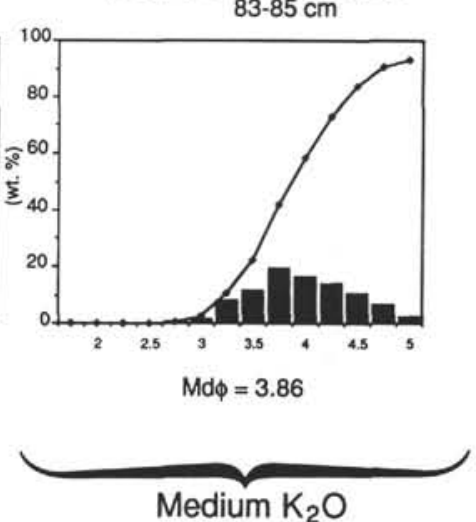

Figure 11. Cumulative curve for grain size of the tephras of the forearc sites of the Izu-Bonin Arc. A. Examples of cumulative curves showing nearly symmetrical grain-size distribution. B. Examples of fine, skewed grain-size distribution.

The Eocene through Quaternary tephras have three principal combinations of color and grain-size distribution: (1) white, coarse, well-sorted, and unskewed; (2) fine, white, well to poorly sorted, generally unskewed but rarely skewed toward the fine end; and (3) black, well-sorted, unskewed, and generally leptokurtic. The first and third combinations may reflect proximal sources in the Izu-Bonin Arc, but some tephra layers of the second type may have been derived from distal sources far to the west (e.g., the Ryukyu Arc).

The chemistry of the tephras is notably bimodal (basaltic and rhyolitic) since the late Pliocene, which suggests that the predominant stress field over that period has been tensional, as in the Cascade region of western North America. Most of the tephras belong to the low-alkali tholeiitic series of Kuno $(1960,1966)$ and the low-K series of Gill (1981). High-K series tephras occurred from 3 Ma to the Holocene, some of which may have been derived from the Ryukyu Arc and other sources far to the west.

\section{ACKNOWLEDGMENTS}

During Leg 126 cruise, Captain Ribbon and the crew of JOIDES Resolution were very helpful to us, sometimes far beyond their official duties, and we are very thankful. We greatly appreciate the many valuable discussions and suggestions of B. Taylor, J. Gill, R. Hiscott, and K. Marsaglia. Thanks are also owed to Drs. F. McCoy, M. Yuasa, S. Nagaoka, N. Kotake, Y. Hirať, H. Matsuoka, and R. U. Solidum for their critical comments. Mrs. Kanehara, Miss K. Ikari, and Ms. Minnie Jones helped us prepare the manuscript.

\section{REFERENCES}

Cadet, J. P., and Fujioka, K., 1980. Neogene volcanic ashes and explosive volcanism: Japan Trench transect, Leg 57, Deep Sea Drilling Project. In von Heune, R., Nasu, N., et al., Initial Repts. DSDP, 56, 57, Pt. 2: Washington (U.S. Govt. Printing Office), 1027-1041. 

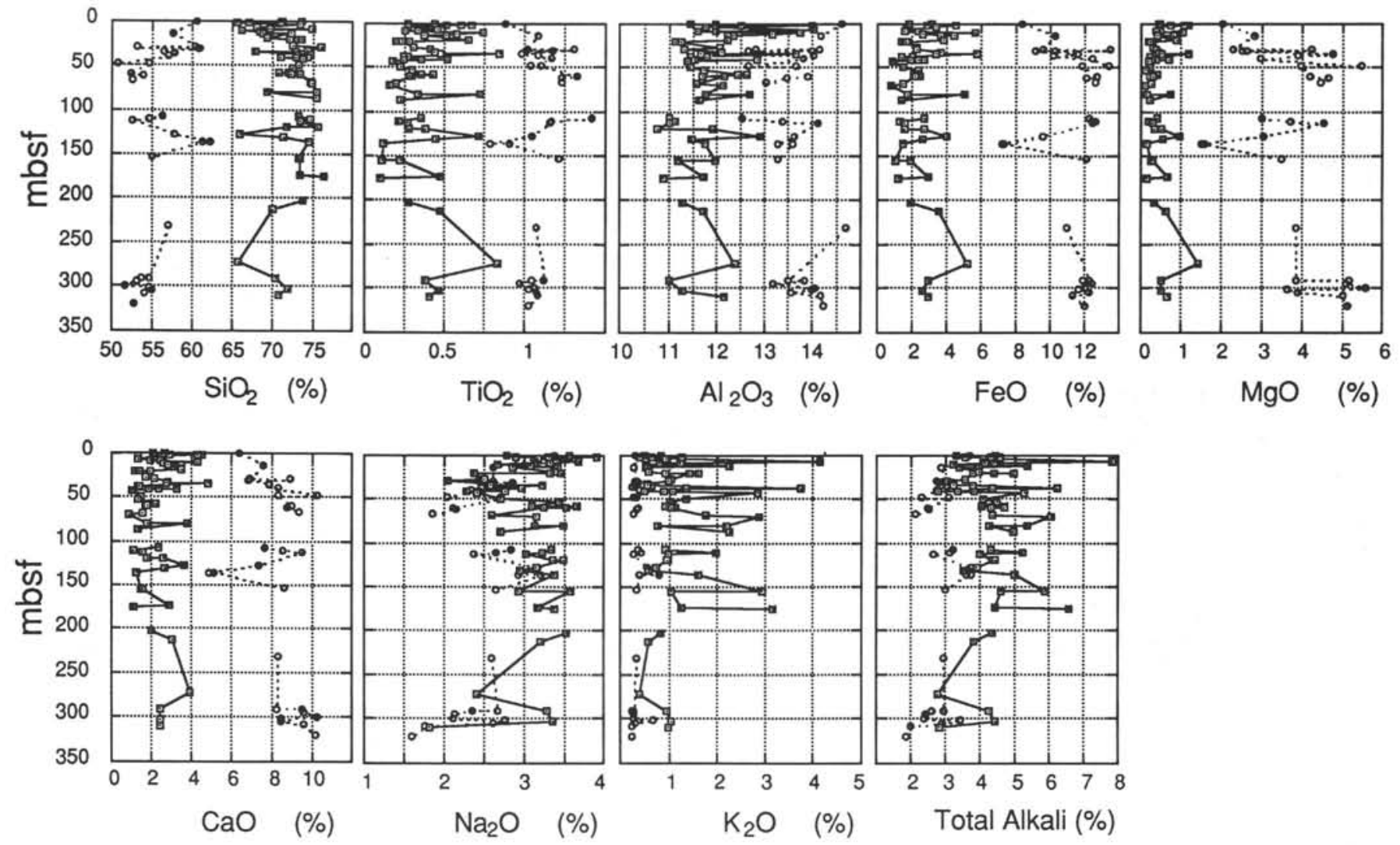

Figure 12. Oxide variation diagram (Harker diagram) of glass shards with time at Site 792 Izu-Bonin forearc. Open circles = black tephras, and open square with dot $=$ white tephras. Horizontal scales are represented by weight percent of oxides.

Christiansen, R. L., and Lipman, P. W., 1972. Cenozoic volcanism and platetectonic evolution of the western United States. II. Late Cenozoic. Philos. Trans. R. Soc. London, Ser. A, 271:249-284.

Crawford, A. J., Beccaluva, L., and Serri, G., 1981. Tectono-magmatic evolution of the West Philippine-Mariana region and the origin of boninites. Earth Planet. Sci. Lett., 54:346-356.

Dickinson, W. R., 1968. Circum-Pacific andesite types. J. Geophys. Res., 73:2261-2269.

Dickinson, W. R., and Hatherton, T., 1967. Andesitic volcanism and seismicity around the Pacific. Science, 157:801-803.

Eaton, G. P., 1982. The Basin and Range Province: origin and tectonic significance. Annu. Rev. Earth Planet. Sci., 10:409-440.

, 1984. The Miocene Great Basin of western North America as an extending back-arc region. Tectonophysics, 102:275-295.

Fryer, P., Pearce, J. A., Stokking, L. B., et al., 1990. Proc. ODP, Init. Repts., 125: College Station TX (Ocean Drilling Program).

Fujioka, K., 1983a. History of the explosive volcanism of the Tohoku Arc from the core sediment samples of the Japan Trench. J. Volcanol. Soc. Jpn., 28:41-58.

_ 1983b. Where were the "Kuroko deposits" formed-looking for the present day analogy. Min. Geol. (Tokyo) Spec. Vol., 11:55-68.

, 1988. A possible nascent rift in northern Izu-Ogasawara arc. Tectonics of Eastern Asia and Western Pacific Continental Margin. 1988 DELP Tokyo Int. Symp. and Sixth Japan-U.S.S.R. Geotectonic Symp., 25. (Abstract)

Fujioka, K., Furuta, T., and Arai, F., 1980. Petrography and geochemistry of volcanic glass: Leg 57, Deep Sea Drilling Project. In von Huene, R., Nasu, N., et al., Init. Repts. DSDP, 56, 57, Pt. 2: Washington (U.S. Govt. Printing Office), 1049-1066.

Furukawa, M., and Isezaki, N., in press. Tectonics of the Okinawa Trough. J. Geol.

Furuta, T., and Arai, F., 1980a. Petrographic and geochemical properties of tephras in Deep Sea Drilling Project cores from the north Philippine Sea.
In Klein, G. deV., Kobayashi, K. et al,, Init. Repts. DSDP, 58: Washington (U.S. Govt. Printing Office), 617-627.

1980b. Petrographic properties of tephras, Leg 56, Deep Sea Drilling Project. In von Huene, R., Nasu, N., et al., Init. Repts. DSDP, 56, 57, Pt. 2: Washington (U.S. Govt. Printing Office), 1043-1048.

Furuta, T., Fujioka, K., and Arai, F., 1986. Widespread submarine tephra around Japan, petrographic and chemical property. Mar. Geol., 72:125-146.

Gill, J. B., 1981. Orogenic Andesites and Plate Tectonics: New York (Springer-Verlag).

Hamuro, K., Aramaki, S., Fujioka, K., Ishii, T., Tanaka, T., and Uto, K., 1983. The Higashi-Izu-oki submarine volcanoes, Part 2, and the submarine volcanoes near the Izu Shoto Islands. Tokyo Daigaku Jishin Kenkyusho Iho, 58:527-557.

Hamuro, K., Aramaki, S., Kagami, H., and Fujioka, K., 1980. The HigashiIzu-oki Submarine Volcanoes, Part 1. Tokyo Daigaku Jishin Kenkyusho Iho, 55:259-297.

Hochstaedter, A. G., and Gill, J. B., 1990. Petrology and geochemistry of lavas from the Sumisu Rift: an incipient back-arc basin. Earth Planet. Sci. Lett., 100:195-209.

Honza, E., and Tamaki, K., 1985. The Bonin Arc. In Nairn, A.E.M., Stehli, F. G., and Uyeda, S. (Eds.), The Ocean Basins and Margins (Vol. 7): The Pacific Ocean: New York (Plenum Press), 459-502.

Honza, E., Tamaki, K., Yuasa, M., Tanahashi, M., and Nishimura, A., 1982. Geological map of the Northern Ogasawara Arc. Geol. Surv. Jpn., Mar. Geol. Map Ser., 17 (Scale 1:1,000,000).

Horn, D. R., Delach, M. N., and Horn, B. N., 1969. Distribution of tephra layers and turbidities in the north Pacific. Geol. Soc. Am. Bull., 80:1715-1724.

Hussong, D. M., and Uyeda, S., 1982. Tectonic processes and the history of the Mariana Arc: a synthesis of the results of Deep Sea Drilling Project Leg 60. In Hussong, D. M., Uyeda, S., et al., Init. Repts. DSDP, 60: Washington (U.S. Govt. Printing Office), 909-929. 

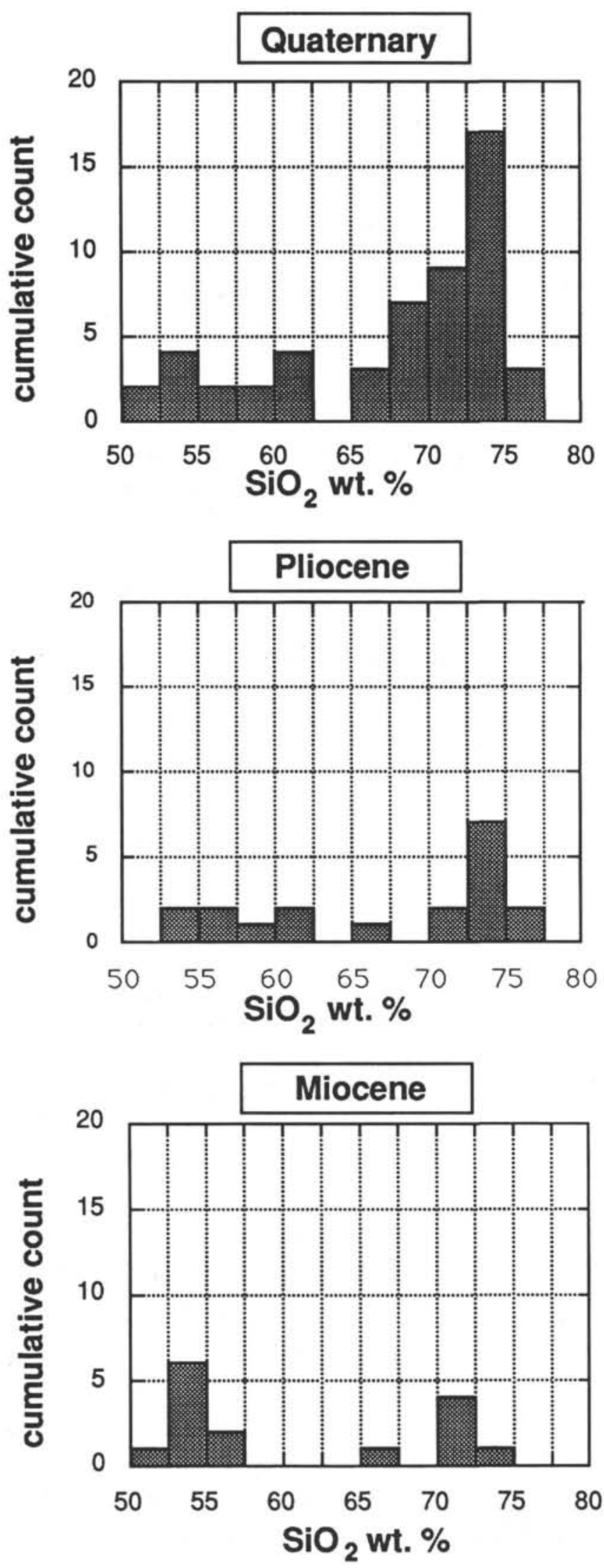

Figure 13. Frequency diagram of $\mathrm{SiO}_{2}$ contents of the tephras examined from the Quaternary, Pliocene, and Miocene.
Ikeda, Y., and Yuasa, M., 1989. Volcanism in nascent back-arc basins behind the Shichito Ridge and adjacent areas in the Izu-Ogasawara arc, northwest Pacific: evidence for mixing between E-type MORB and island arc magmas at the initiation of back-arc rifting. Contrib. Mineral. Petrol., 101:377-393.

Isshiki, N., 1959. Hachijo-jima. Geol. Surv. Jpn., Quadrangle. Ser. (Scale 1:50,000). , 1960. Miyake-jima. Geol. Surv. Jpn., Geol. Map Jpn. (Scale $1: 50,000)$

, 1978. Geology of the Toshima district. Geol. Surv. Jpn., Quadrangle Ser. (Scale 1:50,000).

, 1980. Geology of the Mikurajima, Inambajima and Zenisu district. Geol. Surv. Jpn., Quadrangle Ser. (Scale 1:50,000).

-1982. Geology of the Kozushima district. Geol. Surv. Jpn., Quadrangle Ser. (Scale 1:50,000).

1984. Geology of the O Shima district. Geol. Surv. Jpn, Quadrangle Ser. (Scale 1:50,000).

1987. Geology of the Nii Jima district. With geological sheet map at 1:50,000. Geol. Surv. Jpn.

Karig, D. E., 1971a. Origin and development of marginal basins in the Western Pacific. J. Geophys. Res., 76:2542-2561.

1971b. Structural history of the Mariana Island Arc system. Geol. Soc. Am. Bull., 82:323-344.

1983. Temporal relationships between back-arc basin formation and arc volcanism with special reference to the Philippine Sea. In Hayes, D. E. (Ed.), The Tectonic and Geological Evolution of Southeast Asian Seas and Islands (Pt. 2). Am. Geophys. Union., AGU Monogr. Ser., 27:318-325.

Karig, D. E., and Moore, G. F., 1975a. Tectonic complexities in the Bonin arc system. Tectonophysics, 27:97-118.

1975b. Tectonically controlled sedimentation in marginal basins. Earth Planet. Sci. Lett., 26:233-238.

Karig, D. E., Ingle, J. C., Jr., et al., 1975. Init. Repts. DSDP, 31: Washington (U.S. Govt. Printing Office).

Kennett, J. P., 1981. Marine tephrochronology. In Emiliani, C. (Ed.), The Sea (Vol. 7): The Oceanic Lithosphere: New York (Wiley), 1373-1436.

Kennett, J. P., MacBirney, A. R., and Thunell, R. C., 1979. Episodes of Cenozoic volcanism in Circum-Pacific region. J. Volcanol. Geothermal Res., 2:145-163.

Klein, G. deV., and Kobayashi, K., 1980. Geological summary of the north Philippine Sea, based on Deep Sea Drilling Project Leg 58 results. In Klein, G. deV., Kobayashi, K., et al., Init. Repts. DSDP, 58: Washington (U.S. Govt. Printing Office) 951-962.

Kobayashi, K., and Nakada, M., 1978. Magnetic anomalies and tectonic evolution of the Shikoku inter-arc basin. In Uyeda, S., et al. (Eds.), Geodynamics of the Western Pacific. Adv. Earth Planet. Sci., 7:391-402.

Konda, T., 1974. Bimodal volcanism in the Northeast Japan arc. J. Geol. Soc. Jpn., 80:81-89.

Konishi, K., 1965. Geotectonic framework of the Ryukyu Islands (Nanseishoto). J. Geol. Soc. Jpn., 71:437-457.

Kotake, N., 1988. Upper Cenozoic marine sediments in southern part of the Boso Peninsula, central Japan, J. Geol. Soc. Jpn., 94:187-206.

Kuno, H., 1960. High-alumina basalt. J. Petrol., 1:121-145.

1966. Lateral variation of basalt magma type across continental margins and island arcs. Bull. Volcanol., 19:195-222.

Kurasawa, H., 1959. Petrology and chemistry of the Amagi volcanic rocks, Izu Peninsula, Japan. Chikyu-kagaku, 44:1-18. (In Japanese with English abstract).

Kurasawa, H., and Michino, K., 1976. Petrology and chemistry of the volcanic rocks from the western and southern part of Izu Peninsula, central Japan. J. Volcanol. Soc. Jpn., 21:11-29.

Leg 125 Shipboard Scientific Party, 1989. Plumbing the Pacific sinks. Nature, 339:427-428.

Leg 126 Shipboard Scientific Party, 1989. Arc volcanism and rifting. Nature, 342:18-20.

Letouzey, J., and Kimura, M., 1985, Okinawa trough genesis: structure and evolution of a backarc basin developed in a continent. Mar. Pet. Geol., 2:111-130.

Machida, H., and Arai, F., 1983. Extensive ash falls in and around the sea of Japan from large late Quaternary eruptions. J. Volcanol. Geotherm. Res., 18:151-164.

1988. A review of late Quaternary deep-sea tephras around Japan. Quart. Res., 26:227-242. 
A
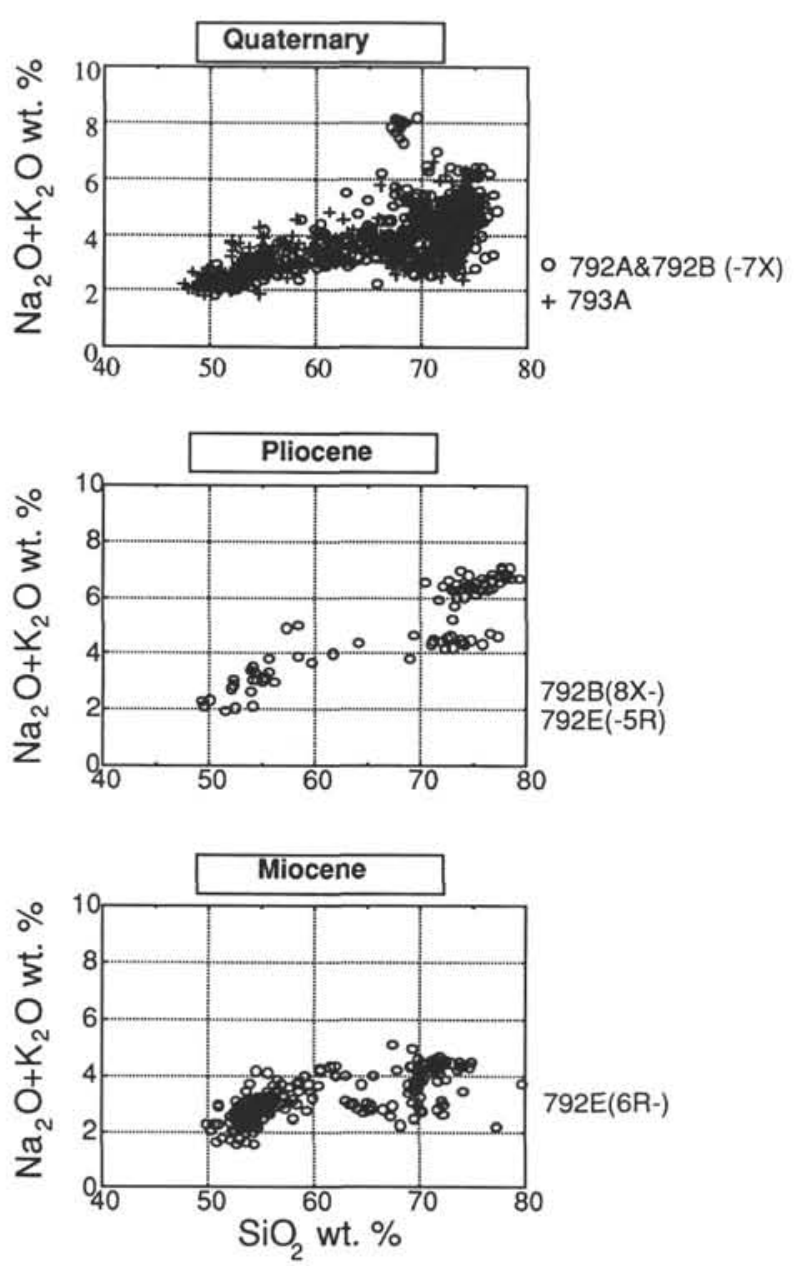

B
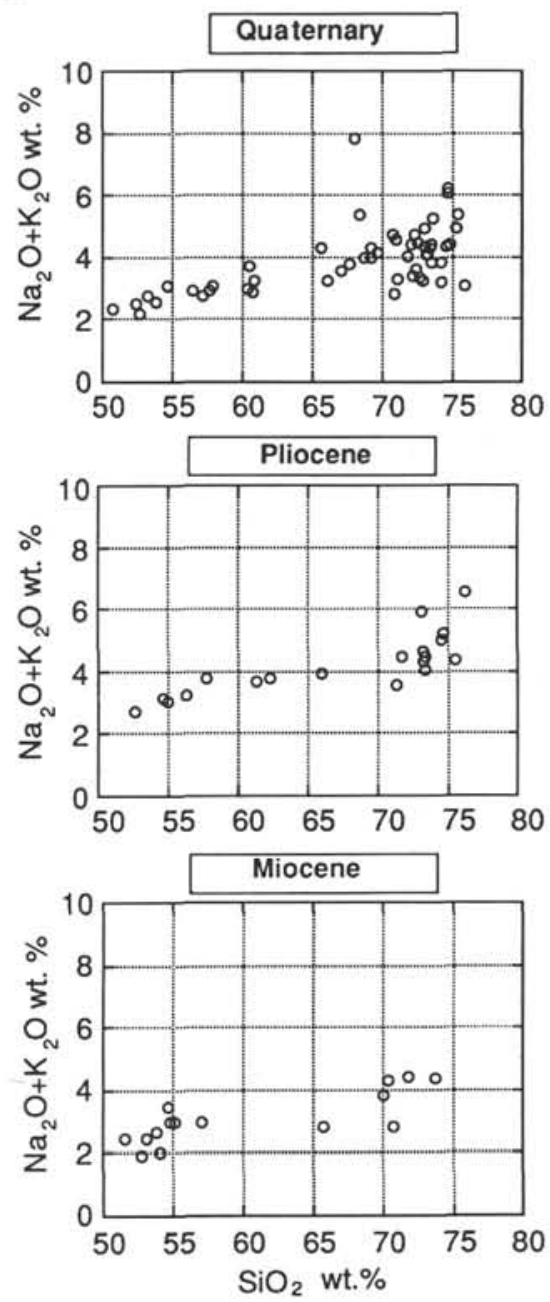

Figure 14. Silica vs. total alkali diagram of the tephras examined at Sites 792 and 793 from the Quaternary, Pliocene, and Miocene. A. Plot of all the data. Open circles $=$ Holes 792A and 792B, and plus signs $=$ Hole 793A. B. Plot of averaged data for selected volcanic tephra layer.

Miyashiro, A., 1973. The Troodos ophiolitic complex was probably formed in an island arc. Earth Planet. Sci. Lett., 19:218-224.

, 1974. Volcanic rock series in island arcs and active continental margins. Am. J. Sci., 274:321-355.

Nakamura, K., 1964. Volcano-stratigraphic study of Oshima Volcano, Izu. Tokyo Daigaku Jishin Kenkyusho Iho, 42:649-728.

Nielson, D. R., and Stoiber, R. E., 1973. Relationship of potassium content in andesitic lavas and depth to the seismic zone. J. Geophys. Res., 78:6887-6892.

Niino, H., Kumagori, T., Tsuya, H., Morimoto, R., Ossaka, G., Hamaguchi, H., Tatsumoto, M., Matsue, Y., Komaki, Y., Arihasa, C., Ebina, K., and Takagi, K., 1953. Report of the submarine eruption of Myojin-Sho. J. Tokyo Univ. Fish., (Spec. Iss.), 40:52.

Notsu, K., Isshiki, N., and Hirano, M., 1983. Comprehensive strontium isotope study of Quaternary volcanic rock from the Izu-Ogasawara arc. Geochem. J., 17:289-302.

Rodolfo, K. S., 1980. Sedimentological summary: clues to arc volcanism, arc sundering, and back-arc spreading in the sedimentary sequences of Deep Sea Drilling Project Leg.59. In Kroenke, L., Scott, R., et al., Init. Repts. DSDP, 59: Washington (U.S. Govt. Printing Office), 621-624.

Scott, R., and Kroenke, L., 1980. Evolution of back-arc spreading and arc volcanism in the Philippine Sea: interpretation of Leg 59 DSDP results. In Hayes, D. E. (Ed.), The Tectonic and Geologic Evolution of Southeast Asian Seas and Islands. Am. Geophys. Union, Geophys. Monogr. Ser., 23:283-292.
Scott, R. B., Kroenke, L., Zakariadze, G., and Sharaskin, A., 1980. Evolution of the south Philippine Sea: DSDP Leg 59 results. In Kroenke, L., Scott, R., et al., Init. Repts. DSDP, 59: Washington (U.S. Govt. Printing Office), 803-816.

Seno, T., and Maruyama, S., 1984. Paleogeographic reconstruction and origin of the Philippine Sea. Tectonophysics, 102:53-84.

Sharaskin, A. Y., Bogdanov, N. A., and Zakariadze, G. S., 1981. Geochemistry and timing of the marginal basin and arc magmatism in the Philippine Sea. Philos. Trans. R. Soc. London, Ser. A, 300:287-297.

Shiraki, K., Kuroda, N., and Maruyama, S., 1978. Evolution of the Tertiary volcanic rocks in the Izu-Mariana Arc. Bull. Volcanol., 41:548-562.

Smith, D.G.W., and Westgate, J. A., 1969. Electron probe technique for characterizing pyroclastic deposits. Earth Planet. Sci. Lett., 5:313-319.

Sparks, R.S.J., Wilson, L., and Sigurdsson, H., 1981. The pyroclastic deposits of the 1875 eruption of Askja, Iceland. Philos. Trans. R. Soc. London, Ser. A, 299:241-273.

Suga, K., and Fujioka, K., 1990. Volume of volcanic materials along northern Izu-Bonin Arc. J. Volcanol. Soc. Jpn., 35:359-374.

Takada, A., Oshima, O., Aramaki, S., Ono, K., Yoshida, T., and Kajima, K., in press. Geology of Aogashima Volcano, Izu Islands, Japan. Bull. Volcanol. Soc. Jpn.

Taylor, B., Fujioka, K., et al., 1990. Proc. ODP, Init. Repts., 126: College Station, TX (Ocean Drilling Program).

Taylor, R. N., and Nesbitt, R. W., 1990. Geology and geochemistry of the Izu-Bonin fore-arc region: results from ODP Leg 126 and the Bonin 
A
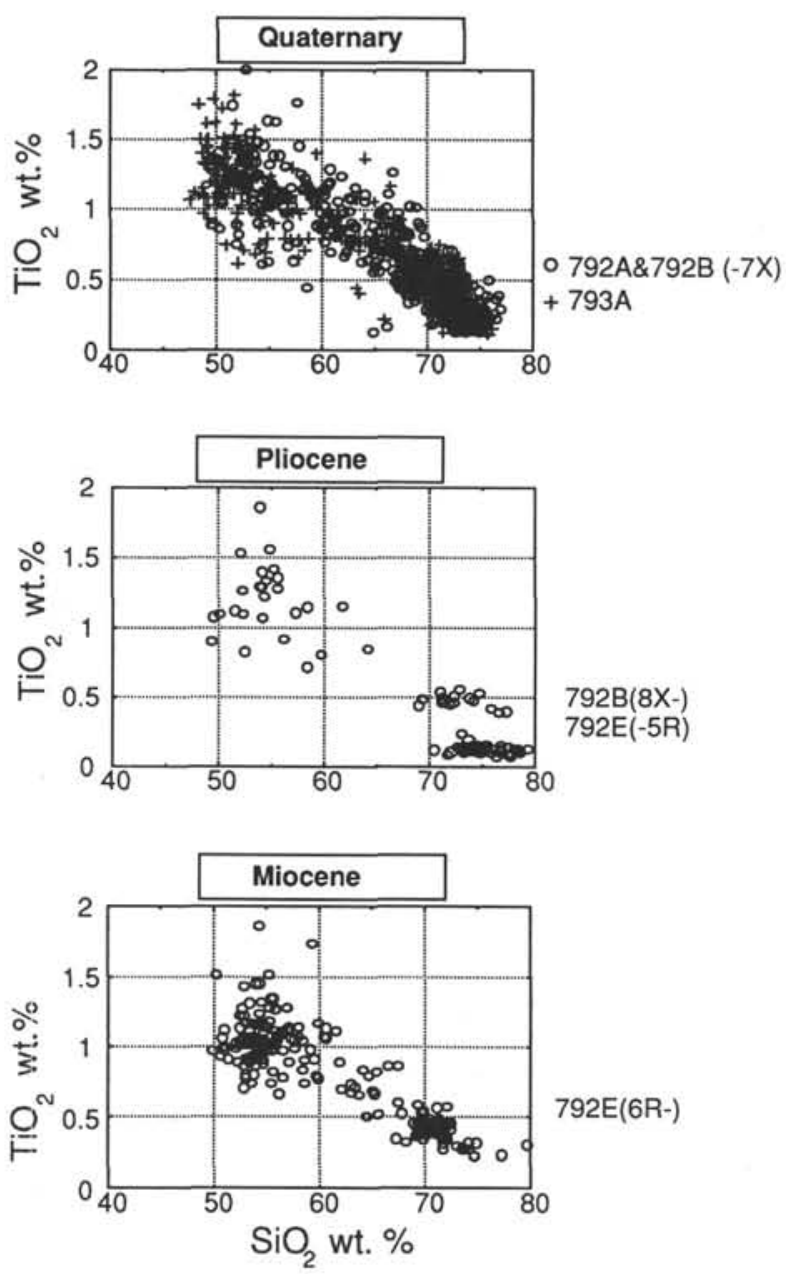

B
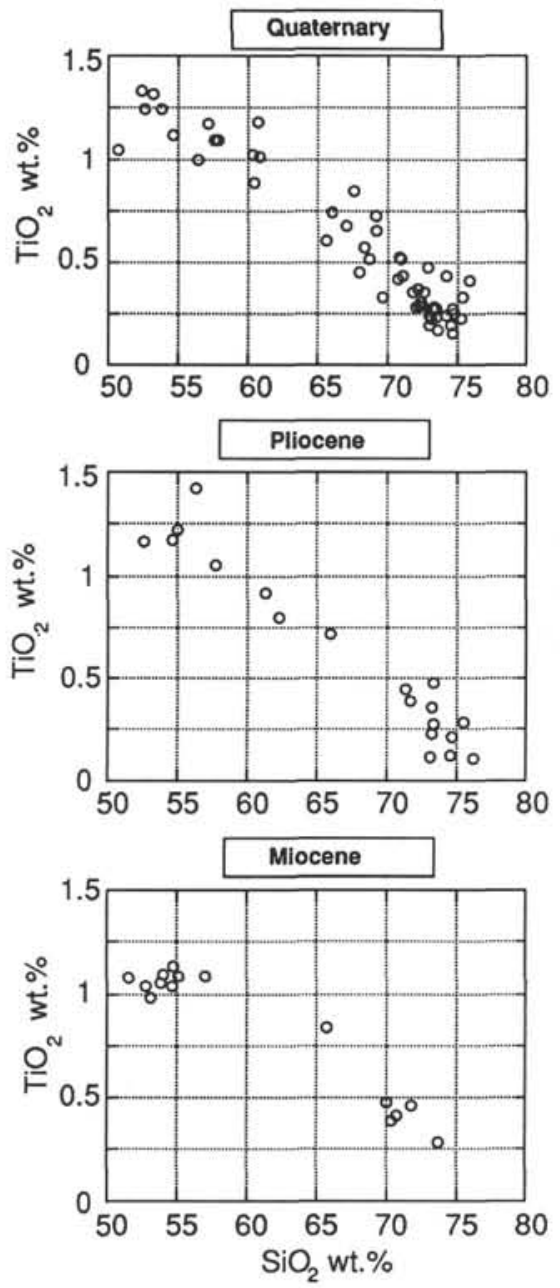

Figure 15. $\mathrm{SiO}_{2}$ vs. $\mathrm{TiO}_{2}$ diagram for tephras of the forearc Sites 792 and 793, Izu-Bonin Arc, from the Quaternary, Pliocene, and Miocene. A. Plot of all the data. Open circles $=$ Holes 792A and 792B, and plus signs $=$ Hole 793A. B. Plot of averaged data for selected volcanic tephra layer.

Islands. Fifth Circum-Pacific Energy and Mineral Resources Conference and Exhibition, Honolulu, Hawaii. (Abstract)

Tsuya, H., 1937. On the volcanism of the Huzi Volcanic Zone, with special reference to the geology and petrology of Izu and the Southern Islands. Tokyo Daigaku Jishin Kenkyusho Iho, 15:215-357.

Walker, G.P.L., 1971. Grain size characteristics of pyroclastic deposits. J. Geol., 79:696-714.

Yokoyama, T., Danhara, T., and Yamashita, T., 1986. A new refractometer for volcanic glass. J. Volcanol. Soc. Jpn., 25:21-30.

Yuasa, M., 1985. Sofugan Tectonic Line, a new tectonic boundary separating northern and southern parts of the Ogasawara (Bonin) arc, northwest
Pacific. In Nasu, N., Kobayashi, K., Uyeda, S., Kushiro, I., and Kagami, H. (Eds.), Formation of Active Ocean Margins: Tokyo (Terra Scientific), 483-496.

Yuasa, M., and Murakami F., 1985. Geology and geomorphology of the Ogasawara Arc and the Sofugan Tectonic Line. J. Geogr., 94:47-66.

Date of initial receipt: 18 March 1991

Date of acceptance: 2 August 1991

Ms 126B-117 
A
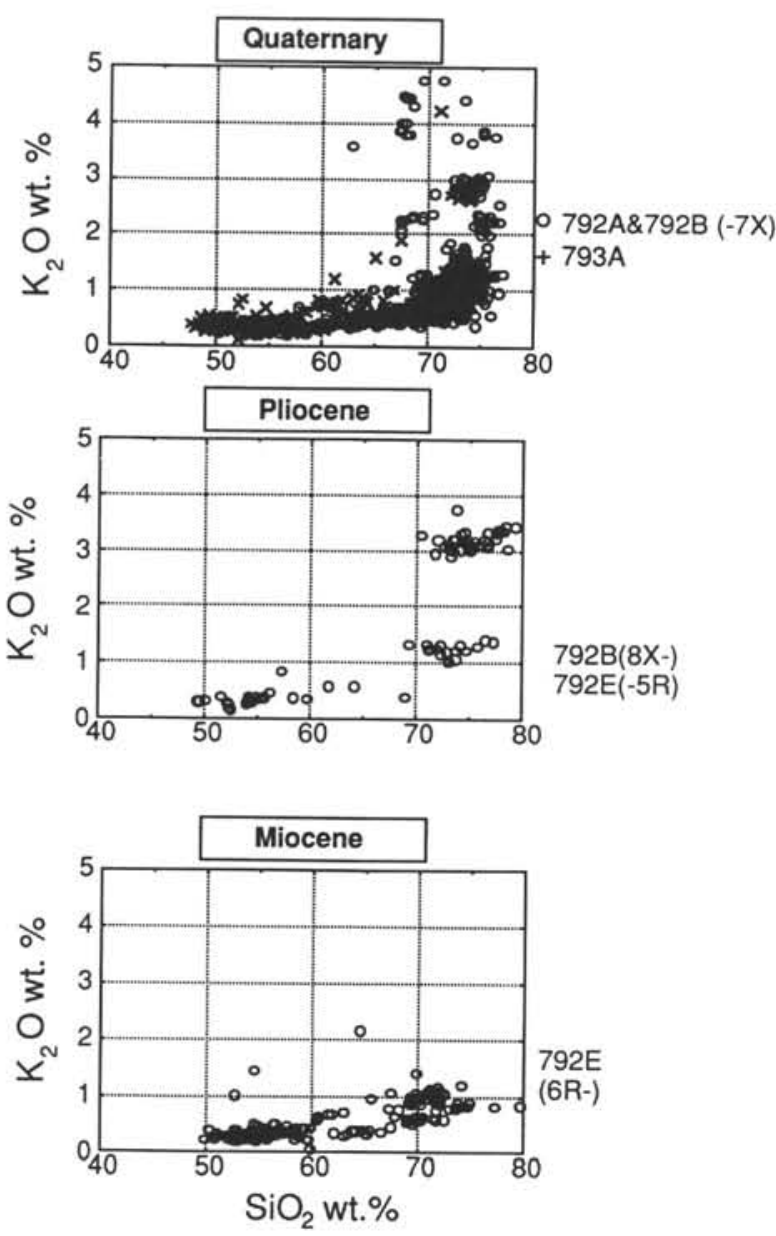

B
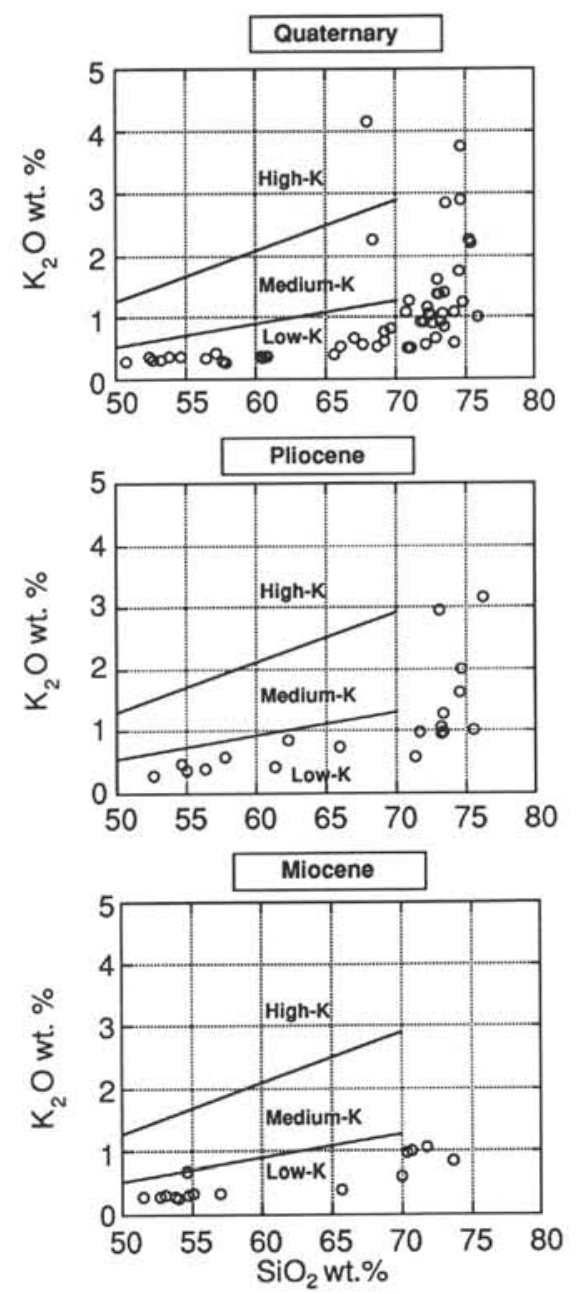

Figure 16. $\mathrm{SiO}_{2}$ vs. $\mathrm{K}_{2} \mathrm{O}$ diagram for tephras of the forearc Sites 792 and 793, Izu-Bonin Arc, from the Quaternary, Pliocene, and Miocene. Categories low-k to high-K are the same as in Gill (1981). A. Plot of all the data. Open circle $=$ Holes 792A and 792B, and cross $=$ Hole 793A. B. Plot of averaged data for selected volcanic tephra layer. 